\title{
Enhanced Light Extraction Efficiency from GaN Light Emitting Diodes using Photonic Crystal Grating Structures
}

\author{
A Thesis Presented to the \\ Electrical Engineering Department Faculty of \\ California Polytechnic State University, San Luis Obispo
}

\author{
In Partial Fulfillment \\ of the Requirements for the Degree \\ Master of Science in Electrical Engineering
}

By

Simeon Su-Ming Trieu

June 2010 
(C) 2010

Simeon S. Trieu

\section{ALL RIGHTS RESERVED}

- ii - 


\section{COMMITTEE MEMBERSHIP}

Title: Enhanced Light Extraction Efficiency from GaN Light Emitting Diodes using Photonic Crystal Grating Structures

Author: $\quad$ Simeon Su-Ming Trieu

Date Submitted: June 10,2010

Dr. Xiaomin Jin, Assistant Professor

Committee Chair

Dr. Dennis Derickson, Department Chair

Committee Member

Dr. David Braun, Professor

Committee Member 


\begin{abstract}
Title: Enhanced Light Extraction Efficiency from GaN Light Emitting Diodes using Photonic Crystal Grating Structures

Author: Simeon Su-Ming Trieu
\end{abstract}

Gallium nitride (GaN) light emitting diodes (LED) embody a large field of research that aims to replace inefficient, conventional light sources with LEDs that have lower power, higher luminosity, and longer lifetime. This thesis presents an international collaboration effort between the State Key Laboratory for Mesoscopic Physics in Peking University (PKU) of Beijing, China and the Electrical Engineering Department of California Polytechnic State University, San Luis Obispo. Over the course of 2 years, Cal Poly's side has simulated GaN LEDs within the pure blue wavelength spectrum $(460 \mathrm{~nm})$, focusing specifically on the effects of reflection gratings, transmission gratings, top and bottom gratings, error gratings, 3-fold symmetric photonic crystal, and 2-fold symmetric nano-imprinted gratings. PKU used our simulation results to fabricate GaN high brightness LEDs from the results of our simulation models. We employed the use of the finite difference time domain (FDTD) method, a computational electromagnetic solution to Maxwell's equations, to measure light extraction efficiency improvements of the various grating structures. Since the FDTD method was based on the differential form of Maxwell's equations, it arbitrarily simulated complex grating structures of varying shapes and sizes, as well as the reflection, diffraction, and dispersion of propagating light throughout the device. 
We presented the optimized case, as well as the optimization trend for each of the single grating structures within a range of simulation parameters on the micron scale and find that single grating structures, on average, doubled the light extraction efficiency of GaN LEDs. Photonic crystal grating research in the micron scale suggested that transmission gratings benefit most when grating cells tightly pack together, while reflection gratings benefit when grating cells space further apart. The total number of grating cells fabricated on a reflection grating layer still affects light extraction efficiency. For the top and bottom grating structures, we performed a partial optimization of the grating sets formed from the optimized single grating cases and found that the direct pairing of optimized single grating structures decreases overall light extraction efficiency. However, through a partial optimization procedure, top and bottom grating designs could improve light extraction efficiency by $118 \%$ for that particular case, outperforming either of the single top or bottom grating cases alone. Our research then explored the effects of periodic, positional perturbation in grating designs and found that at a $10-15 \%$ randomization factor, light extraction efficiency could improve up to $230 \%$ from the original top and bottom grating case. Next, in an experiment with PKU, we mounted a 2fold symmetric photonic crystal onto a PDMS hemi-cylinder by nano-imprinting to measure the transmission of light at angles from near tangential to normal. Overall transmission of light compared with the non-grating design increases overall light extraction efficiency when integrated over the range of angles. Finally, our research focused on the 3-fold symmetric photonic crystal grating structure and employed the use of 3-D FDTD methods and incoherent light sources to better study the effects of higher- 
ordered symmetry in grating design. Grating cells were discovered as the source of escaping light from the GaN LED model. The model revealed that light extraction efficiency and the far-field diffraction pattern could be estimated by the position of grating cells in the grating design. 


\section{ACKNOWLEDGEMENTS}

First and foremost, I must thank my thesis advisor, Prof. Xiaomin Jin. Without her help and constant guidance throughout my academic journey, I would never have achieved an NSF fellowship, published a journal paper, won a research competition, or presented at a conference. Prof. Jin has been my supporter and took a personal interest in my education, both in academia and in life.

I also thank all of my family and friends who have put up with my anti-social behavior for the past 3 years. You still come out to the conference presentations, award ceremonies, and research competitions to support me. It really means a lot to me.

This project is supported by the Department of the Navy, Office of Naval Research, under Award \# ONR 6-N00014-07-1-1152 in 2008, Award \# ONR 7-N000140811209 in 2009.

The NSF 08-603 OISE - EAPSI 2009 fellowship award ("EAPSI: Light Extraction Improvement of GaN-based Light Emitting Diodes") in summer 2009 supported the 3fold symmetric photonic crystals on GaN flip-chip LEDs, as well as, transmitted diffraction simulations and experiments.

I would like to thank Prof. Bei Zhang, Dr. Xiang Ning Kang, and Dr. Guo Yi Zhang from Peking University for providing expert advice on the GaN LED grating experiments and 
modeling. Thank you for hosting me in Summer 2009 and for giving me the opportunity to put a warm face on the impersonal names of the researchers I sent many e-mails to throughout this research. To all the researchers at Peking University, Xiong Chang, Wei Wei, Sun Yong Jian, and Fu Xing Xing, my stay in China was absolutely wonderful thanks to you. The modest students at PKU were talented and deserved more credit for their work than they would care to admit.

Finally, I would like to thank each of my thesis committee members, Dr. Jin, Dr. Derickson, and Dr. Braun for being patient with my education for all of these three years in the MSEE/MBA program. Thank you for teaching an otherwise unremarkable student how to accomplish remarkable things. 


\section{TABLE OF CONTENTS}

Page

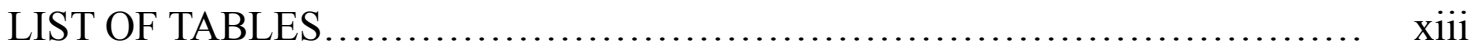

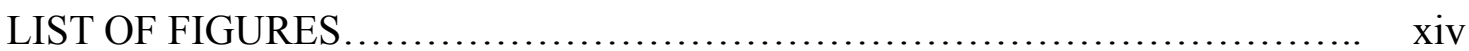

Chapter 1 - Introduction............................................ 1

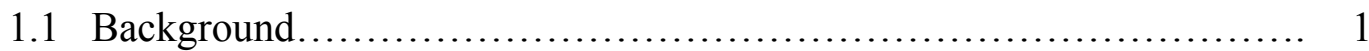

1.2 International Collaboration with Peking University................... 8

Chapter 2 - The Finite Difference Time Domain Method.................... 10

2.1 Derivation of FDTD Theory................................... 10

2.2 Accuracy and Stability of the FDTD Method......................... 14

2.3 Computational Requirements of FDTD.......................... 15

2.4 RSoft Photonics Suite ${ }^{\mathrm{TM}}$ and the FullWAVETM Module................ 16

2.4.1 Construction of Thin-film LEDs with the FullWAVETM Module..... 17

2.4.2 Grid Sizes for Accuracy and Stability in RSoft FullWAVETM ....... 19

2.4.3 Constant Wave Sources in RSoft FullWAVE ${ }^{\mathrm{TM}} \ldots \ldots \ldots \ldots \ldots \ldots \ldots .21$

2.4.4 Simulation Domain and the Perfectly Matched Layer............. 23

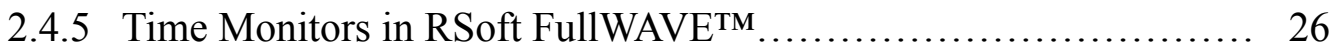

2.4.6 Stop, Monitor, and Update Times in RSoft FullWAVETM ........... 27

2.5 Rsoft_tools Supplemental Software.............................. 28

Chapter 3 - Single Reflection Grating GaN LED Simulations.................. 29

3.1 Single Reflection Grating GaN LED Model........................... 30 
3.2 Single Reflection Grating GaN LED Simulation Results................. 32

3.3 Single Reflection Grating GaN LED Results Summary and Conclusions... 36

Chapter 4 - Top Transmission Grating GaN LED Simulations..................... 38

4.1 Top Transmission Grating Background............................... 38

4.2 Single Top Transmission Grating GaN LED Simulation Results............. 39

4.2.1 Non-Lossy p-GaN Transmission Grating Simulation Results.......... 40

4.2.2 Lossy ITO Transmission Grating Simulation Results................. 44

4.2.3 Non-Lossy ITO Transmission Grating Simulation Results............ 47

4.3 Single Transmission Grating Results Summary and Conclusions............ 50

Chapter 5 - Top and Bottom Grating GaN LED Simulations..................... 52

5.1 Top and Bottom Grating Background............................... 52

5.2 Top and Bottom Grating GaN LED Simulation Results................... 55

5.3 Top and Bottom Grating GaN LED Results Summary and Conclusions..... 63 Chapter 6 - Error Grating GaN LED Simulations................................ 64

6.1 Error Grating GaN LED Model...................................... 64

6.2 Error Grating GaN LED Simulation Results............................ 65

6.3 Error Grating GaN LED Simulation Conclusions....................... 68

Chapter 7 -2-fold Symmetric Transmitted Diffraction Experiments................ 69

7.1 Transmitted Diffraction Experiments and Simulation Results............. 70

7.2 Transmitted Diffraction Experiments and Simulation Conclusions.......... 72 Chapter 8 - 3-fold Symmetric Photonic Crystals and Flip-Chip Based Designs...... 73

8.1 3-fold Symmetric Photonic Crystal Grating and GaN LED Models........ 74 
8.2 3-fold Symmetric Photonic Crystal GaN LED Simulation Results......... 76

8.3 3-Fold Symmetric Photonic Crystal Results Summary and Conclusions.... 80 Chapter 9 - Overall Conclusions and Future Work........................ 81

9.1 Overall Conclusions for Each Grating Type......................... 81

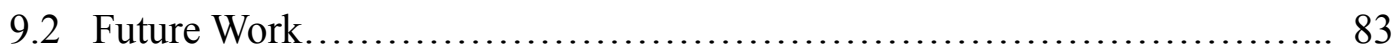

9.2.1 12-fold Symmetric Photonic Quasicrystals..................... 83

9.2.2 Nano-scale Grating Structures............................. 84

9.2.3 Nano-dot Bump Defects................................. 85

9.2.4 Genetic Mutation Algorithms to Determine Absolute Maxima....... 85 APPENDIX A: SUMMARY OF GRATING SIMULATIONS BY RANK......... 87 APPENDIX B: RSOFT_TOOLS SOURCE CODE $\ldots \ldots \ldots \ldots \ldots \ldots \ldots \ldots \ldots \ldots \ldots . \ldots . \ldots \ldots$

PUBLISHED WORKS ............................................... 107

REFERENCES................................................... 109 


\section{LIST OF TABLES}

Table

Page

2.1 Segment Properties with Descriptions............................ 18

$2.2 \quad 3-\mathrm{D}$ Structures Types............................................. 19

3.1 GaN LED Model Simulation Parameters........................... 32

5.1 Summary of Optimized Single Grating Structures..................... 54

5.2 Top and Bottom Grating Simulation Results........................... 55

8.1 Full GaN Model 3-D FDTD Simulations for Best Case Gratings........... 77 


\section{LIST OF FIGURES}

$\begin{array}{lll}\text { Figure } & \text { Page }\end{array}$

$1.1 \quad$ Philips $^{\mathrm{TM}}$ LED Light Bulb.......................................... 1

1.2 Total Internal Reflection Model: (a) cone of transmitted angles and total internal reflection, (b) LED with enhanced cone of transmitted angles, due to affixed resin dome, and (c) incident beam scattering via Bragg diffraction

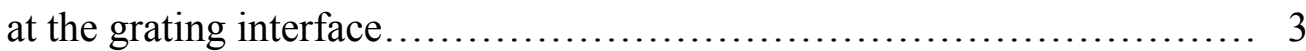

$1.3 \quad$ Absorption within the LED ......................................... 4

1.4 International Collaboration Agreement with Peking University and Cal Poly................................................................ 8

$2.1 \quad$ Yee's Mesh........................................................... 12

2.2 RSoft CAD Environment Window..................................... 17

2.3 Segment Properties Window............................................. 19

$2.4 \quad$ Simulation Parameters Window....................................... 21

2.5 Approximated Plane Wave: (a) emitted from the multiple quantum well region (b) band gap diagram for the $\mathrm{GaN} / \mathrm{InGaN}$ heterojunction [41].......... 22

2.6 Global Default Settings Window..................................... 23

$2.7 \quad$ Launch Parameters Window.............................................. 24

2.8 The PML in Reference to the Simulation Domain.......................... 25

$2.9 \quad$ Time Monitor Window................................................ 27

3.1 Reflection Grating GaN LED Model..................................... 29

3.2 Reflection Grating Structures: (a) cylindrical and (b) conical............... 30 
3.3 Average power for the conical reflection grating case: (a) $\mathrm{A}=1$, (b) $\mathrm{A}=2$,

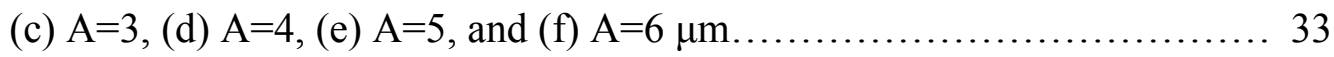

3.4 Average power for the cylindrical reflection grating case: (a) $\mathrm{A}=1$, (b) $\mathrm{A}=2$,

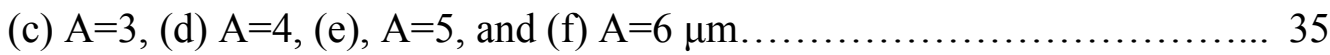

3.5 Peak Power vs. Grating Width for (a) conical and (b) cylindrical reflection

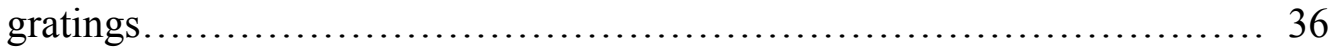

4.1 Transmission Grating GaN LED Model................................... 38

4.2 Transmission Grating Types: (a) conical and (b) cylindrical................ 39

4.3 Average power for the conical non-lossy $\mathrm{p}-\mathrm{GaN}$ transmission grating case:

(a) $\mathrm{A}=1$, (b) $\mathrm{A}=2$, (c) $\mathrm{A}=3$, (d) $\mathrm{A}=4$, (e) $\mathrm{A}=5$, and (f) $\mathrm{A}=6 \mu \mathrm{m} \ldots \ldots \ldots \ldots \ldots$

4.4 Average power for the cylindrical non-lossy $\mathrm{p}-\mathrm{GaN}$ transmission grating

case: (a) $\mathrm{A}=1$, (b) $\mathrm{A}=2$, (c) $\mathrm{A}=3$, (d) $\mathrm{A}=4$, (e) $\mathrm{A}=5$, and (f) $\mathrm{A}=6 \mu \mathrm{m}$.

4.5 Peak Power vs. Grating Width for (a) conical and (b) cylindrical non-lossy

p-GaN transmission gratings.

4.6 Average power for the lossy conical ITO transmission grating case:

(a) $\mathrm{A}=1$, (b) $\mathrm{A}=2$, (c) $\mathrm{A}=3$, (d) $\mathrm{A}=4$, (e) $\mathrm{A}=5$, and (f) $\mathrm{A}=6 \mu \mathrm{m}$.

4.7 Average power for the lossy cylindrical ITO transmission grating case:

(a) $\mathrm{A}=1$, (b) $\mathrm{A}=2$, (c) $\mathrm{A}=3$, (d) $\mathrm{A}=4$, (e) $\mathrm{A}=5$, and (f) $\mathrm{A}=6 \mu \mathrm{m}$.

4.8 Average power for the non-lossy conical ITO transmission grating case:

(a) $\mathrm{A}=1$, (b) $\mathrm{A}=2$, (c) $\mathrm{A}=3$, (d) $\mathrm{A}=4$, (e) $\mathrm{A}=5$, and (f) $\mathrm{A}=6 \mu \mathrm{m}$

4.9 Average power for the non-lossy cylindrical ITO transmission grating case:

(a) $\mathrm{A}=1$, (b) $\mathrm{A}=2$, (c) $\mathrm{A}=3$, (d) $\mathrm{A}=4$, (e) $\mathrm{A}=5$, and (f) $\mathrm{A}=6 \mu \mathrm{m}$. 
4.10 Peak power vs. grating width for non-lossy ITO transmission gratings:

(a) conical and (b) cylindrical..................................... 50

$5.1 \quad$ Top and Bottom Grating GaN LED Model.......................... 52

5.2 Partial Optimization Results: (a) comparison of gratings with only top, only bottom, and double gratings based on Table 5.2 Case 9 (Both top and bottom grating $A=1 \mathrm{~mm}, w=1 \mathrm{~mm}$ ), (b) double grating optimization 1 based on Table 5.2, Case 1, sweeping either bottom grating $\mathrm{d}$ or top grating $\mathrm{d}$, (c) double grating optimization 2 based on Table 5.2, Case 1, but with the top grating $\mathrm{A}=\mathrm{w}=4 \mu \mathrm{m}, \mathrm{d}=52 \mathrm{~nm}$ and bottom grating $\mathrm{A}=4 \mu \mathrm{m}$, while sweeping the $\mathrm{w}$ and $\mathrm{d}$ parameters

5.3 Mode profile of the following grating structures: (a) Top conical grating only $A=4 \mu \mathrm{m}, w=4 \mu \mathrm{m}, \mathrm{d}=164 \mathrm{~nm}$, (b) Bottom cylindrical grating only $A=2 \mu \mathrm{m}$, $\mathrm{w}=1 \mu \mathrm{m}, \mathrm{d}=136 \mathrm{~nm},(\mathrm{c})$ Top and bottom grating (a)+(b), Table 5.2 Case 1. (d) Best top grating: conical $\mathrm{A}=1 \mu \mathrm{m}, \mathrm{w}=1 \mu \mathrm{m}, \mathrm{d}=138 \mathrm{~nm}$ (Table 5.1), (e) Best bottom grating: conical $\mathrm{A}=1 \mu \mathrm{m}, \mathrm{w}=1 \mu \mathrm{m}, \mathrm{d}=200 \mathrm{~nm}$ (Table 5.1), and (f)Non-grating LED case... 61

6.1 Fig. 6.1 - Error grating model: (a) normal reference grating model and (b) error grating model with both. 64

6.2 Results of error grating simulation for (a) conical reflection gratings paired with transmission gratings: case No. 12 , case No. 8, case No. 11, case No. 7, case No. 10, and case No. 9 in Table 5.2. (b) cylindrical grating paired with transmission grating: case No. 6, case No. 1, case No. 5, case No. 3, case 
No. 4, and case No. 2. in Table 5.2 ............................... 66

7.1 2PC Diffraction Experiment Setup: (a) Experimental setup for measuring transmitted diffraction of a 2PC structure mounted on PDMS and (b) 2PC grating structure

7.2 FDTD Simulation Results for the 2PC Transmitted Diffraction Experiment:

(a) Transmitted Diffraction Efficiency simulated by FDTD simulation and

(b) Transmitted Diffraction Efficiency for a non-uniform gridded FDTD

simulation

8.1 Comparison of (a) 2-fold symmetric photonic crystal gratings and

(b) 3-fold symmetric photonic crystal gratings...................... 72

8.2 3-Fold Symmetric Photonic Crystal GaN LED Model.................... 76

8.3 Total output power for a GaN-to-Air grating structure: (a) $A=1 \mu \mathrm{m}$,

(b) $\mathrm{A}=3 \mu \mathrm{m}$, and (c) $\mathrm{A}=6 \mu \mathrm{m}$

8.4 Simulation Domain from a 3-fold Symmetric Photonic Crystal Grating Simulation: (a) xy-plane cut, (b) xz-plane cut....................... 79

9.1 12-fold Symmetric Photonic Quasicrystals: (a) hexagonal packed geometry, (b) circular packed geometry. 


\section{Chapter 1 - Introduction}

\subsection{Background}

The widespread use of the light emitting diode (LED) in commercial applications has driven an intensive field of research that spans electromagnetics, optics, and device physics. The goals of such research aim to develop brighter, cheaper, and more energy efficient light sources to meet the market demand. Fiber optic systems, light bulbs, display technology, and machine vision all make use of LEDs. For example, Fig. 1.1 shows a first generation light bulb created by Philips that contains many LED luminaires, complex PWM circuitry, light diffusers and heat sinks to allow natural-looking light bulbs, that are equivalent to an $80 \mathrm{~W}$ incandescent light bulb, to retrofit into standard Atype sockets.

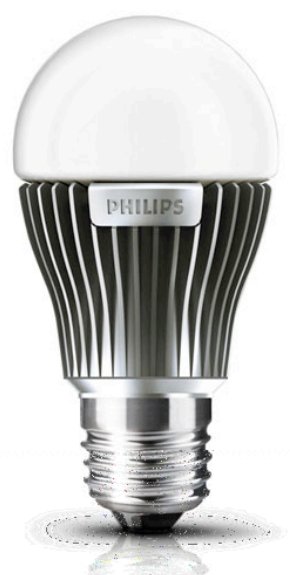

Fig. 1.1 - Philips ${ }^{\mathrm{TM}}$ LED Light Bulb

LEDs must minimize power consumption, generate minimal heat, and maximize extracted lumens per watt, and so, they meet the goals required by the aforementioned practical applications. Currently, two effective LED optimization methods improve the 
performance characteristics of an LED: increasing electroluminescent efficiency and increasing light extraction efficiency [1]. Electroluminescent efficiency measures how efficiently electrons are converted into photons of light, while light extraction efficiency measures how efficiently those generated photons are extracted from the LED (ie. without absorption). This thesis seeks to increase LED efficiency. Therefore, light extraction efficiency of Gallium Nitride (GaN) LEDs must be improved to meet the demanding requirements. Three significant factors contribute to the key inefficiencies in GaN LEDs: 1) GaN's high refractive index, and therefore low critical angle at the semiconductor-air interface, 2) the absorption of light within the device at the multiple quantum well (MQW) region, as well as dislocations and defects in the GaN crystal, and 3) inefficient device geometry [2-4].

A light emissive device's first key limitation results from the device layer's low critical angle that traps generated light internally. In other words, much of the light generated within the devices is totally internally reflected back within the device rather than escaping as emitted light. Fig. 1.2(a) shows the typical problem of a GaN LED with such a high refractive index. The light blue photon coming in at a high angle above the critical angle reflects internally, while the dark blue photon coming in at a low angle below the critical transmits to the air. If the critical angle is sufficiently low, then generated photons will find the cone of acceptable escape angles too narrow to produce significant light extraction. Fig. 1.2(a) shows this narrow cone of acceptance as a red cone pointing normal to the semiconductor surface. Any photon outside of that cone in that coordinate axis will be reflected internally, thereby decreasing light extraction. 


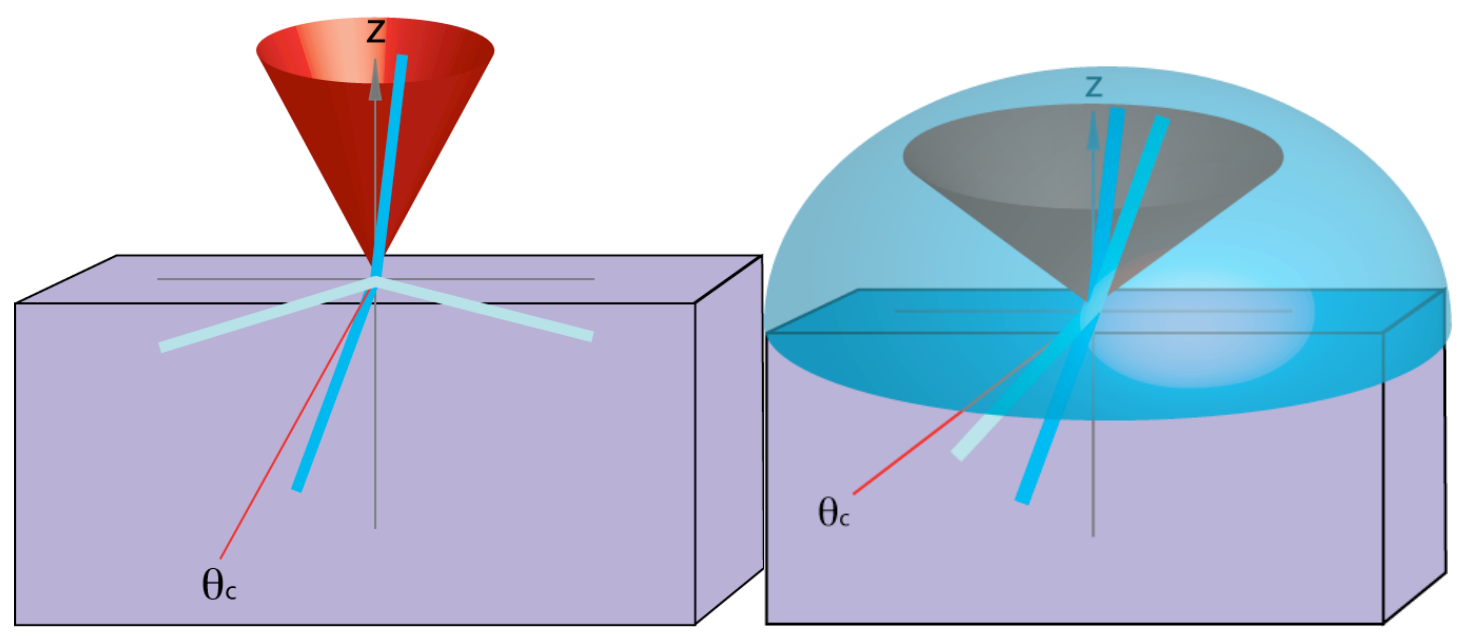

(a)

(b)

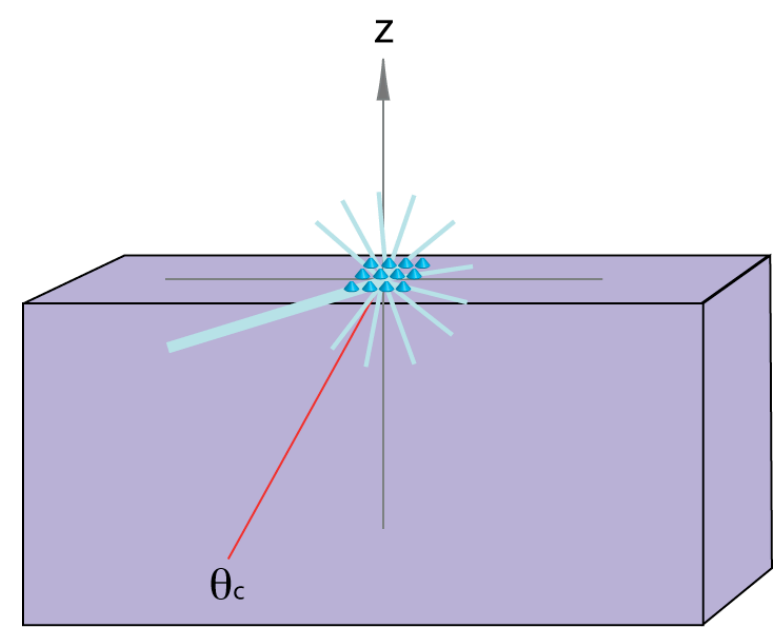

(c)

Fig. 1.2 - Total Internal Reflection Model: (a) cone of transmitted angles and total internal reflection, (b) LED with enhanced cone of transmitted angles, due to affixed resin dome, and (c) incident beam scattering via Bragg diffraction at the grating interface

A number of solutions have been proposed to solve this problem. First, a common approach affixes a dome-shaped resin over the LED to create a larger escape angle out of the device. Fig. 1.2(b) shows how the critical angle might be increased and therefore increase light extraction efficiency. The red cone of transmitted angles increases when compared to Fig. 1.2(a). This results because of the lower index difference between GaN 
and the resin. In addition, the resin's dome shape ensures that the extracted light at the semiconductor-resin interface meets the resin-air interface at a normal or a low enough angle to allow for greater light extraction [5]. To further mitigate the problems of total internal reflection, the emission surface can be patterned to form a transmission grating that offers the light more angles of escape, as shown in Fig. 1.2(c). A transmission grating allows partial transmission at angles above the critical angle. Instead of light reflecting at grating surfaces, the light instead scatters at these surfaces. A variety of methods, including wet etching with an amorphous sacrificial layer [6-9] or by laser etching to obtain a more periodic spacing [10-14], can etch a transmission grating on a semiconductor surface. In previous literature, the same patterning can also apply, with a similar effect, to an $\mathrm{Ag}$ reflector plate in either pillar or hole grating shapes to form a reflection grating [15-16]. Finally, if one could exchange the semiconductor for a polymer, such as polyphenylene vinylene (PPV), polyfluorene, or poly(N-vinylcarbazole) (PVK), the critical angle could be reduced, due to a polymer's lower index of refraction. For example, PVK possesses an index of refraction of 1.68 compared to GaN's 2.55. If PVK were used instead of GaN, the critical angle could be enlarged from $23^{\circ}$ to $37^{\circ}$, allowing an extra $14^{\circ}$ of light to escape out of the LED [42].

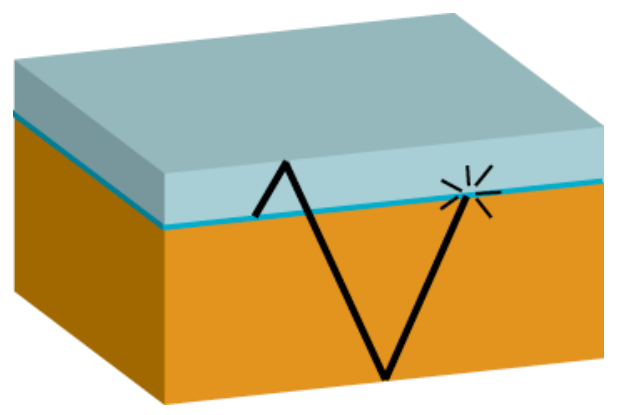

Fig. 1.3 - Absorption within the LED 
A light emissive device's second key limitation results from the absorption of generated light within the device at the multiple quantum well region or at defects within the GaN crystal structure, as shown in Fig. 1.3. The top layer is a p-GaN semiconductor, followed by a MQW active layer, and an $\mathrm{n}-\mathrm{GaN}$ semiconductor. The photon that cannot escape due to an incident angle higher than the critical angle travels within the LED and eventually absorbs into the MQW active layer. Photon absorption constitutes a critical aspect of not only light extraction efficiency, but also of device operation. Since the photon does not escape from the device, it also does not contribute to the maximization of light output in lumens per watt. In addition, the absorbed photon generates heat that is dispersed throughout the diode through a phonon interaction or lattice vibration [39]. Increased heat within an LED luminaire seriously degrades LED lifetime, alters the emitted wavelength of light, and consumes energy that could be used for light emission. The MQW region generates the light within the diode and deserves particular attention. Since the generated photon matches the bandgap energy of the light-generating, MQW layer, it can readily absorb photons and does so at a high rate. Fig. 1.3 illustrates how every pass across the MQW layer increases the chances of absorption. Photons must be extracted, both in greater quantity and speed, before absorption occurs [4, 17, 39]. A grating structure will solve this issue by creating more angles of escape. Grating structures can be patterned into a plethora of shapes, including: pyramidal, spherical, conical, cylindrical, and many other shapes, but only a few can be fabricated with great success. For example, the modified laser-lift off (M-LLO) technique can pattern holes a 4-micron period instead of etching a random structure. The technique etches the 
nanostructure onto $\mathrm{u}-\mathrm{GaN}$ using a sapphire backplane, UV light, and a high power $\mathrm{KrF}$ laser. Peking University's (PKU) experiment varied the grating depths from $75 \mathrm{~nm}$ to 120nm [18]. Imprint lithography, that can also produce similar air holes that measure $180 \mathrm{~nm}$ in diameter, with a depth of $67 \mathrm{~nm}$, and a period of $295 \mathrm{~nm}$ [19].

The light trapping issue is commonly solved by etching a structure on the extraction surface and/or on the bottom reflection surface. The single grating simulation has been studied intensively already, but there exist, to this date, few studies on multiple gratings in the same device. It is also not practical to fabricate all the double grating cases to obtain the best cases. Even this thesis is only limited to one particular GaN LED structure developed by Peking University [9]; we simulate at least 181 cases based on the microstructure gratings and present very interesting results. In addition, etching also involves positional defects, and so the grating structure is not a perfectly periodic crystal. We also develop an error-grating model to study the effects of positional defects during fabrication. To date, there is no publication on the error-grating model of the top and bottom grating structures on GaN LEDs. We calculate the effects of different grating structure combinations on light extraction efficiency using a Finite Difference Time Domain (FDTD) method.

The research presented in this thesis simulates a variety of grating types, explores the transmission of light about the critical angle, and uncovers the mechanics behind light extraction from grating structures. Gratings can vary in following ways: (1) by placing the grating on different layers within the thin-film LED, (2) by varying the density of grating cells on the grating layer, (3) by altering the shape of the grating cells, and (4) by 
increasing the symmetry of the photonic crystal grating. Single grating structures approximately double the light extraction from an LED. Double grating structures may outperform single grating structures. However, optimization must be performed with both gratings together and to optimize the grating structures. We can discover absolute maxima by using a genetic mutation algorithm in the future work. For the purposes of this thesis, we only find local maxima that are better than either single grating alone. In our simulations on the best simulated top and bottom grating case, we found that a single variable optimization procedure could increase the light extraction efficiency by $53 \%$ over the original unoptimized case. In addition, we simulated error grating models that represented positional-perturbed grating cells as a result of fabrication defects. Our findings show that low intensity perturbation is not only beneficial for light extraction, but in many cases even desirable. Finally, we experiment on 2-fold symmetric photonic crystals and 3-fold symmetric GaN LED models and find that diffraction gratings not only aid in light extraction for angles past the critical angle, but also are the mechanism for light escape from an LED surface in micron-scale gratings.

We organize the thesis chapters as follows: Chapter 2 details the FDTD method that is the primary tool for this simulation research, Chapter 3 presents the results of the single reflection grating studies, Chapter 4 presents the results of the top transmission grating studies, Chapter 5 demonstrates for the first time simulation studies in top and bottom grating simulations and subsequent partial optimization, and Chapter 6 explores a novel method for studying fabrication defects in ordered photonic crystals of top and bottom grating structures. Chapter 7 explores the transmitted diffraction simulation and 
experiment of a patterned PDMS hemicylinder experiment. Chapter 8 employs the use of a 3-D FDTD simulation to study 3-fold symmetric photonic crystal structures. Finally, Chapter 9 summarizes the major conclusions of each chapter and also presents and an extensive list of future research for this project, which include nano-structure gratings, photonic quasicrystals gratings, and nano-bump defects.

\subsection{International Collaboration with Peking University}

Peking University (PKU), in Beijing, China, conducted the experimental part of this research. The collaboration allowed research of both simulation and experiment in a cost effective manner. Peking University boasts of over 100 years of academic history as a major research university, while maintaining their status as the top university in China. Cal Poly entered into this collaborative agreement with PKU's State Key Laboratory for Mesoscopic Physics and Department of Physics in July 2007 with the intention of having

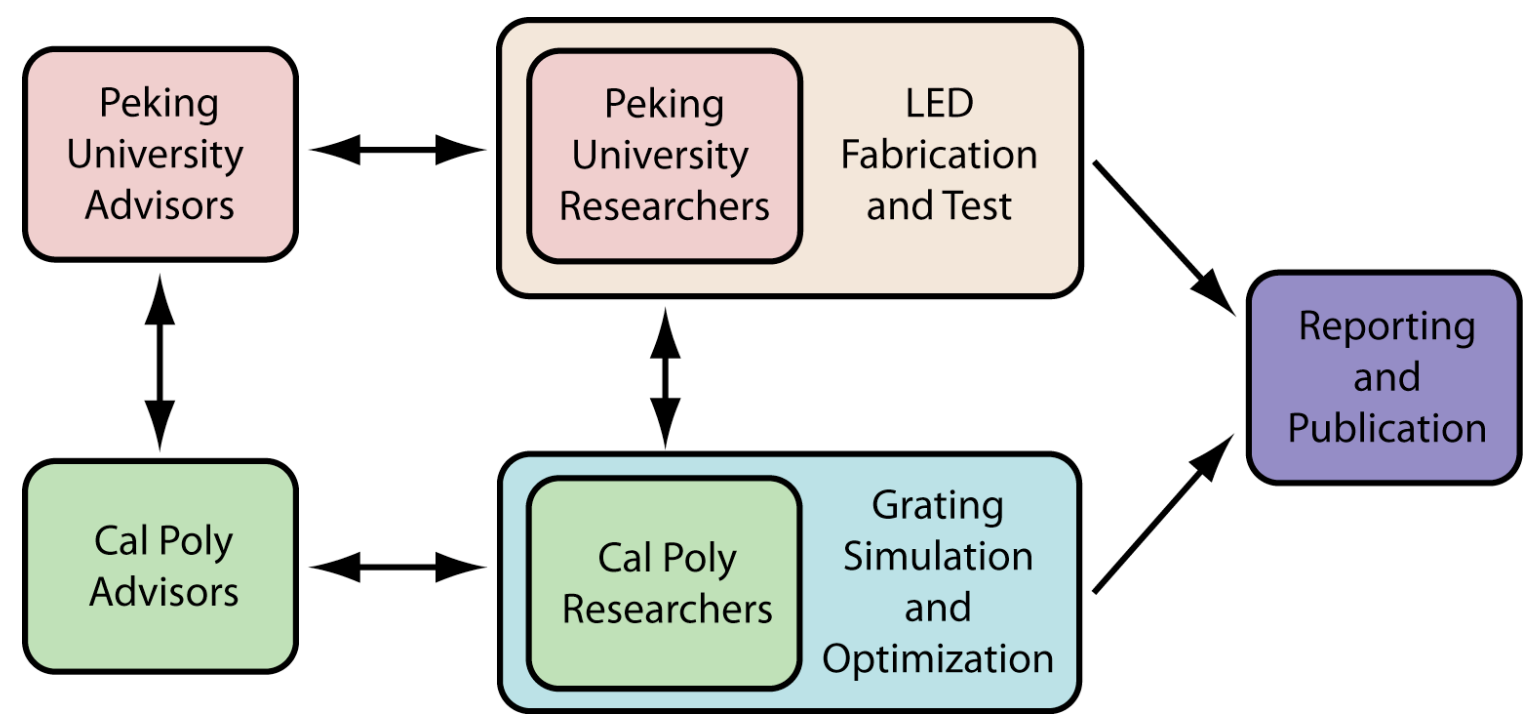

Fig. 1.4 - International Collaboration Agreement with Peking University and Cal Poly 
Cal Poly students perform grating optimization simulations, while PKU would prepare samples and perform light output measurements. We would report on our research efforts together in conference papers, journals and other informal publications and presentations. Advisors from both universities would direct student research, while communicating with each other for the direction of the project, as shown in Fig. 1.4, a helpful visualization of the international collaboration agreement. Students would also collaborate and sometimes travel to each respective university to discuss research goals and clarify results or conclusions in the English or Chinese language. If the research had significant findings, we would publish papers together and send a representative to present the results of our collaborative research. Cal Poly's strength in electrical engineering and simulation, coupled with PKU's expertise in device physics and fabrication, built a strong foundation for research in GaN LEDs. 


\section{Chapter 2 - The Finite Difference Time Domain Method}

Computational electromagnetic (CEM) simulation has offered researchers powerful tools to observe optical phenomenon without having to actually construct a physical device. These algorithms all base their calculations on some form of Maxwell's equations. For example, the transfer-matrix method (TMM), a surface-based method, calculates the propagation of electromagnetic fields in multi-layered medium, accounting for both transmission and reflection at the interfaces. This method utilizes the properties of Maxwell's equations to define the continuity of the electric field across each boundary. However, TMM finds no use for situations where the interfaces do not represent a flat medium, since it only takes into account the reflections at a flat interface. A rigorous GaN LED model must be able to 1) model an LED as a layered medium and 2) account for interactions with elements of arbitrary shape and size (ie. gratings). The Finite Difference Time Domain (FDTD) method accomplishes all of these purposes and derives from the differential form of Maxwell's equations. The FDTD method does not approximate field solutions, and therefore, provides a mature, rigorous solution to electromagnetic field propagation [20]. The method has already found versatile applications in many fields, including ring resonators, scattering from biological cells, ground-penetrating radar, antennas, WLANs, and wireless systems [21-24].

\subsection{Derivation of FDTD Theory}

We analyze a GaN LED device by the FDTD method because of the significant advantages listed in the previous paragraph [25]. This section of the thesis provides the 
theoretical background to understand how fields propagate in the simulation domain of any device model presented herein. The presentation begins with the differential forms of Maxwell's equations in Eq. 2.1a and Eq. 2.1b:

$$
\begin{aligned}
& \frac{\partial \vec{H}}{\partial t}=-\frac{1}{\mu}(\nabla \times \vec{E}) \\
& \frac{\partial \vec{E}}{\partial t}=\frac{1}{\varepsilon}(\nabla \times \vec{H})-\frac{\sigma}{\varepsilon} \vec{E}
\end{aligned}
$$

We break up these equations into the time-differentiated, spatial components of the curl operation of each respective axis in the Cartesian coordinate system, which results in the set of equations below in Eq. 2.2:

$$
\begin{aligned}
& \frac{\partial H_{x}}{\partial t}=-\frac{1}{\mu}\left(\frac{\partial E_{z}}{\partial y}-\frac{\partial E_{y}}{\partial z}\right) \\
& \frac{\partial H_{y}}{\partial t}=-\frac{1}{\mu}\left(\frac{\partial E_{x}}{\partial z}-\frac{\partial E_{z}}{\partial x}\right) \\
& \frac{\partial H_{z}}{\partial t}=-\frac{1}{\mu}\left(\frac{\partial E_{x}}{\partial y}-\frac{\partial E_{y}}{\partial x}\right) \\
& \frac{\partial E_{x}}{\partial t}=\frac{1}{\varepsilon}\left(\frac{\partial H_{y}}{\partial z}-\frac{\partial H_{z}}{\partial y}-\sigma E_{x}\right) \\
& \frac{\partial E_{y}}{\partial t}=\frac{1}{\varepsilon}\left(\frac{\partial H_{x}}{\partial z}-\frac{\partial H_{z}}{\partial x}-\sigma E_{y}\right) \\
& \frac{\partial E_{z}}{\partial t}=\frac{1}{\varepsilon}\left(\frac{\partial H_{x}}{\partial y}-\frac{\partial H_{y}}{\partial x}-\sigma E_{z}\right)
\end{aligned}
$$

We can easily solve for each field component in all three spatial directions for all field parameters. For example, the first line in Eq. 2.2 presents the time differential form of the $\mathrm{H}$-field component in the $\mathrm{x}$-direction and can be directly solved with only a few E-field 
components in the $\mathrm{z}$ - and $\mathrm{x}$-directions and the previous field value. We attribute the original application of this technique to Dr. Kane Yee in 1966, in which he used an interleaved mesh of $\mathrm{E}$ and $\mathrm{H}$ field components, the so-called Yee's mesh shown in Fig. 2.1 below. The interleaving component fields allow us to use the adjacent fields surrounding the desired field to perform the calculation. Each H component offsets by $n+1 / 2$ while the E component offsets by $n+1$. The field components essentially "leapfrog" each other, alternating between $\mathrm{E}$ and $\mathrm{H}$ field components, as shown in Eq. 2.3a to Eq. 2.3f. Each cell also has a differential $\Delta \mathrm{x}, \Delta \mathrm{y}$, and $\Delta \mathrm{z}$ component, all of which define the size of the mesh. The mesh sizes have profound effects on the simulation domain, simulation time, and memory requirements, which we will see later in section 2.2.

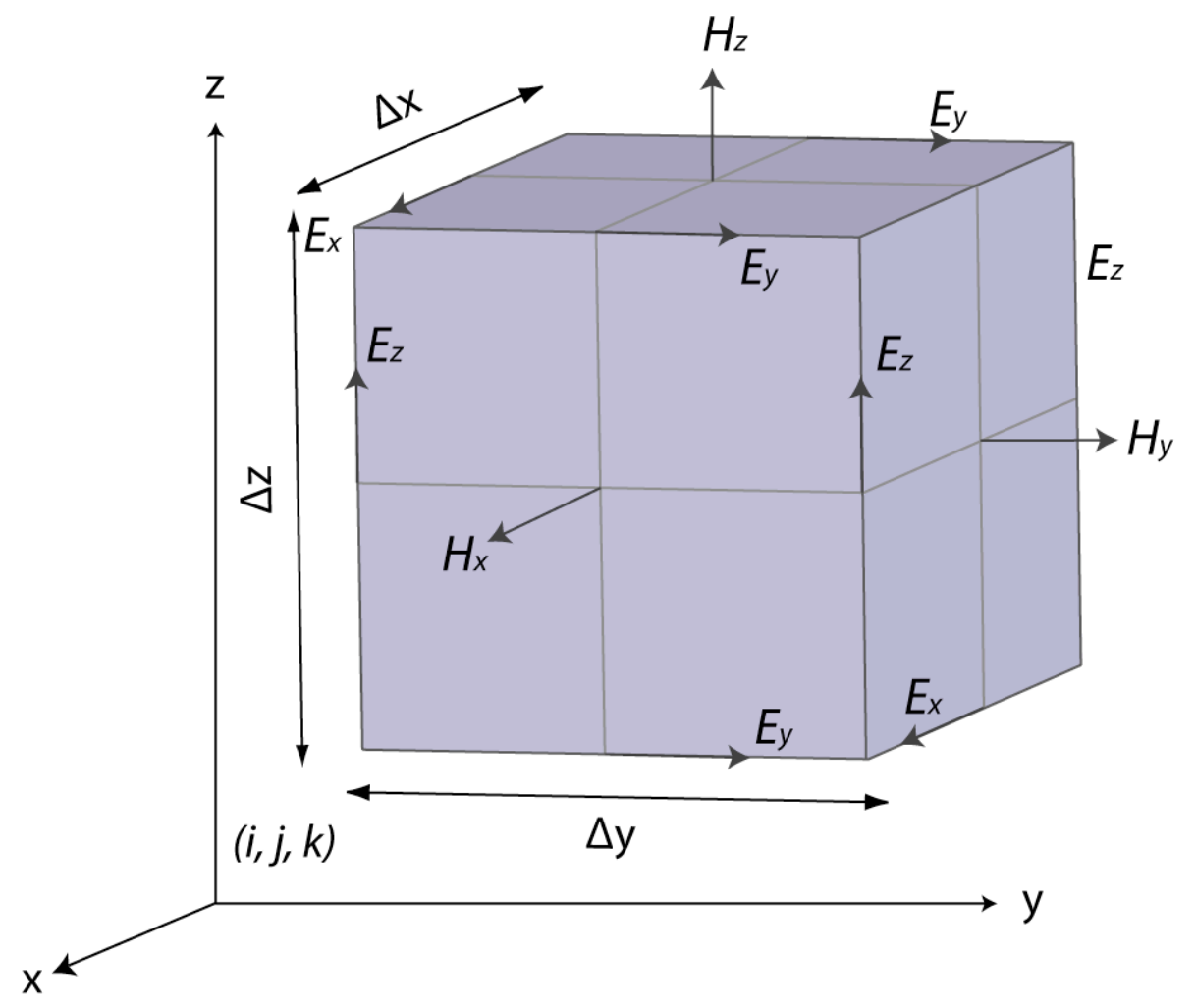

Fig. 2.1 - Yee's Mesh 
Maxwell's differential form, with a Yee's mesh implementation, decomposes into the following "leapfrogging” equations:

$$
\begin{aligned}
& H_{x}^{n+1 / 2}(i, j+1 / 2, k+1 / 2)=H_{x}^{n-1 / 2}(i, j+1 / 2, k+1 / 2)-\frac{\Delta t}{\mu(i, j+1 / 2, k+1 / 2)}\left[\begin{array}{l}
\frac{E_{y}^{n}(i, j+1 / 2, k+1)-E_{y}^{n}(i, j+1 / 2, k)}{\Delta z} \\
-\frac{E_{z}^{n}(i, j+1, k+1 / 2)-E_{z}^{n}(i, j, k+1 / 2)}{\Delta y}
\end{array}\right], \\
& H_{y}^{n+1 / 2}(i+1 / 2, j, k+1 / 2)=H_{y}^{n-1 / 2}(i+1 / 2, j, k+1 / 2)-\frac{\Delta t}{\mu(i+1 / 2, j, k+1 / 2)}\left[\begin{array}{c}
\frac{E_{x}^{n}(i+1 / 2, j, k+1)-E_{x}^{n}(i+1 / 2, j, k)}{\Delta z} \\
-\frac{E_{z}^{n}(i+1, j, k+1 / 2)-E_{z}^{n}(i, j, k+1 / 2)}{\Delta x}
\end{array}\right), \\
& H_{z}^{n+1 / 2}(i+1 / 2, j+1 / 2, k)=H_{z}^{n-1 / 2}(i+1 / 2, j+1 / 2, k)-\frac{\Delta t}{\mu(i+1 / 2, j+1 / 2, k)}\left[\begin{array}{l}
\frac{E_{y}^{n}(i+1, j+1 / 2, k)-E_{y}^{n}(i, j+1 / 2, k)}{\Delta x} \\
-\frac{E_{x}^{n}(i+1 / 2, j+1, k)-E_{x}^{n}(i+1 / 2, j, k)}{\Delta y}
\end{array}\right), \\
& E_{x}^{n+1}(i, j+1 / 2, k+1 / 2)=\left[1-\frac{\sigma(i, j+1 / 2, k+1 / 2)}{\varepsilon(i, j+1 / 2, k+1 / 2)}\right] E_{x}^{n}(i, j+1 / 2, k+1 / 2)+\frac{\Delta t}{\varepsilon(i, j+1 / 2, k+1 / 2)}\left[\begin{array}{l}
\frac{H_{y}^{n+1 / 2}(i, j+1 / 2, k+1)-H_{y}^{n+1 / 2}(i, j+1 / 2, k)}{\Delta z} \\
-\frac{H_{z}^{n+1 / 2}(i, j+1, k+1 / 2)-H_{z}^{n+1 / 2}(i, j, k+1 / 2)}{\Delta y}
\end{array}\right],
\end{aligned}
$$

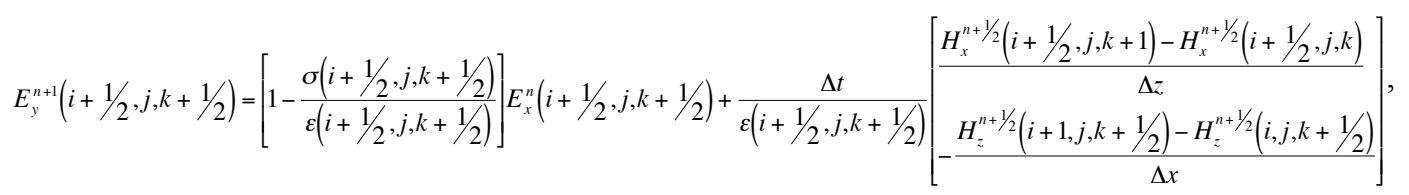

$$
\begin{aligned}
& E_{z}^{n+1}(i+1 / 2, j+1 / 2, k)=\left[1-\frac{\sigma(i+1 / 2, j+1 / 2, k)}{\varepsilon(i+1 / 2, j+1 / 2, k)}\right] E_{x}^{n}(i+1 / 2, j+1 / 2, k)+\frac{\Delta t}{\varepsilon(i+1 / 2, j+1 / 2, k)}\left[\frac{H_{x}^{n+1 / 2}(i+1 / 2, j+1, k)-H_{x}^{n+1 / 2}(i+1 / 2, j, k)}{\Delta y}\right],
\end{aligned}
$$

where $\mathrm{i}, \mathrm{j}$, and $\mathrm{k}$ are indexes indicating position within the simulation domain, $\sigma$ is the conductance, $\mu$ is the permeability, and $\varepsilon$ is the permittivity. The indexes determine what material to use according to the coordinates $(i, j, k)$ plus any additional offset (ie. $+/-1 / 2)$ defined by the Yee's mesh. Also, other grid geometries can be formulated, but the software used at Cal Poly limits us to only cubic meshes [25]. 


\subsection{Accuracy and Stability of the FDTD Method}

We can and should define the coarsest grid size possible to achieve accurate results, since simplification to the model can result in gigabytes of saved memory and hours of saved simulation time. The grid size should be at least as small as the smallest element in the simulation. For example, if a quantum well region is $67 \mathrm{~nm}$ tall, then the grid size in the direction of the quantum well layer thickness should be at most $67 \mathrm{~nm}$, or $\Delta \mathrm{z}<67 \mathrm{~nm}$. Another rule of thumb states that the grid sizes should be no larger than one tenth of the wavelength used in the simulation. In addition to these accuracy requirements, we must satisfy the Courant stability condition, shown in Eq. 2.4,

$$
\Delta t=\frac{1}{c \sqrt{1 /(\Delta x)^{2}+1 /(\Delta y)^{2}+1 /(\Delta z)^{2}}},
$$

which states that a decrease in the Yee's mesh dimensions will result in a smaller time step requirement. For example, if each differential distance were $1 \mathrm{~m}$, then the time step would be $1.92 \mathrm{~ns} / \mathrm{step}$. But suppose we halved only one differential distance to 0.5 ; then the time step would change from $1.92 \mathrm{~ns} / \mathrm{step}$ to $1.36 \mathrm{~ns} / \mathrm{step}$, resulting in a simulation time increase of $29 \%$, a sizeable difference in time. By this calculation, if a simulation took 10 hours to complete with the original grid size, then just cutting one of the differential distances in half results in the simulation now taking 12.90 hours to complete. Also, Eq. 2.4 reveals that the stability conditions vary sensitively with each individual differential dimension, therefore the grid can take a rectangular shape, as well as a square shape. So, the correct balance that maximizes the differential distances but maintains accuracy and stability of the simulation provides the most time and memory efficient 
solution for an FDTD simulation [25].

\subsection{Computational Requirements of FDTD}

The small changes in grating parameters require the use of a CEM method, such as FDTD, that can accurately simulate the effects of refraction in device materials, reflection due to linear dispersion or total internal reflection, transmission of escaping light from the LED, and scattering at the grating. The FDTD method combined with a Yee's mesh can simulate structures of arbitrary length and size. However, the constraints of the model limit the size, due to the simulation time and amount of memory required to simulate larger devices. FDTD implementers have developed many techniques to ease the requirements of three-dimensional models including the use of Graphics Processing Units (GPU), parallel processing, and mode simplifications [26-28]. To give a better idea of how computational requirements scale, three-dimensional model parameters scale in simulation time like $\mathrm{N}^{4}$ and in memory like $\mathrm{N}^{3}$. For example, a 2-fold increase in the simulation domain in all three dimensions with no change in the differential grid sizes would result in a 16X increase in simulation time and an $8 \mathrm{X}$ increase in RAM requirements. Clearly, the demands for FDTD computing resources erect a high technological barrier. For this research, we use the following hardware: 8 -core $2.8 \mathrm{GHz}$ Intel Xeon microprocessor and 14 GB 800 MHz DDR2 FB-DIMM RAM. These resources provide more than enough to simulate a large device of surface dimensions $100 \times 100 \mu \mathrm{m}^{2}$ in $2-\mathrm{D}$ simulations or a 3-D simulation with a model using symmetry-based simplifications. We employ the two-dimensional FDTD to simulate this model to avoid 
unnecessary complications for most simulations requiring only 2-fold symmetric photonic crystal structures. If the model requires higher symmetries, then we must use a full 3-D simulation [29]. In particular, when simulating resource-intensive, 3-D FDTD models, this research uses the method of parallel processing to reduce the research cycle time.

\subsection{RSoft Photonics Suite ${ }^{\mathrm{TM}}$ and the FullWAVE ${ }^{\mathrm{TM}}$ Module}

RSoft Design Group Inc. develops the program used to calculate light output from an LED, and their FDTD product, FullWAVETM, forms a partial component of their broader photonic component design suite, which has the capabilities of simulating passive devices, active devices, and other specific applications. According to the company's product page, FullWAVETM can allow for "rapid virtual prototyping reducing the need for costly and lengthy physical prototyping increasing productivity and decreasing time-tomarket, automated parameter scanning via the MOST ${ }^{\mathrm{TM}}$ optimizer, and discovery of new products by creating 'what if' product scenarios" [30]. These features speak for themselves, particularly when we must consider many small changes to a grating structure over the difference of a few nanometers at a time. The FullWAVETM module affords us great flexibility in researching many combinations of grating parameters for automated optimization and test. 


\subsubsection{Construction of Thin-film LEDs with the RSoft CAD Environment}

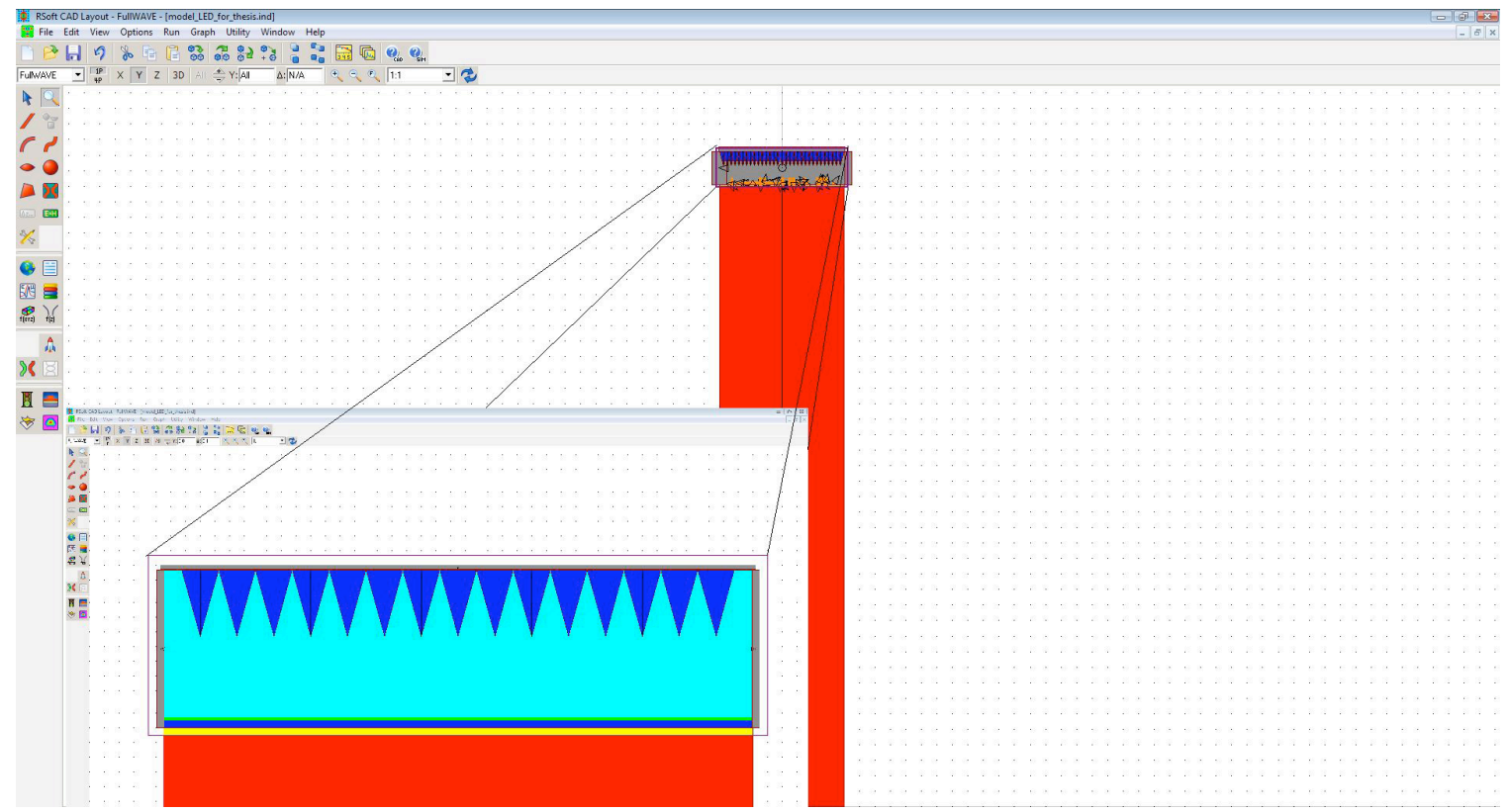

Fig. 2.2 - RSoft CAD Environment Window

FullWAVETM performs FDTD calculations, but we must draw the actual design of the thin-film LED is drawn up in the RSoft Computer Aided Design (CAD) Environment ${ }^{\mathrm{TM}}$ (v. 8.1). Shapes of many kinds can be placed on a simulation domain, including rectangular cubes, cones, cylinders, lenses, and other shapes important to photonic device design. Associated material properties link with each shape. Fig. 2.2 shows the general layout screen of the CAD environment with a superimposed, zoomed in figure of a flipchip GaN LED model with a conical hole transmission grating structure. One can use the left toolbar to choose an element to draw in the simulation domain. 
Table 2.1 - Segment Properties with Descriptions

\begin{tabular}{ll} 
Segment Property & Description \\
\hline 3D Structure Type & $\begin{array}{l}\text { The shape of the segment } \\
\text { The merge priority in case of overlap (ie. higher numbers have higher } \\
\text { priority) }\end{array}$ \\
Merge Priority & The color displayed for the segment in CAD \\
Dispay Color & The orientation of the segment, usually z-axis \\
Seg Orientation & Selects the type of material to use \\
Material Properties & Index of refraction (may subtract out background index, depending on \\
Index Difference & setup) \\
Index (imag part) & Imaginary part of the index of refraction \\
Component width & Segment width in the x direction \\
Component height & Segment height in the y direction \\
Reference type & What reference to use for the length \\
Offset value & The offset from the reference to use to determine the length \\
Relative to Component & The component it's relative to, usually the same component \\
\hline
\end{tabular}

A placed structure can then be modified to fit a particular shape, index profile, merge priority, display color, segment orientation, width taper, height taper, material property, exact position in the simulation domain, and shape dimension through the segment properties window, shown in Fig. 2.3. Also, Table 2.1 gives a brief summary of each defining property along with its most common usage. One must define the correct 3D Structure Type to draw a particular type of segment (ie. cone, cylinder, rectangular cube, etc.), Table 2.2 shows the types of shapes that can be drawn along with the "3D Structure Type" to use. As an example, a "Fiber" structure type (ie. fiber optic) can create a cylinder shape useful for creating cylindrical grating cells. We define the height by setting the z-reference to an "Offset" type and also define an offset length relative to a certain component, most often itself. We set the width using the "Component Width" and "Component Height" parameters in the "Starting Vertex" and "Ending Vertex" titled borders. Entering a width and height into these fields will expand or contract the segment along the $\mathrm{x}$ - and $\mathrm{y}$-axis. To create a cone shape instead of a cylindrical shape, we merely 
change either end of the vertex to a width and height of 0 , depending on the desired orientation (ie. top facing or bottom facing). CAD files use the filename extension of *.IND in ASCII format. An ASCII file format lends itself to automated procedures, such as generating $\mathrm{CAD}$ elements, which we will take advantage of in section 2.5 of this thesis.

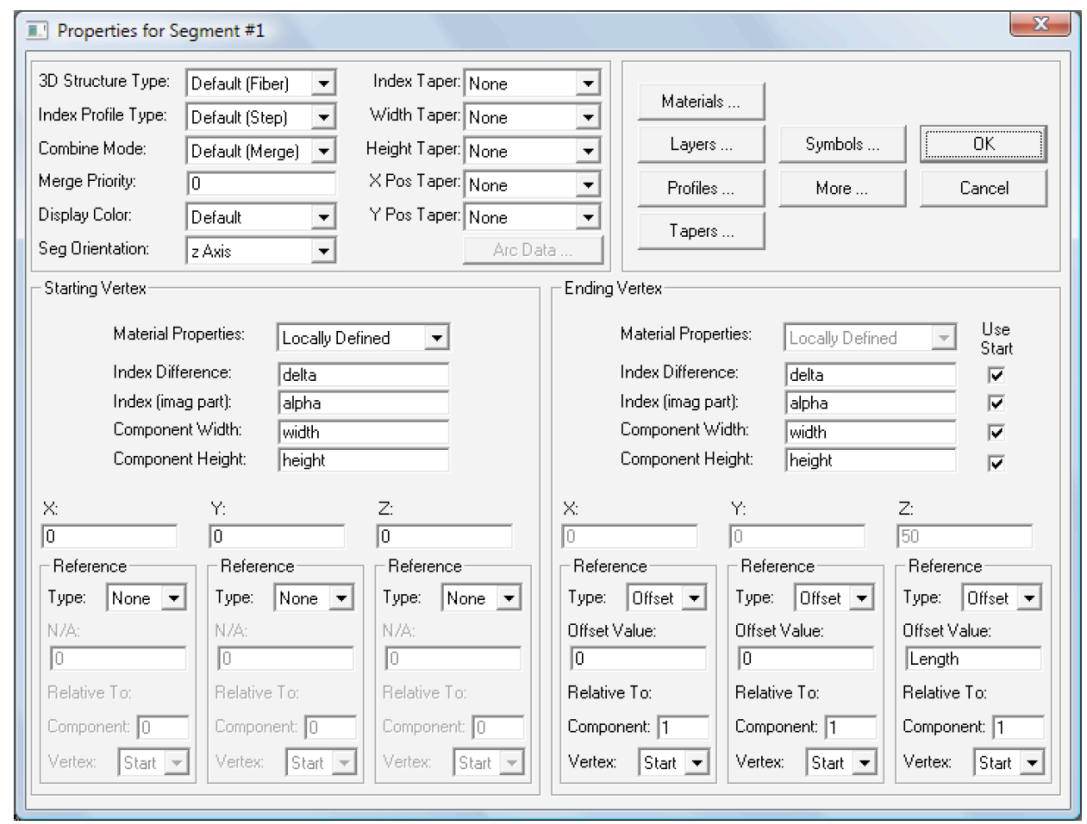

Fig. 2.3 - Segment Properties Window

Table 2.2 - 3-D Structure Types

\begin{tabular}{ll} 
3D Structure Type & Application \\
\hline Fiber & Grating cells (ie. conical or cylindrical), fiber \\
Channel & Thin-film layers, waveguides \\
Diffused & To diffuse an index profile \\
Rib/Ridge & One dimensional wave guides \\
Multilayer & Multilayered rib structure \\
\hline
\end{tabular}

\subsubsection{Grid Sizes for Accuracy and Stability in RSoft FullWAVE ${ }^{\text {TM }}$}

The differential distances, $\Delta \mathrm{x}, \Delta \mathrm{y}$, and $\Delta \mathrm{z}$, represent the grid sizes shown in the Yee's 
mesh in Fig. 2.1. A smaller grid size produces a more accurate simulation, especially at material interfaces, such as a semiconductor-air interface used in this simulation. But, smaller grid sizes produce a higher density of calculation points in the simulation domain, which increases the amount of memory required to store such a vast matrix of field data. Also, the Courant stability condition tells us that smaller grid sizes result in significantly longer simulation times. Finding appropriate grid sizes constitute a critical aspect of a model's feasibility. Fig. 2.4 shows the FullWAVE'TM Simulation Parameters dialog that has fields for setting grid sizes and time steps. Titled borders for the $\mathrm{X}, \mathrm{Y}$, and $\mathrm{Z}$ axis each contain a field for a "Grid Size" that should be no larger than smallest dimension on that axis or one tenth of the wavelength of light used in the simulation, picking the smaller of the two. A medium sized grid should encompass the simulation domain with an appropriately dense set of grid points to ensure simulation accuracy. However, an FDTD simulation can, surprisingly, accurately simulate a device model with a relatively coarse grid, as well. Stability and accuracy tests can be performed on smaller models to determine the appropriate gridding. FullWAVETM addresses stability issues by having a recommended value calculated from the Courant stability condition in the uneditable field, the "Stability Limit", which should not be exceeded. In fact, FullWAVETM will not let the simulation run if the FDTD time step exceeds the stability limit. The simulation's time step, $\Delta \mathrm{t}$, can be set in the "Time Step" field. If no metallic materials inhabit the simulation domain, then the time step can be set close to the stability limit. However, if metals exist in the simulation domain, such as Ag used in this simulation, then we do not recommended exceeding more than $90 \%$ of the stability limit value, and in some special 
cases even less than $90 \%$. Again, an appropriate time step that will maximize accuracy and minimize simulation time can be found through testing a smaller model.

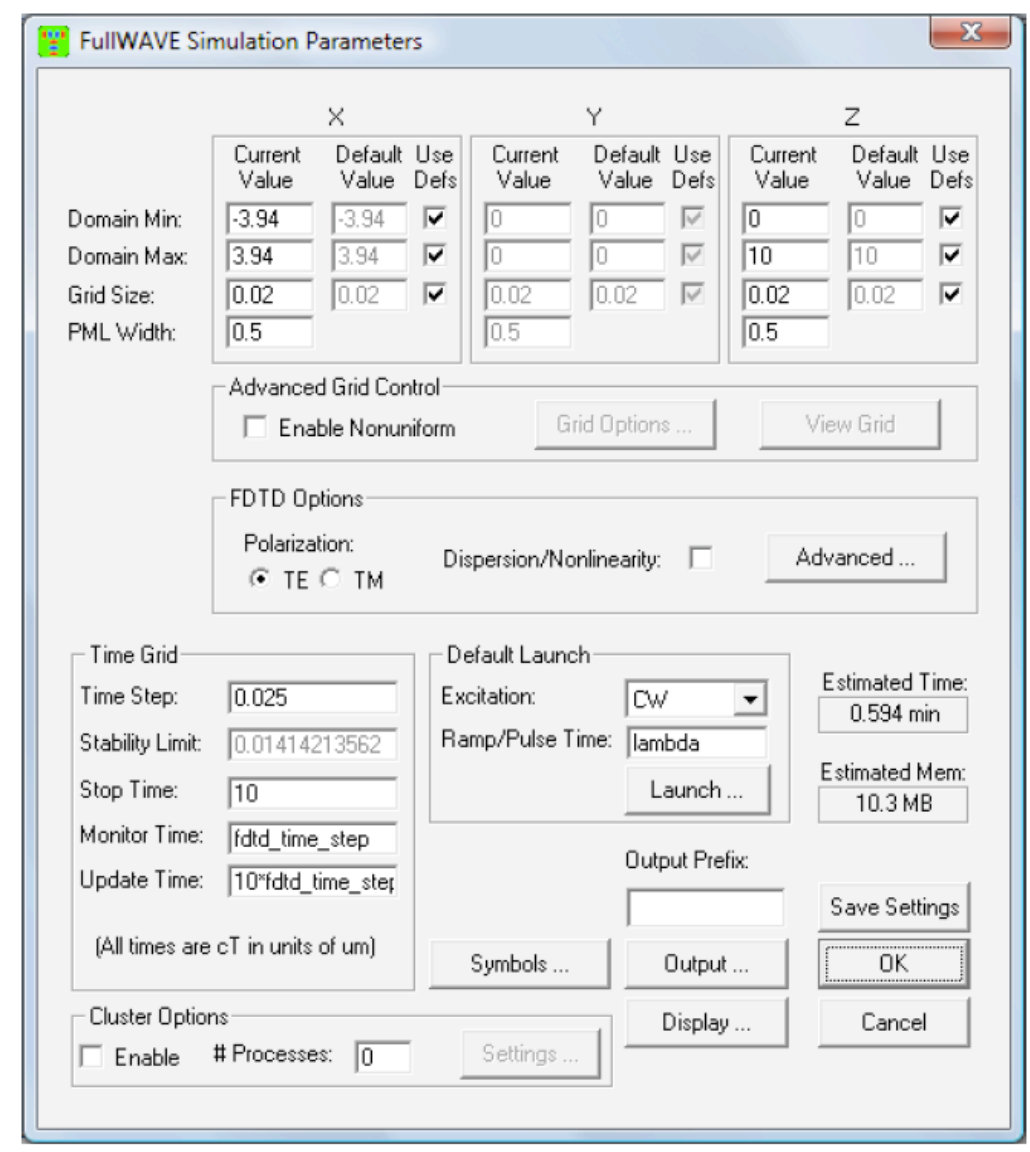

Fig. 2.4 - Simulation Parameters Window

\subsubsection{Constant Wave Sources in RSoft FullWAVETM}

Many types of excitation types can excite an FDTD simulation domain, such as impulse, pulsed, constant wave (CW), and current sources. However, most researchers simulating photonic devices use the most common source, the CW source. This type of source sends out an incident beam from the launch field rather than an introduced current term. We use this source because the multiple quantum well heterojunction layers emit 
incoherent light, that when taken on the aggregate, can be approximated by a constant plane wave, as shown in Fig. 2.5, similar to the CW source beam. Mathematically, the source can be modeled, as shown in Eq. 2.5, as a ramp up envelope, ramp down envelope, and sinusoid with a chirp factor.

$$
g(t)=\xi_{u p}(t) \xi_{\text {down }}(t) \sin \left[\frac{2 \pi}{\lambda}(t)+A t^{2}\right]
$$

where $\xi$ represents a ramping function to softly lead in and lead out a constant wave, A represents a chirp coefficient (not used in this simulation), and $\lambda$ represents the wavelength of the excitation wave. Ramping time, both up and down, was fixed to the wavelength of light in this simulation.

Multiple Quantum Wells

(Heterojunctions)

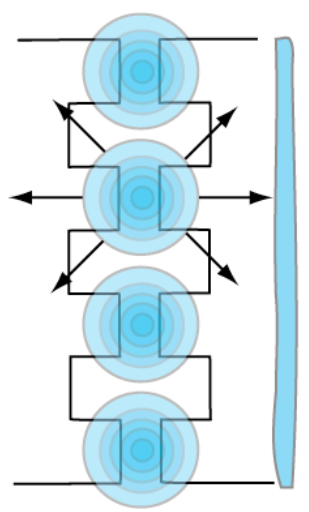

Approximated Plane Wave
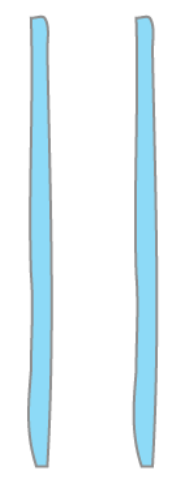

(a)

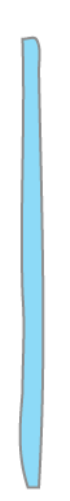$$
\text { . }
$$

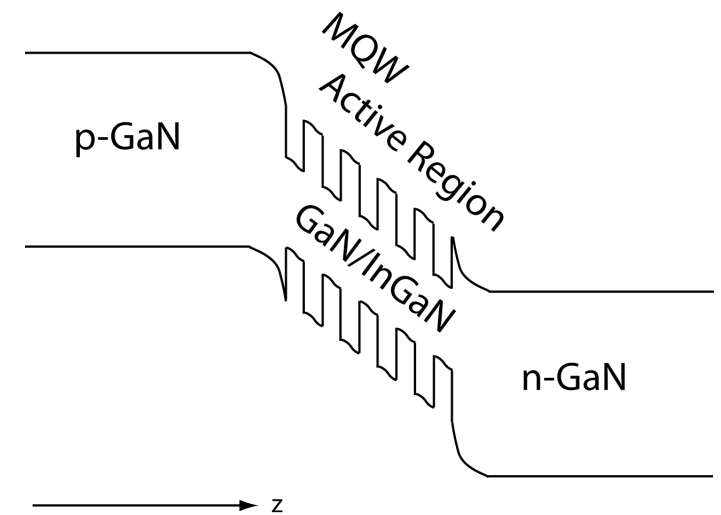

(b)

Fig. 2.5-Approximated Plane Wave: (a) emitted from the multiple quantum well region (b) band gap diagram for the GaN/InGaN heterojunction [41]

GaN, being a binary III/V direct bandgap semiconductor, emits light. GaN has a bandgap energy of $3.44 \mathrm{eV}$ and emits a wavelength in the pure blue spectrum of approximately $460 \mathrm{~nm}$ [34]. This wavelength demands particular attention, since it affects 
light extraction efficiency and grating parameter matching with internal modes.

Therefore, this parameter must be set in the "Free Space Wavelength" field in the Global Default Settings, shown in Fig. 2.6. The Free Space Wavelength in the figure shows a 1 $\mu \mathrm{m}$ wavelength simulation, but for $\mathrm{GaN}$, we must set up a $0.46 \mu \mathrm{m}$ wavelength.

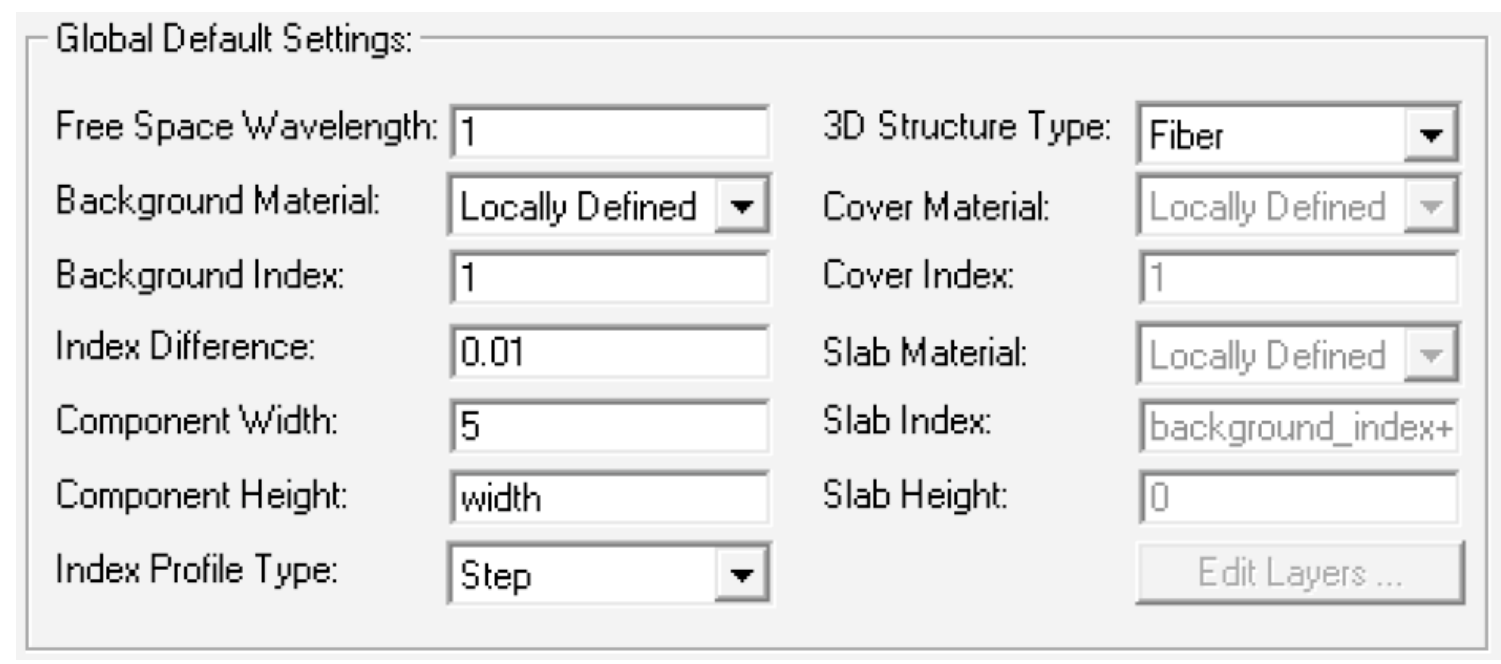

Fig. 2.6 - Global Default Settings Window

Sources within the CAD environment embed in the same way as thin-film segments. Right clicking on the source in question will bring up the "Launch Parameters" window, shown in Fig. 2.7, that allows a user to select the type of intensity distribution across the source, the orientation angle phi and theta, as well as the position in $\mathrm{X}, \mathrm{Y}$, and $\mathrm{Z}$ coordinates. Of course, the excitation will be set to a $\mathrm{CW}$ with a ramp time equal to the free space wavelength. Note that the default wavelength for the source initializes to the free space wavelength.

\subsubsection{Simulation Domain and the Perfectly Matched Layer}

Referring back to Fig. 2.4, the simulation domain defines the extent to which we want 
waves to propagate, or in the case of this thesis, the dimensions of the LED device, plus a small amount of air space to let radiating light escape from the side of the LED. The simulation domain should be no larger than necessary, as this affects the amount of memory required. More grid points must be calculated as a result of a larger simulation area. A Perfectly Matched Layer (PML), a highly absorbent that allows incident waves to exit the simulation domain with minimal reflection, surrounds the simulation region.

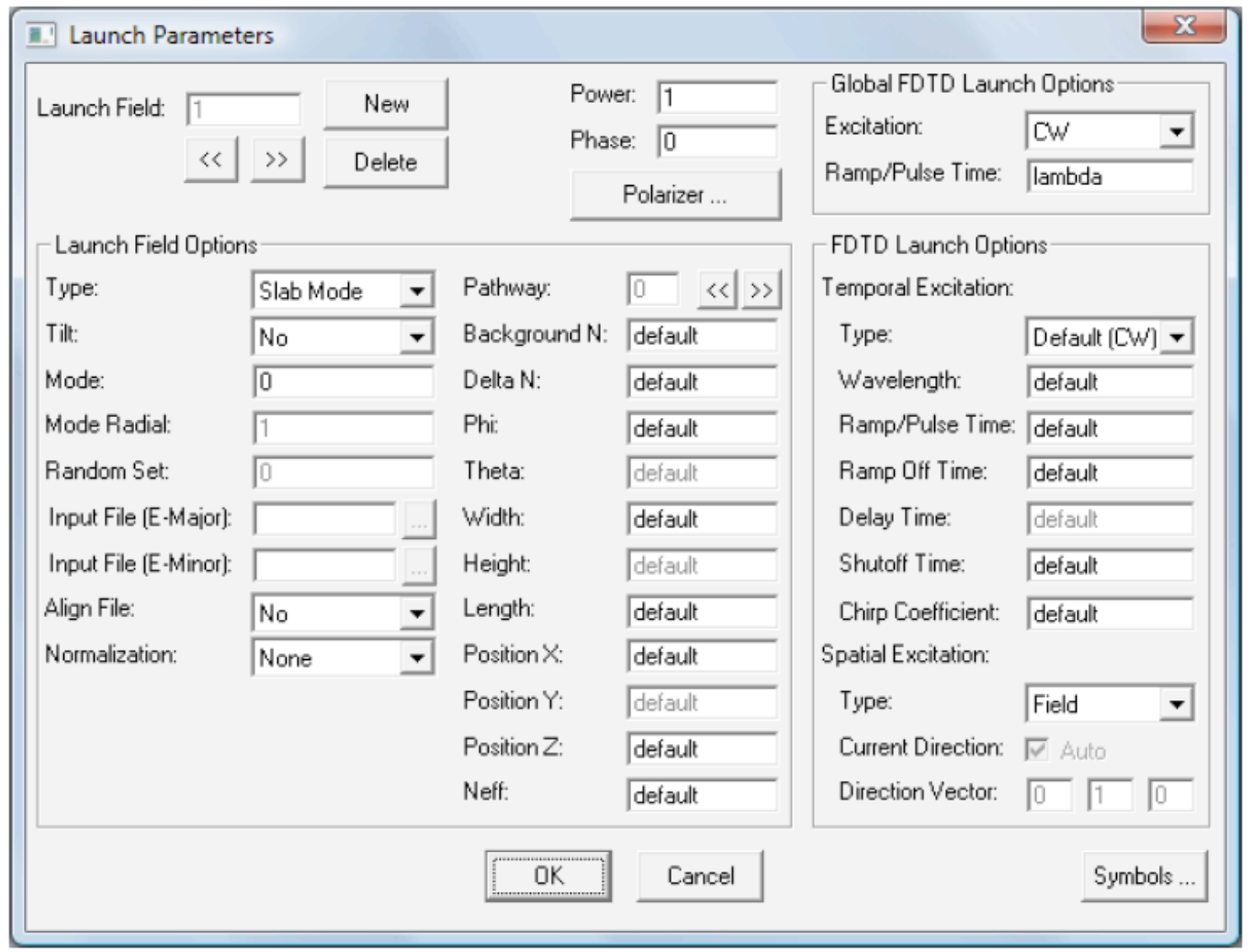

Fig. 2.7 - Launch Parameters Window

Since this extra space still forms a highly absorbent "material", the grid points added to the simulation also affect the memory requirements and simulation time, just as an 
extension to the simulation domain would also.

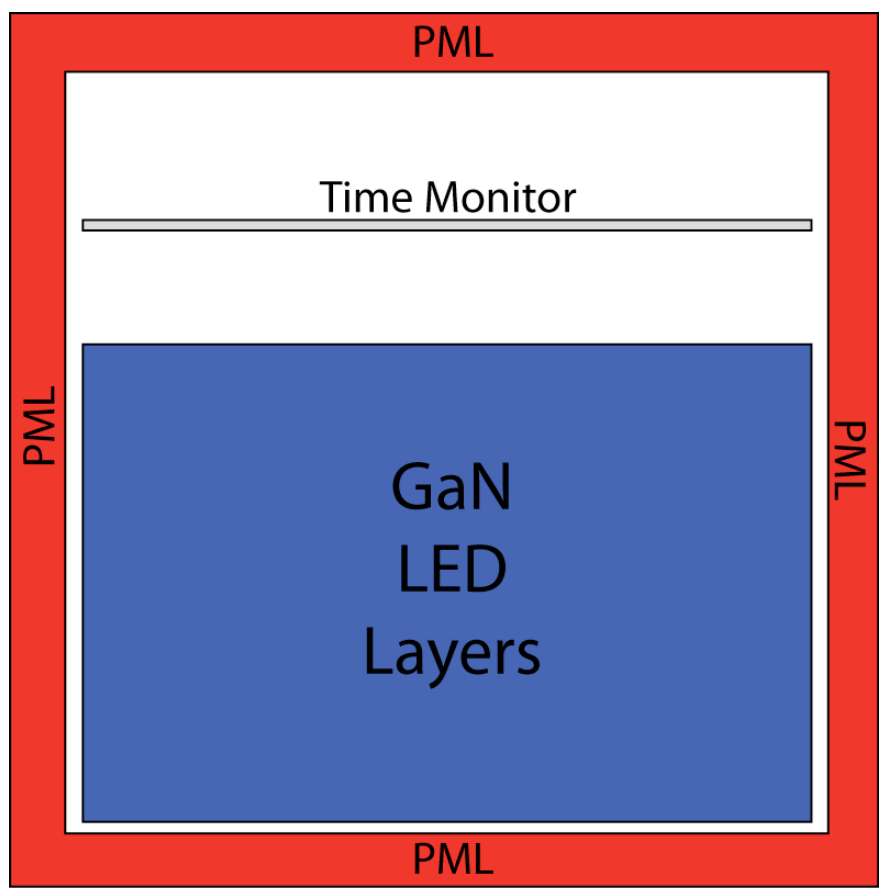

Fig. 2.8 - The PML in Reference to the Simulation Domain

To better illustrate the concept of a PML, Fig. 2.8 shows an LED encompassed in a simulation domain, surrounded by a moderate sized PML. The outermost red areas, labeled "PML", represent highly absorbent PML material, while the white area, in the simulation region enclosed by the PML, represents the simulation domain. Fig. 2.8 shows a block diagram of the GaN LED layers, as well as a time monitor to measure output power outside the LED. The PML thickness defines the thickness of the PML layer, as shown in Fig. 2.4 in the X, Y, and Z titled borders. Thicknesses measure in microns, not the SI unit of meters, due to the small size of photonic devices most often simulated in RSoft products. The thickness of the layer should be 10 grid points or more for each respective axis, as a general guideline, to allow an incident wave to have enough room to 
properly attenuate in the PML region. For example, a $\Delta \mathrm{x}$ of 1 micron means a PML in the $\mathrm{x}$-axis should be 10 microns thick.

\subsubsection{Time Monitors in RSoft FullWAVE'M}

Time monitors capture all field data in FullWAVETM. The monitor can gather many different kinds of information including spatial output, such as spatial intensity maps, farfield patterns calculated from spatial data, and Poynting vectors, as well as temporal outputs, any field component, power, field density, and absorption. One can embed a time monitor in a simulation domain by selecting the time monitor from the button on the left toolbar and placing the monitor anywhere on the simulation domain. We place the time monitor in Fig. 2.8 just above the LED to measure the light output power. The monitor typically orients towards the top, as the side radiation radiates negligible fields compared to the top emitted radiation. The time monitor can be oriented and rotated in any manner

through the angular measures of phi and theta, if necessary. By RSoft convention, phi and theta measure in units of degrees, not radians.

Rsoft stores time monitors in two formats: $(1) *$.tmn files that contain the signed square root of the power output, $\mathrm{P}(\mathrm{t})$, and $(2) *$.ptm files that contain RSoft graph plotting files and also contain the actual power values of $\mathrm{P}(\mathrm{t})$. The power through a time monitor normally records instantaneous field measurements, although a time monitor can also output the average power over the last cycle of the simulation. In mathematical form, the instantaneous power in Eq. 2.6 derives from the normalized Poynting vector in the direction of the monitor: 


$$
S(t)=\frac{1}{S_{0}} \operatorname{Re}\left[\int_{A}\left[E(t) \times H^{*}(t)\right] \cdot d \vec{A}\right]
$$

where $\mathrm{S}_{0}$ is the power of the first launch field, used for normalization purposes. The differential area evaluates over the surface of the time monitor, the width and height parameters in Fig. 2.9 below, and orients by default in the positive z-direction (where phi and theta are 0$)$.

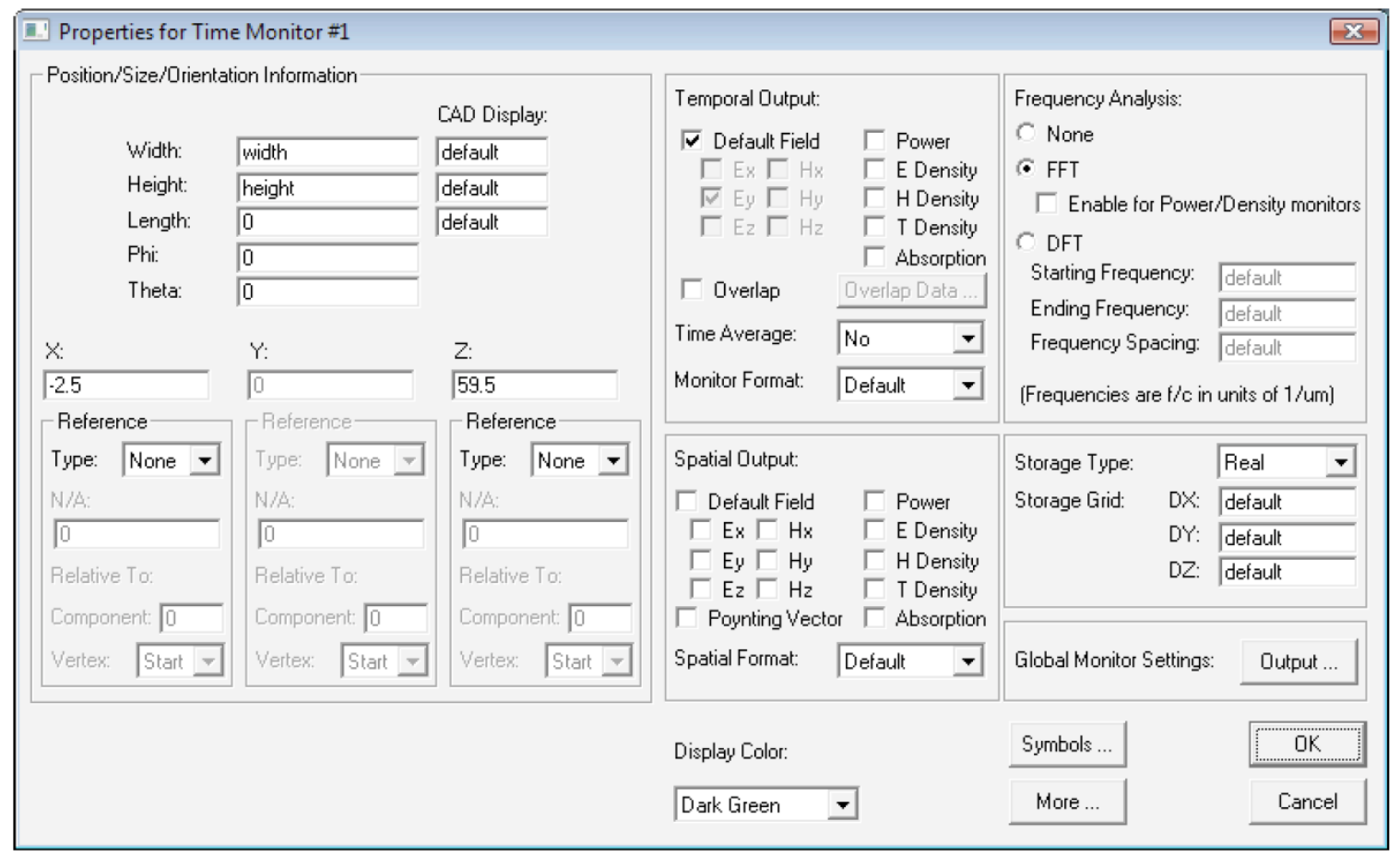

Fig. 2.9 - Time Monitor Window

\subsubsection{Stop, Monitor, and Update Times in RSoft FullWAVE'M}

Finally, the "Time Grid" titled border in Fig. 2.4 shows the stop, monitor, and update times. The stop time refers to how long the simulation runs before post-processing the time monitor data. The monitor time defines the time interval at which time monitors 
perform the calculation in Eq. 2.6 and stores the value in a .TMN file. The update time refers to the interval of time that the simulation domain updates visually for the user through the graphical user interface (GUI). This allows attentive users to spot deviating simulations and other problems with the simulation that may occur. Most often we only collect the data at the final time monitor value. Note that updating the screen at frequent intervals can slow down a simulation considerably, especially for large simulation domains and long simulation times, we often set this parameter to the a high value, such as " $65535 *$ fdtd_time_step" to effectively never update the screen during the course of the simulation but still collect monitor data at a regular interval set by the monitor time.

\subsection{Rsoft_tools Supplemental Software}

RSoft FullWAVETM robustly and thoroughly simulates the FDTD method but does not perform everything required by this thesis automatically. For example, data must endure post-processing, such as the averaging of the last few time monitor cycles to obtain the final field intensity. Also, we must generate many different grating types, such as the transmission grating, reflection grating, 3-fold symmetric photonic crystal grating, etc. Finally, multiple sources must be generated and randomly oriented to better represent incoherent light generation. This thesis author has written about a thousand lines of custom software that perform these exact tasks. Appendix B presents the code in its entirety. Note that the source code targets a Mac OSX system, but it should also perform equally well on any Unix-based OS. The author plans to port the application to Windows as the project's future work. 


\section{Chapter 3 - Single Reflection Grating GaN LED Simulations}

We now consider the first case of the single reflection grating or the bottom grating. The M-LLO procedure, described in the introduction, fabricates the reflection grating on a GaN LED. The LED mounts on a very thick layer of Si submount with an Ag reflector plate directly on top of it. The Ag fills the undoped $\mathrm{GaN}(\mathrm{u}-\mathrm{GaN})$ air holes in either a conical or cylindrical unit cell shape. Fig. 3.1 below details the LED model and shows an example conical grating structure.

\section{From Top to Bottom}

\section{Time Monitor p-GaN Quantum Wells $n$-GaN Undoped GaN Ag Reflector $\lambda=460 \mathrm{~nm}$}

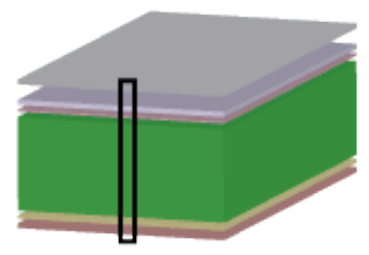

$\begin{array}{lll}\text { Material } & \text { Index of Refraction } & \text { Height (nm) } \\ p \text {-GaN } & 2.55 & 200 \\ \text { Quantum Wells } & 2.685 / 2.55 \text { (averaged) } & 67 \\ n \text {-GaN } & 2.55 & 4000 \\ \text { Undoped GaN } & 2.55 & 120+\mathrm{d} \\ \text { Ag Reflector } & \text { Linear dispersion terms } & 200+\mathrm{d}\end{array}$

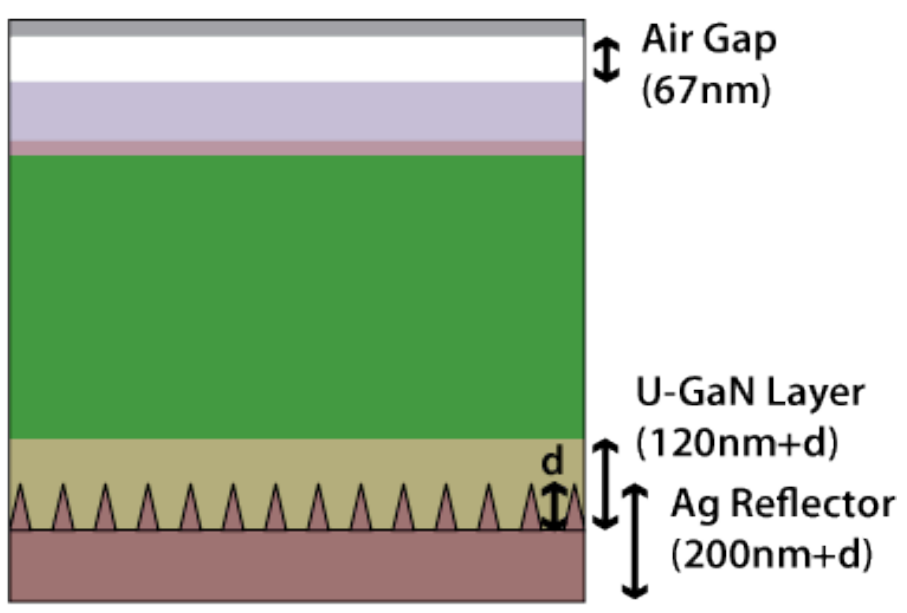
$(200 \mathrm{~nm}+\mathrm{d})$

Fig. 3.1 - Reflection Grating GaN LED Model

The FDTD method simulates and optimizes cases for many variable ranges over small incremental changes in feature size. The submount layer would add a large amount of 
calculation time; we did not include it in the simulation. It would be negligible effect on the light output of the LED, since the Ag reflector would reflect the light at a wavelength of 460nm. Put more positively, the reflective Ag layer strongly affects light output by reflecting light at the base of the thin-film LED. Finally, we place an n-type GaN (n$\mathrm{GaN}$ ) layer, multiple quantum well layers, and a p-type $\mathrm{GaN}(\mathrm{p}-\mathrm{GaN})$ layer on top of the grating and $\mathrm{u}-\mathrm{GaN}$ layer. The time monitor measures the time-varied light output of the whole device with a separation from the top of the LED of $67 \mathrm{~nm}$, a single grid size.

\subsection{Single Reflection Grating GaN LED Model}

Fig. 3.1 and Fig. 3.2 shows that cylindrical holes or conical holes have been fabricated on the bottom of the undoped GaN layer. The conical and cylindrical shapes represent $\mathrm{Ag}$ material, a reflective layer. Fig. 3.2(a) represents the conical grating model, while Fig. 3.2(b) represents the cylindrical grating model.

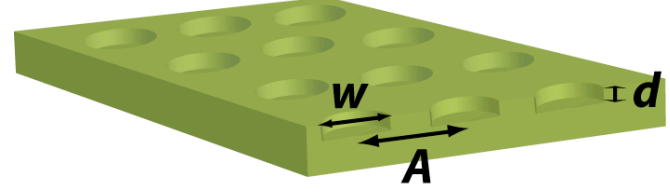

(a)

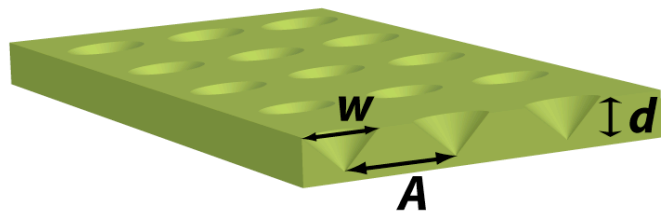

(b)

Fig. 3.2 - Reflection Grating Structures: (a) cylindrical and (b) conical

The yellow, solid models in Fig. 3.2 represent the u-GaN layer, later filled in by Ag, forming the reflective grating layer. Three parameters: grating cell period (A), grating cell height (d), and grating cell width (w), all shown visually in Fig. 3.2 and listed with description in Table 3.1, define a regular spacing between unit cells in a crystal lattice 
arrangement. The grating cell period, $A$, defines the center-to-center distance between unit cells. In 3-D, the parameter, $w$, represents a diameter, in the case of a circular structure (ie. sphere, cone, and cylinder), or a length of a side, in the case of a box structure (ie. cube, rectangular cube). The grating cell height, $d$, defines the depth of the bottom hole. These three parameters form the basis for all diffraction gratings in this thesis. The simulation method allows for fractional micron feature lengths, however we only use whole numbers. We define the parameter limits in the following way: $50 \leq \mathrm{d} \leq$ $200 \mathrm{~nm}, 1 \leq \mathrm{A} \leq 6 \mu \mathrm{m}, 1 \leq \mathrm{w} \leq 6 \mu \mathrm{m}, \mathrm{w} \leq \mathrm{A}$ to prevent overlap, and the smallest element must be larger than the smallest grid size. The fabricated grating height parameters range from $75 \mathrm{~nm}$ to $120 \mathrm{~nm}$ [18]. This determines our simulation range. Moreover, the minimum grid size limit determines the smallest $d$ value. If $\mathrm{d}<50 \mathrm{~nm}$, the simulation requires much smaller grids and longer simulation time. The width parameter cannot exceed the unit cell period since a larger value would indicate overlap of the cell structure, an invalid state. The smallest element must exceed the smallest grid size, as specified in the Yee's mesh simulation. If the grid cannot "see" the layer, then that element will be overlooked when calculating for the E and H fields using Maxwell's curl equations. This also determines the minimum distance from the power monitor to the device in Fig. 3.1, which we set to the minimum grid size, 67nm, above the LED.

We fixed the overall size of the FDTD simulation area to $100 \mu \mathrm{m} \times 100 \mu \mathrm{m}$. If $\mathrm{A}=1 \mu \mathrm{m}$, 100 unit cells can fit in the device. For $A=6 \mu \mathrm{m}$, only 16.67 unit cells can fit. Fig. 3.2 shows the grating as a square matrix of grating cells. We place the light source that emits constant waves at the center of quantum well region toward the top of the LED. 
Table 3.1 - GaN LED Model Simulation Parameters

\begin{tabular}{|lll|}
\hline Simulation & Descriptions & Simulated Ranges \\
\hline Parameters & Brief Description: & Range: \\
A & The period of the unit cells & 1 to 6 microns \\
w & The width of each unit cell & 1 to 6 microns \\
d & The height of each unit cell & 50 to $200 \mathrm{~nm}$ \\
\hline
\end{tabular}

The simulation starts from the moment the LED turned on from an off state. We must wait until the LED/monitor reaches a steady state for each grating simulation, after which we can extract the constant wave $(\mathrm{CW})$ average power. Each simulation sweep of the parameters produces a set of 20000 data points ( $2000 \mathrm{fs}$ at $0.1 \mathrm{fs} / \mathrm{step}$ ) at specific time periods during the simulation, and from this data set, we determined average power over the last cycle. In this range, maximum steady-state power radiates due to the $\mathrm{CW}$ source and reflections from the grating. We perform this procedure for each case.

\subsection{Single Reflection Grating GaN LED Model Simulation Results}

We simulate both conical and cylindrical reflection gratings to obtain the results below. We first simulate the conical reflection grating case and obtain the following results in Fig. 3.3, which show the results of a GaN conical-hole grating simulation, swept from $A=1 \mu \mathrm{m}$ to $A=6 \mu \mathrm{m}, \mathrm{w}=1 \mu \mathrm{m}$ to $\mathrm{w}=6 \mu \mathrm{m}$, and $\mathrm{d}=50 \mathrm{~nm}$ to $200 \mathrm{~nm}$. Similarly, Fig. 3.4 shows the results of a GaN cylindrical-hole grating simulation, sweeping over the same range. We indicate the flat plate (non-grating) results in each graph for comparison, 
represented by a solid line. We calculate average power over the last CW cycle of the simulation at a steady state value. In Fig. 3.3, we find that the conical-hole grating's maximum light extraction occurs at $A=1 \mu \mathrm{m}$ around the grating height $\mathrm{d}=200 \mathrm{~nm}$. The average power remains unremarkable until after $\sim 150 \mathrm{~nm}$, where average power rises dramatically. The $\mathrm{A}=2 \mu \mathrm{m}$ case peaks at $140 \mathrm{~nm}$, and clearly, the rest of the cases peak at around $158 \mathrm{~nm}$.

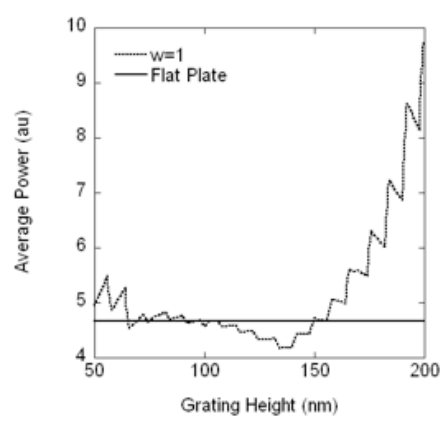

(a)

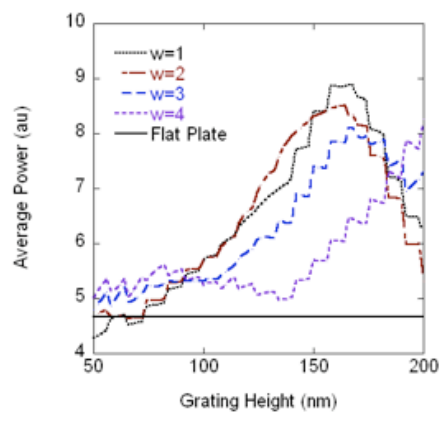

(d)

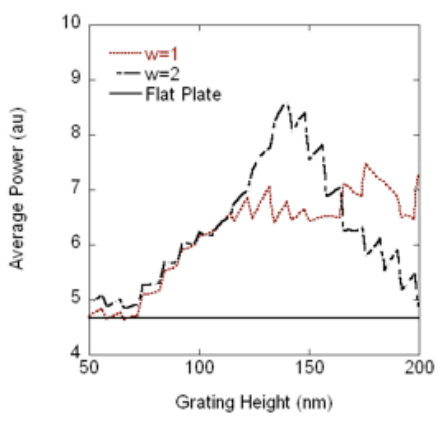

(b)

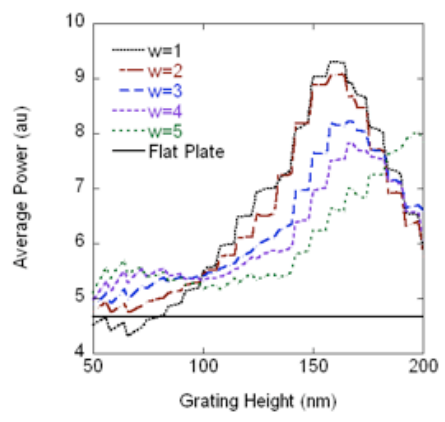

(e)

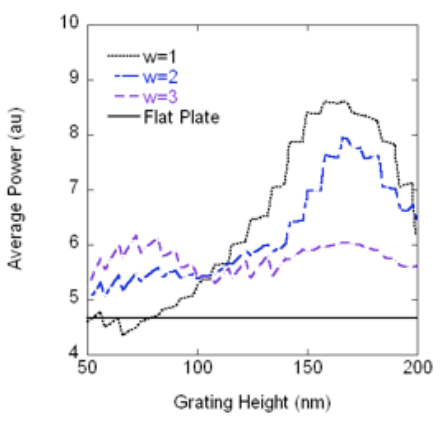

(c)

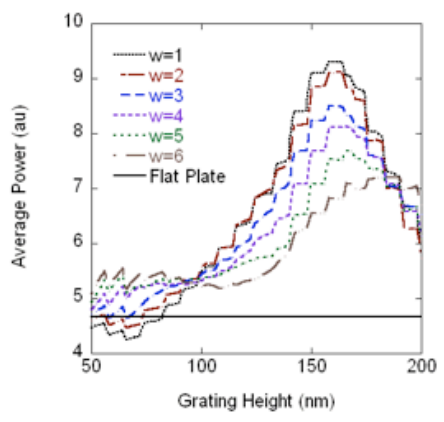

(f)

Fig. 3.3 - Average power for the conical reflection grating case: (a) $A=1$, (b) $A=2,(c) A=3,(d) A=4$, (e) $A=5$, and (f) $A=6 \mu \mathrm{m}$

For the same $A$ and $w$, the interface angle of the grating section varies as $d$ varies.

When $d=0$ or the flat case, the model does not improve. The interface angle increases with $d$ increasing, and so, we extract more light from the LED. Light extraction does 
depend on the grating depth, however, as evidenced by the cyclical pattern of the average power with the grating height. Therefore, the light emission efficiency drops at higher $d$ values. Light extraction efficiency maximizes for this particular structure at around $d=158 \mathrm{~nm}$. The best case in our simulation achieves a $112 \%$ light extraction improvement at a grating period of $A=6 \mu \mathrm{m}$, grating width $\mathrm{w}=1 \mu \mathrm{m}$, and grating height $\mathrm{d}=158 \mathrm{~nm}$. The grating width, $w$, for the conical-hole grating case affects the model in two ways. First, a greater $w$ increases the percentage of grating area compared to the total device area, the so-called "filling factor". If $w=0$, it does not affect light extraction improvement. When $w$ increases from zero, more light can be extracted. Also, we did not simulate cases where $w<1 \mu \mathrm{m}$ (nano-structure). Second, for the same $A$ and $d$, but varying $w$, the angle of the $\mathrm{Ag}$ and $\mathrm{GaN}$ interface also changes. When $w$ increases, the grating cell's angle decreases and shifts closer to the flat case. Therefore, at large $w$, the light extraction should decrease as $w$ increases. Summarizing the above two cases, a maximum $w$ design point exists for each grating; Fig. 3.5 shows this in detail. We did not simulate nano-scale gratings $(w<1 \mu \mathrm{m})$, only the micron-scale gratings. Based on the simulation results, the maximum grating design should be somewhere below $1 \mu \mathrm{m}$ (nano-structure). From the fabrication perspective, the fabrication capabilities of PKU at the time could not produce such nano-scale gratings. From the theoretical perspective, other factors should also be considered for the nano-grating design, such as the compatibility of such grating design with sub-wavelength, nano-scale feature sizes.

Finally, we notice that a single conical reflection grating may decrease light extraction efficiency for certain grating depths. But for most of the cases, single reflection gratings 
will improve light extraction. Each grating must be optimized to give the maximum light extraction efficiency for each type of single reflection grating.

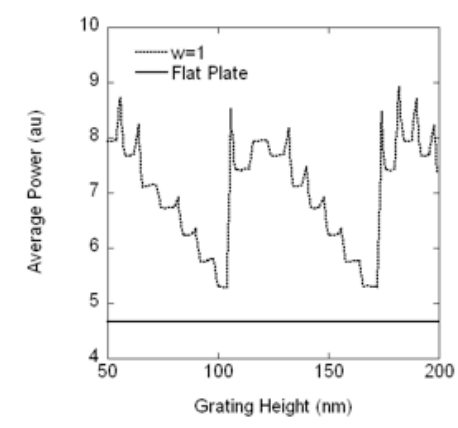

(a)

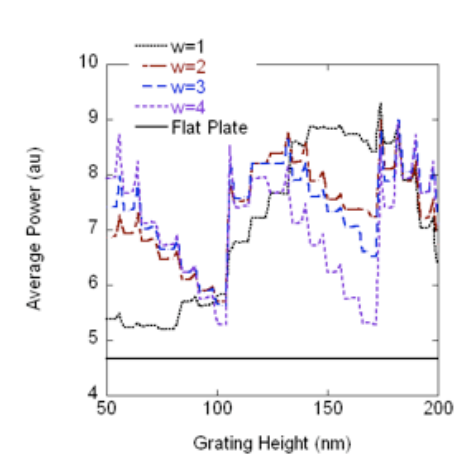

(d)

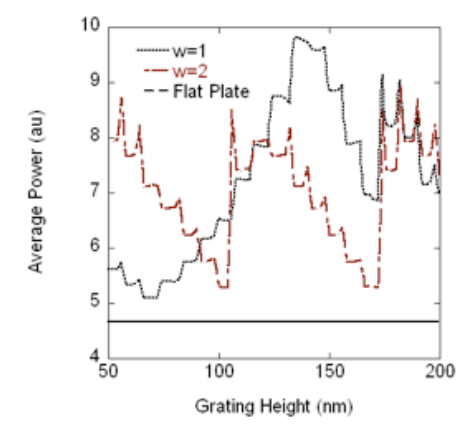

(b)

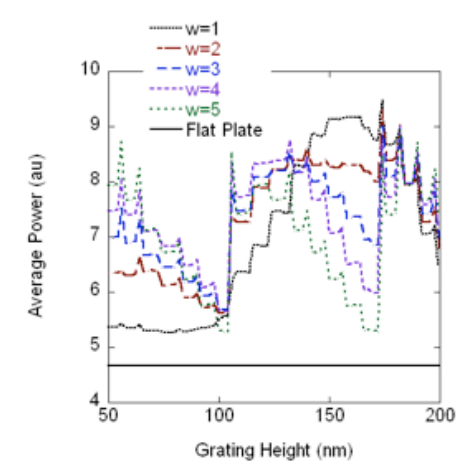

(e)

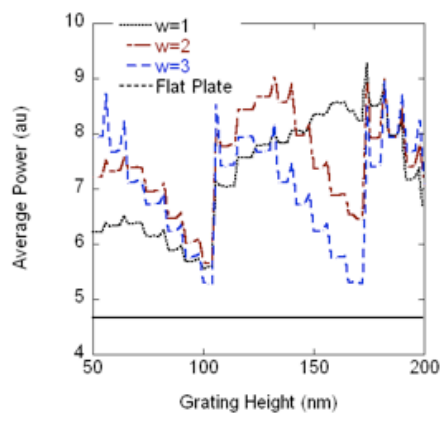

(c)

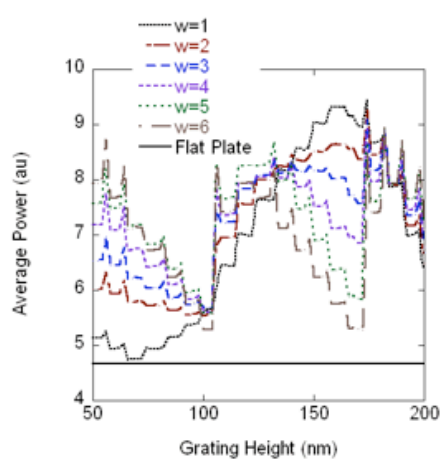

(f)

Fig. 3.4 - Average power for the cylindrical reflection grating case: (a) $A=1,(b) A=2,(c) A=3,(d)$ $A=4$, (e), $A=5$, and (f) $A=6 \mu m$

For the GaN cylindrical hole grating simulation shown in the Fig. 3.4, if the grating width, $w$, equals the grating period, then we observe very little light extraction improvement. But still similar to the GaN conical-hole grating simulation, a smaller $w$ produces the highest average power output. Two cases maximize average power output: the $A=2 \mu \mathrm{m}, w=1 \mu \mathrm{m}, d=136 \mathrm{~nm}$ case, which gives a light extraction improvement of $111 \%$, and the $\mathrm{A}=6 \mu \mathrm{m}, \mathrm{w}=1 \mu \mathrm{m}, \mathrm{d}=174 \mathrm{~nm}$, which gives a light extraction improvement of $102 \%$. The $w=A$ presents a very special case for the cylindrical grating structure, since 
the shape of the grating forms a 4-pointed star rather than four separated islands of GaN. This 4-pointed star structure consistently produces the worst case for light extraction efficiency among all of the grating period simulations.

Fig. 3.5 shows the summary of maximum power for the GaN conical- and cylindricalhole grating. In general, larger grating periods produce higher maximum power for both grating cases. This reveals a very important result to guide our fabrication and design. It implies that the micro-scale gratings prefer large-grating design, since the maximum power decreases with an increase of $w$. Compared to the conical-hole grating, the cylindrical grating achieves a maximum power at a slightly larger w value, due to the changing grating shape when $A=w$.

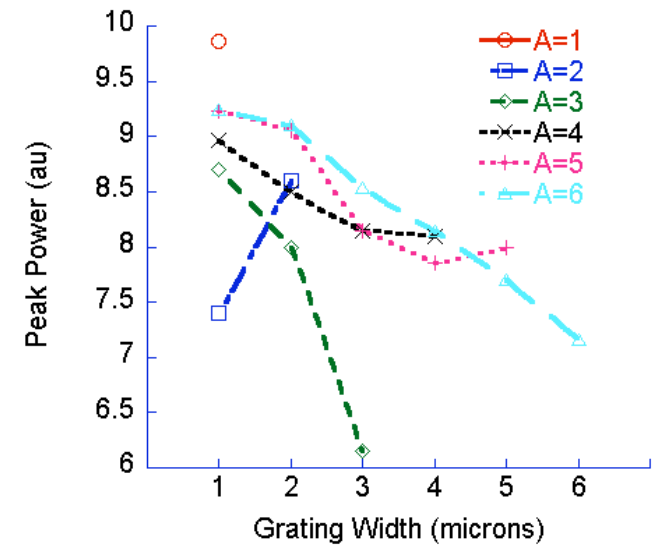

(a)

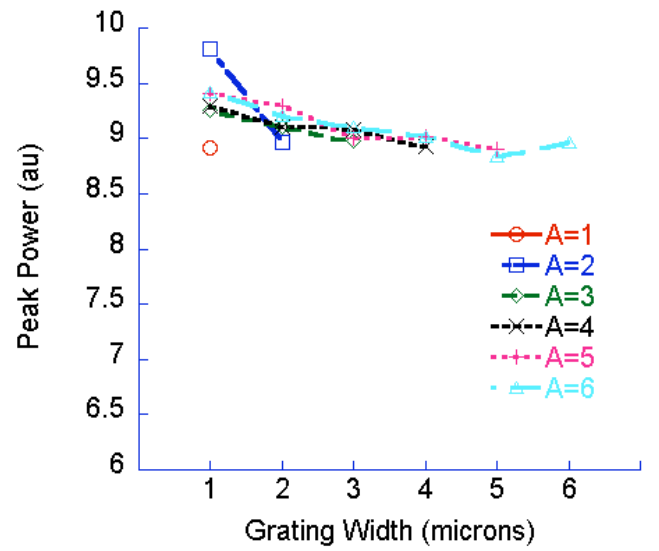

(b)

Fig. 3.5 - Peak Power vs. Grating Width for (a) conical and (b) cylindrical reflection gratings

\subsection{Single Reflection Grating GaN LED Results Summary and Conclusions}

We simulated a GaN LED with a single reflection grating using the FDTD method to calculate light extraction efficiency. Three grating parameters: $A, w$, and $d$ were studied. 
The cylindrical grating case achieves a $111 \%$ improvement of the light extraction, and the conical grating case achieves a $112 \%$ improvement compared to the non-grating case. The highest efficiency in a reflection grating resulted when $w=1 \mu \mathrm{m}$. As $w$ becomes small compared to $A$, the average output power increased. For both conical- or cylindrical-hole reflection gratings, the trend shows greater light extraction in designs where the reflection gratings at a larger grating period, (i.e. $A=6 \mu \mathrm{m}$ ), smaller grating width, (i.e. $\mathrm{w}=1 \mu \mathrm{m}$ ), and the grating height $\mathrm{d}$ around $174 \mathrm{~nm}$. Our simulation range did not reach the optimized $w$ value, which should have been less than $1 \mu \mathrm{m}$ in the nano-scale region. However, when simulating a grating with $w<1 \mu \mathrm{m}$, nano-grating characteristics should be addressed, since ray tracing methods could no longer describe grating effects accurately. 


\section{Chapter 4 - Top Transmission Grating GaN LED Simulations}

\subsection{Top Transmission Grating Background}

Now, we consider the top transmission grating's effects on GaN LED light extraction efficiency. Much research has already been completed on this subject, as the structure applies easily to fabrication, while reflection gratings present some difficulties in processing with the M-LLO procedure. We form the same grating cell shapes as the reflection gratings shown in Fig. 4.1, cylindrical and conical shaped grating cells.

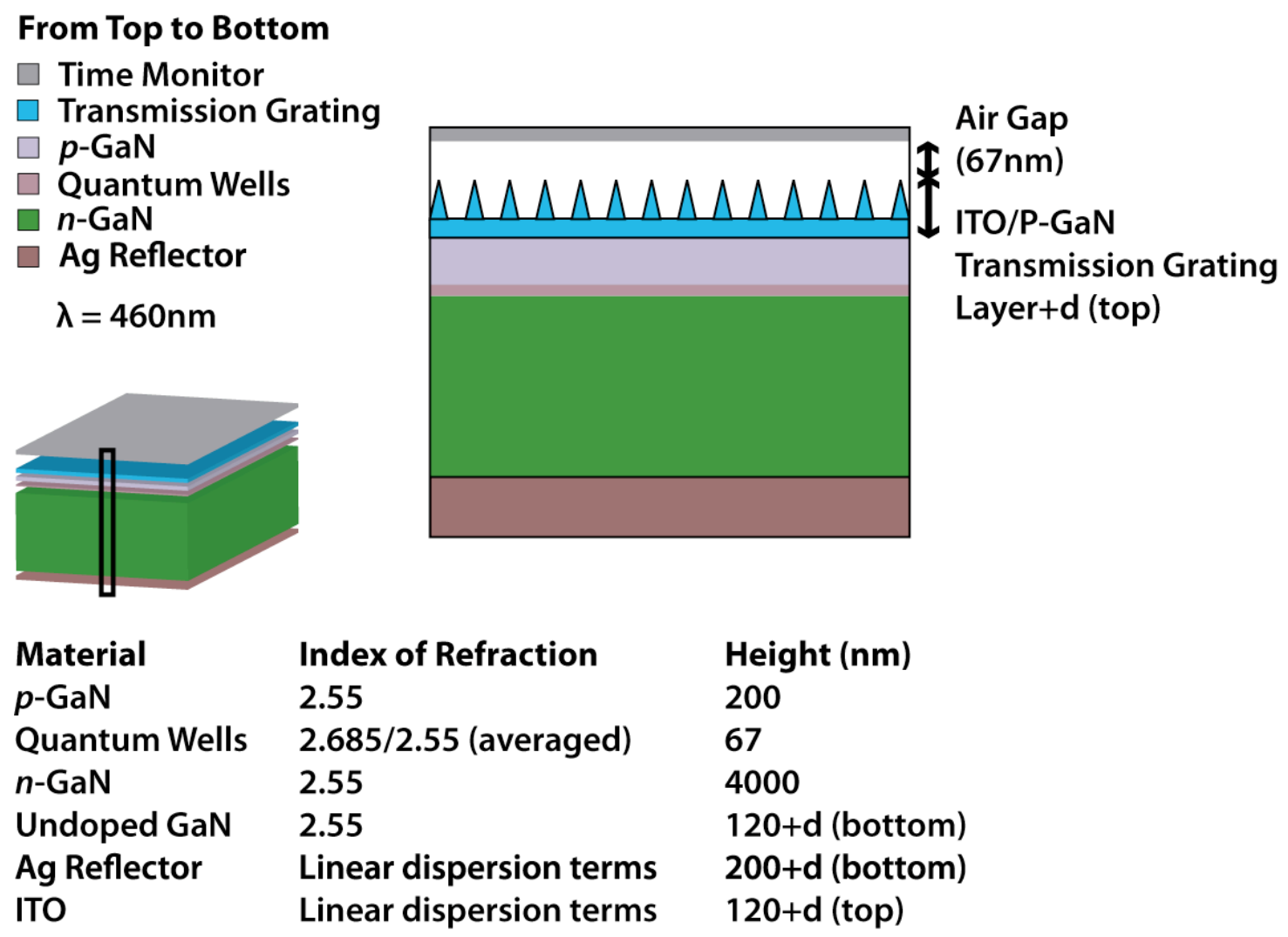

Fig. 4.1 - Transmission Grating GaN LED Model 
The transmission grating structure differs from the reflection grating structure, in that grating cells form pillars instead of holes. Also, since FDTD can freely define materials for any layer or shape, we choose three particular materials to represent our transmission grating: 1) non-lossy p-GaN, 2) lossy indium tin oxide (ITO), and 3) non-lossy ITO $(\alpha=0)$. The $p-G a N$ material encounters no loss because the loss factor, $\alpha$, is zero at the wavelength of $460 \mathrm{~nm}$. We define a regular spacing between unit cells in a crystal lattice arrangement by employing the following three parameters in Fig. 4.2, similar to the single reflection grating studies: grating cell period (A), grating cell height (d), and grating cell width (w). Fig. 4.2 shows the conical grating model in Fig. 4.2(a) and the cylindrical grating model in Fig. 4.2(b).

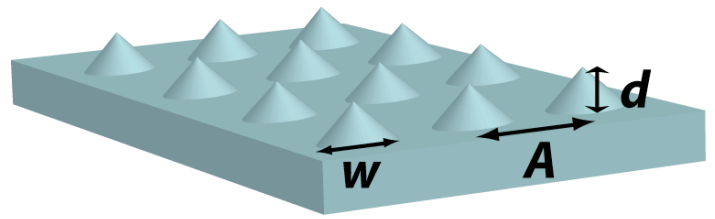

(a)

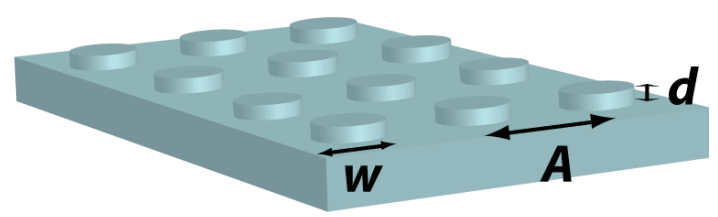

(b)

Fig. 4.2 - Transmission Grating Types: (a) conical and (b) cylindrical

\subsection{Single Top Transmission Grating GaN LED Simulation Results}

We obtain the results found in Fig. 4.3 to Fig. 4.10 by sweeping through the grating parameters, exactly like the reflection grating studies. Each case analyzes the significant findings in the following sections. 


\subsubsection{Non-Lossy p-GaN Transmission Grating Simulation Results}

Starting with the p-GaN transmission gratings, Fig. 4.3 and Fig. 4.4 present the conical and cylindrical p-GaN transmission grating simulation results. We find the conical p-GaN transmission gratings maximize light extraction efficiency in the range of $\mathrm{A}=1 \mu \mathrm{m}$ and $\mathrm{A}=2 \mu \mathrm{m}$, as shown in Fig. 4.3(a) and Fig. 4.3(b).

The graphs in Fig. 4.3(a) and Fig. 4.5(a) both reveal non-lossy p-GaN conical transmission gratings maximize average power at $A=1 \mu \mathrm{m}, \mathrm{w}=1 \mu \mathrm{m}, \mathrm{d}=200 \mathrm{~nm}$, which output a maximum light improvement of $165 \%$ over the non-grating case. Specifically, the peak power vs. grating height graph in Fig. 4.5(a) reveals a trend: when $A$ approaches the dimensions of $w$, light extraction efficiency generally increases. The grating appears largely unaffected by grating period or grating height until the transmission grating begins to reach feature sizes comparable to the wavelength of light. Again, this suggests that grating efficiency would be improved in the nano-scale, but in this case, the more transmission grating cells fabricated on the surface of an LED, the higher the light output. We will explore the topic of grating cell density and light extraction improvement more in Chapter 8 of this thesis, when we simulate grating structures with higher-ordered symmetries. 


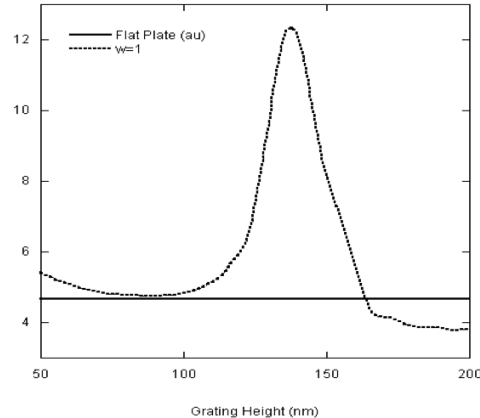

(a)

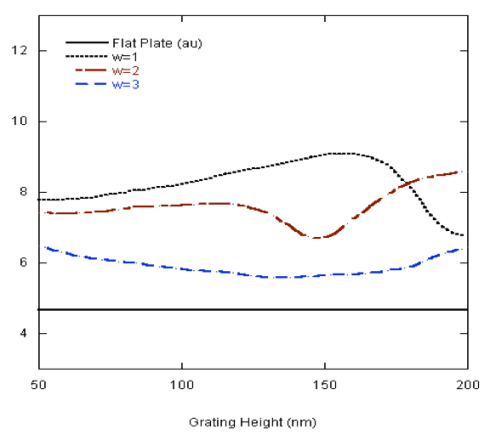

(c)

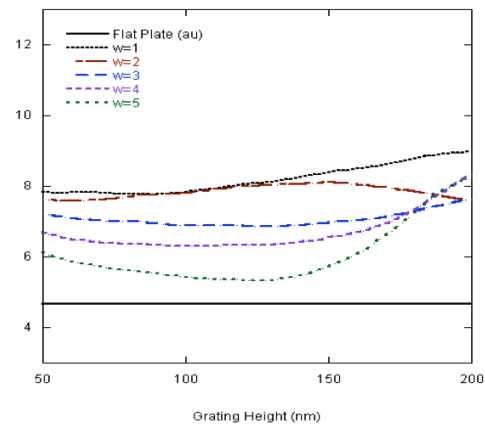

(e)

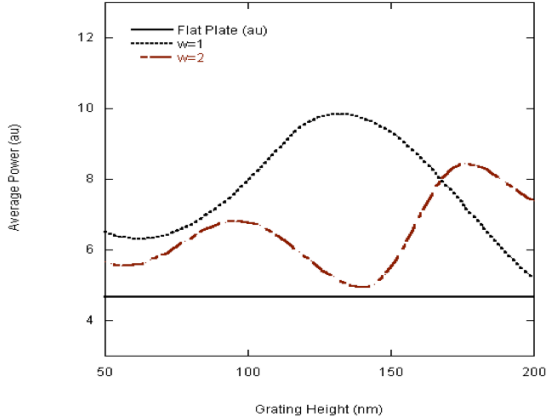

(b)

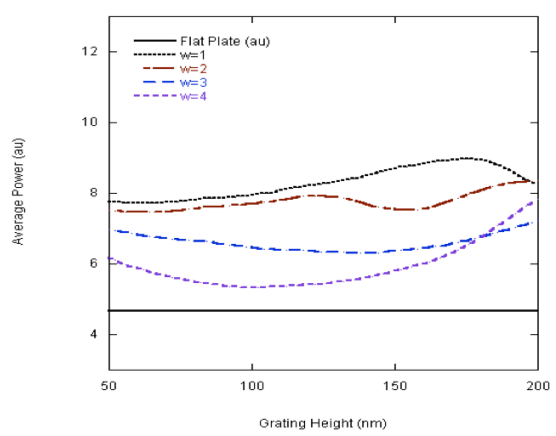

(d)

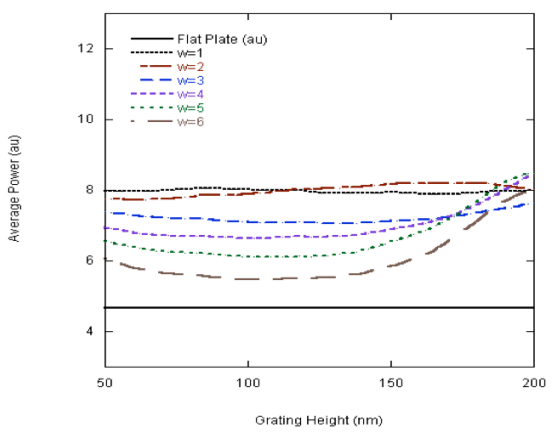

(f)

Fig. 4.3 - Average power for the conical non-lossy p-GaN transmission grating case: (a) $A=1$, (b) $A=2$, (c) $A=3$, (d) $A=4$, (e) $A=5$, and (f) $A=6 \mu \mathrm{m}$ 


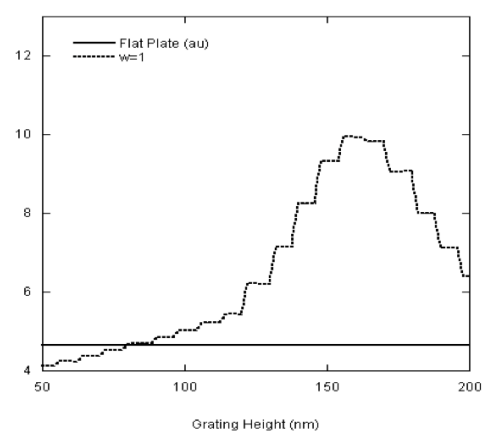

(a)

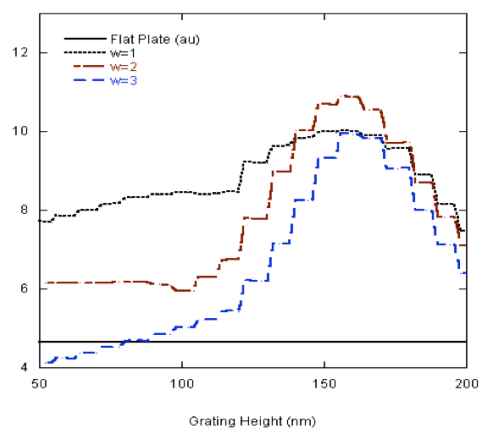

(c)

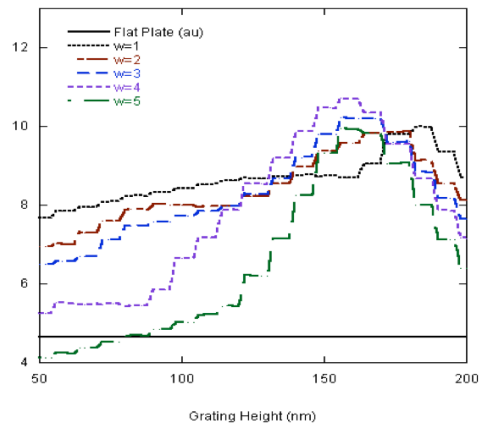

(e)

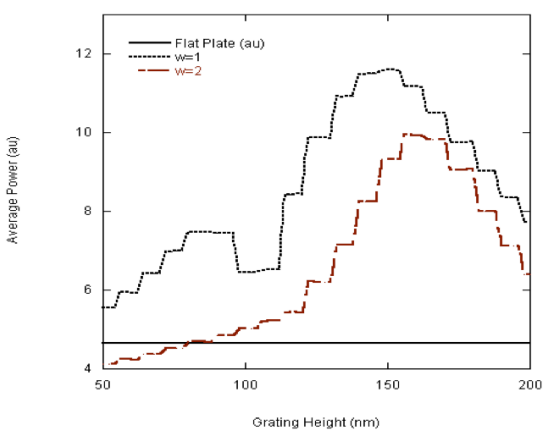

(b)

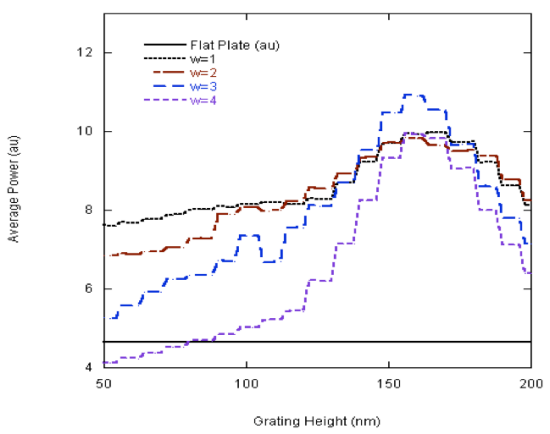

(d)

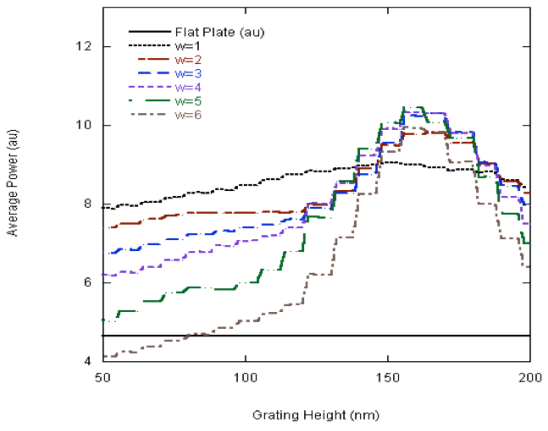

(f)

Fig. 4.4 - Average power for the cylindrical non-lossy p-GaN transmission grating case: (a) $A=1$, (b) $A=2$, (c) $A=3$, (d) $A=4$, (e) $A=5$, and (f) $A=6 \mu \mathrm{m}$ 
For non-lossy, $\mathrm{p}-\mathrm{GaN}$ cylindrical pillar transmission gratings, the $\mathrm{A}=\mathrm{w}$ case continues to produce results below the $\mathrm{A}>\mathrm{W}$ case. Again, due to the transformation of the basic shape of the grating unit cell from cylindrical pillars into 4-star shaped holes, an undesirable grating pattern in terms of light extraction improvement. Fig. 4.4 shows the full simulation set for cylindrical pillars. The peak power vs. grating height graph in Fig. 4.5(b) shows very clearly that each of the cases where $A=w$, decays into the same unfavorable four starred-hole shape, as in $\mathrm{A}=1 \mu \mathrm{m}, \mathrm{w}=1 \mu \mathrm{m}$. Otherwise, the optimal grating strikes a balance between maximizing $w$ with respect to $A$, maximizing the number of transmission grating cells ontop of the LED, and maintaining the cylindrical pillar grating structure. The non-lossy $\mathrm{p}-\mathrm{GaN}$ cylindrical pillar transmission grating case of $\mathrm{A}=2 \mu \mathrm{m}, \mathrm{w}=1 \mu \mathrm{m}$ maximizes light extraction efficiency by balancing all of these conditions.

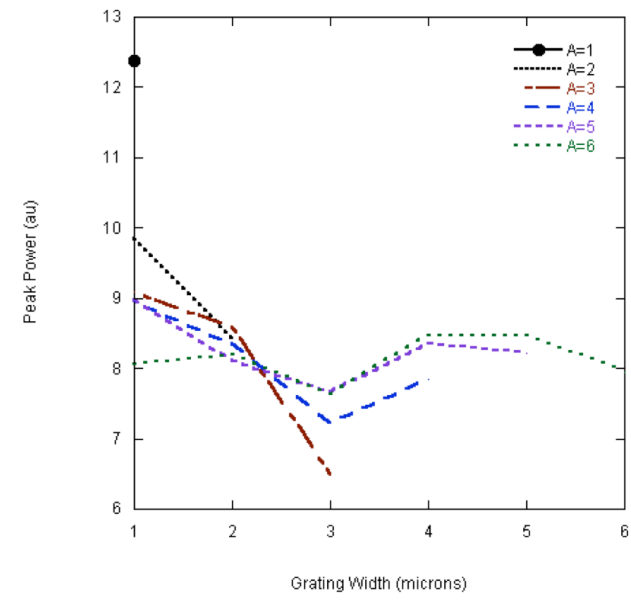

(a)

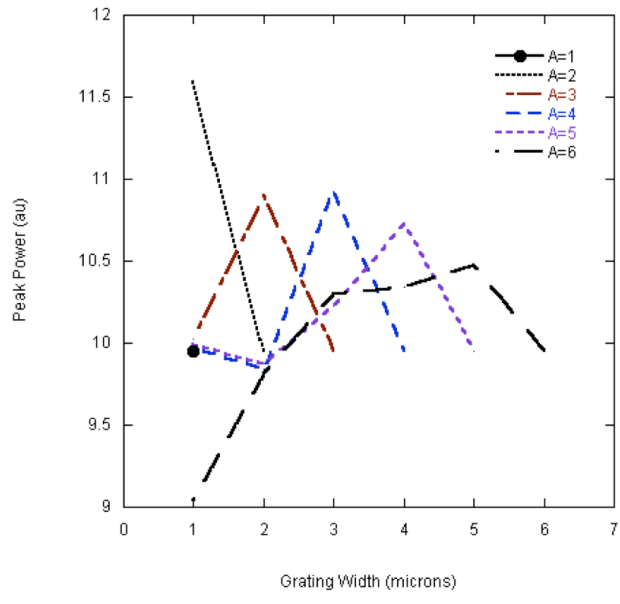

(b)

Fig. 4.5 - Peak Power vs. Grating Width for (a) conical and (b) cylindrical non-lossy p-GaN transmission gratings 


\subsubsection{Lossy ITO Transmission Grating Simulation Results}

Next, we simulated a lossy ITO transmission grating structure. Fig. 4.6 shows the full simulation set for the lossy conical ITO transmission grating. A grating period of 4,5 , and $6 \mu \mathrm{m}$ maximizes light extraction efficiency at approximately 8.4 au. Again, the peak power maximizes when $w$ equals $A$. The more grating cells that pack closely together, the greater the light extraction efficiency, maximizing at $A=w$. All transmission gratings in this simulation set increase light extraction efficiency. With the lossy conical ITO transmission grating, light extraction efficiency maximizes at about $80 \%$. At the very minimum, the lossy conical ITO transmission grating results in about a $30 \%$ increase in light extraction efficiency. Also, Fig. 4.7 shows the full simulation set for the lossy cylindrical ITO transmission grating. A grating height of $118 \mathrm{~nm}$ at $A=1 \mu \mathrm{m}$ and $w=1 \mu \mathrm{m}$, maximizes light extraction efficiency, resulting in a light extraction increase of $95 \%$ over the non-grating case. 


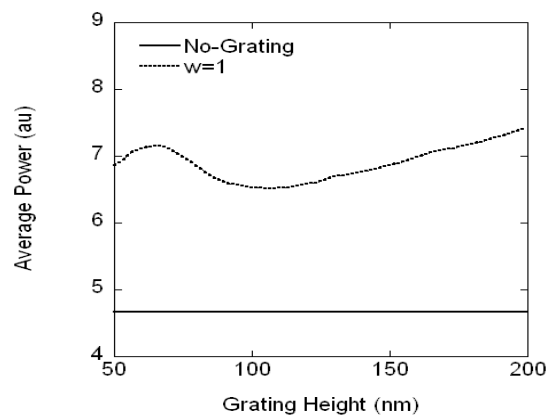

(a)

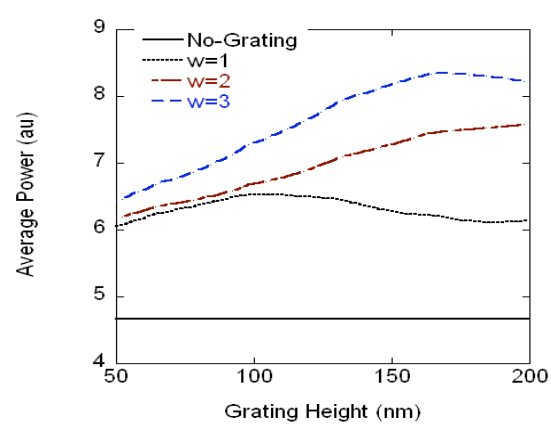

(c)

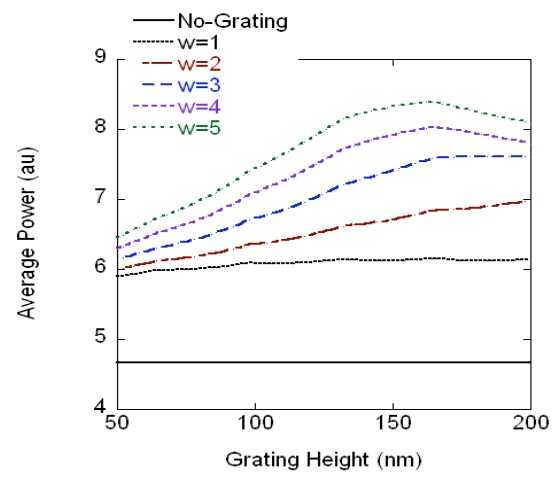

(e)

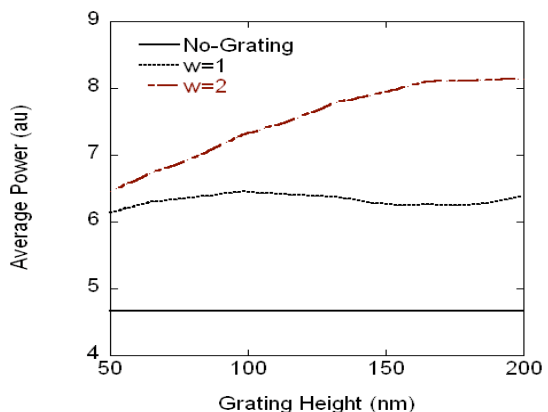

(b)

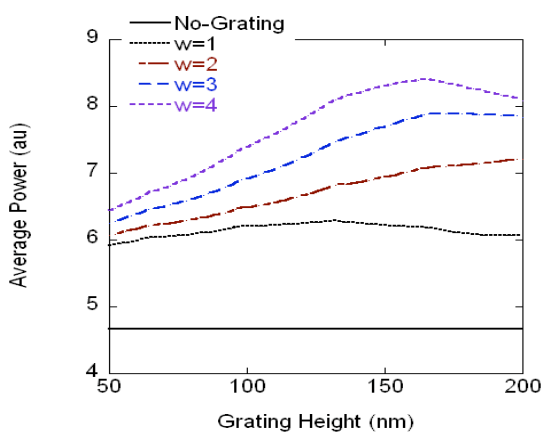

(d)

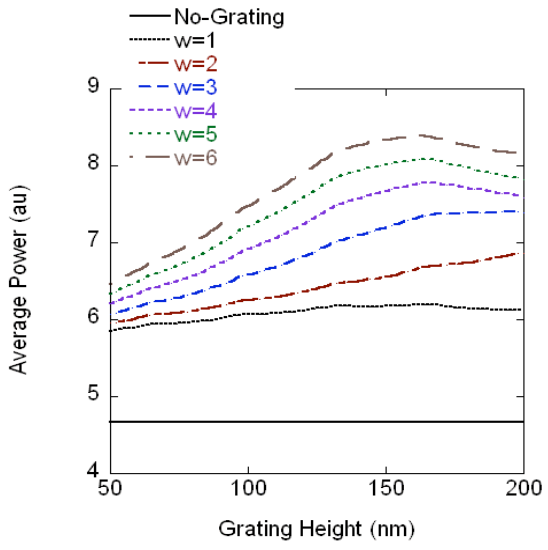

(f)

Fig. 4.6 - Average power for the lossy conical ITO transmission grating case:

(a) $A=1$, (b) $A=2$, (c) $A=3$, (d) $A=4$, (e) $A=5$, and (f) $A=6 \mu m$ 


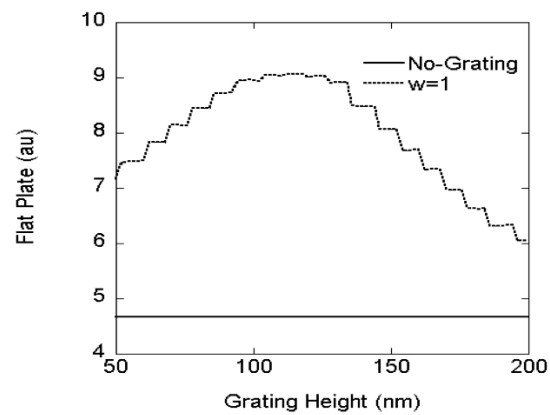

(a)

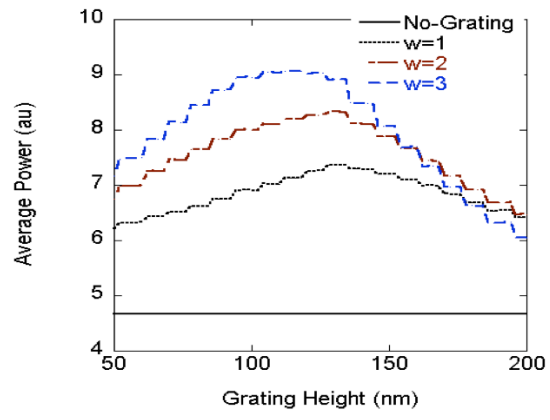

(c)

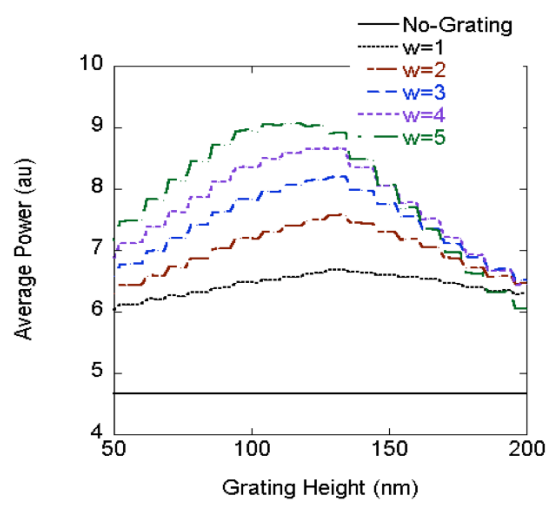

(e)

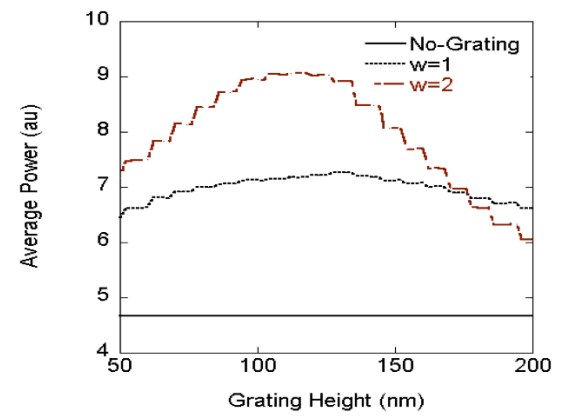

(b)

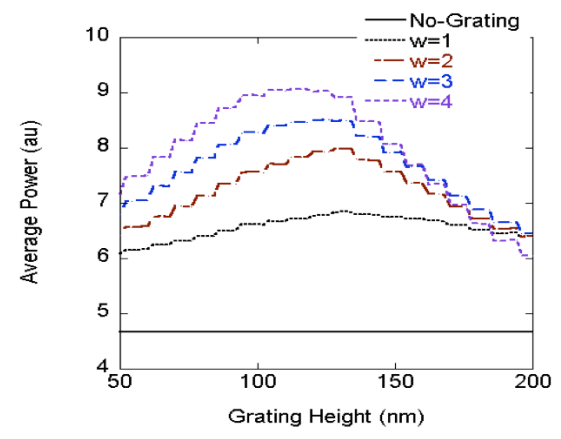

(d)

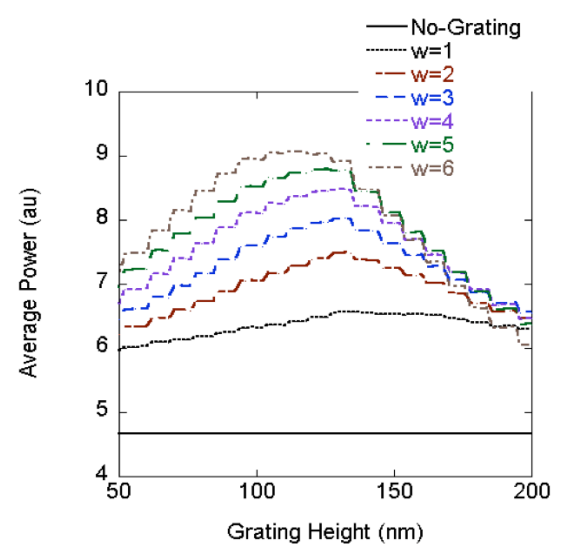

(f)

Fig. 4.7 - Average power for the lossy cylindrical ITO transmission grating case: (a) $A=1$, (b) $A=2$, (c) $A=3$, (d) $A=4$, (e) $A=5$, and (f) $A=6 \mu \mathrm{m}$ 


\subsubsection{Non-Lossy ITO Transmission Grating Simulation Results}

Finally, we simulated a non-lossy ITO transmission grating structure. Fig. 4.8 shows the full simulation set for non-lossy ITO conical pillar transmission gratings. The trend closely matches the lossy ITO structure but without the loss factors, resulting in a constant relative drop in output power between the two models. From Fig. 4.8 and 4.10(a), the structure optimizes at $A=4 \mu \mathrm{m}, \mathrm{w}=4 \mu \mathrm{m}, \mathrm{d}=166 \mathrm{~nm}$ with a $90 \%$ improvement. For the non-lossy ITO cylindrical pillar transmission grating, the results show similarities to the lossy ITO structure. From Fig. 4.9 and 4.10(b), the structure optimizes when $A=w$ and d=126 nm, with an improvement of $109 \%$ for all cases where $A=w$. 


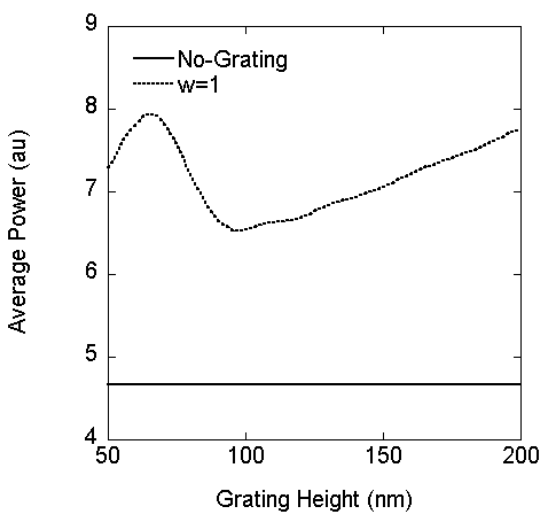

(a)

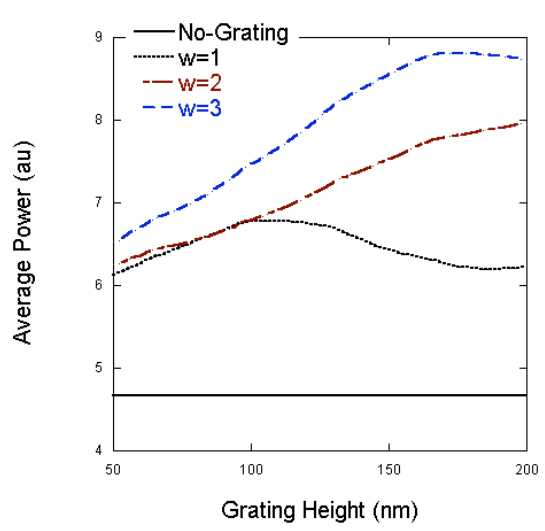

(c)

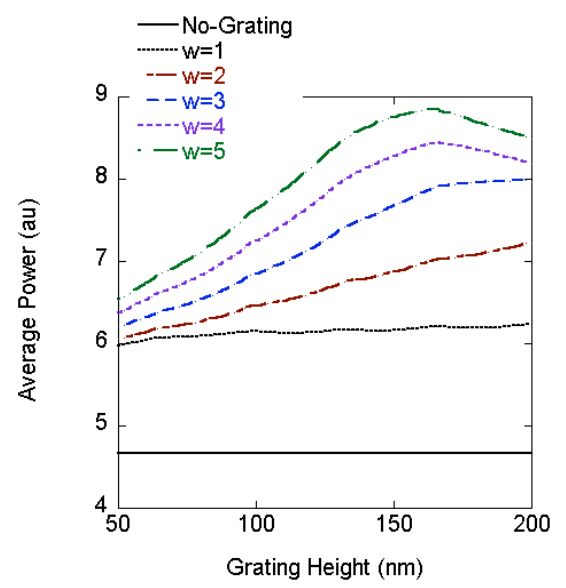

(e)

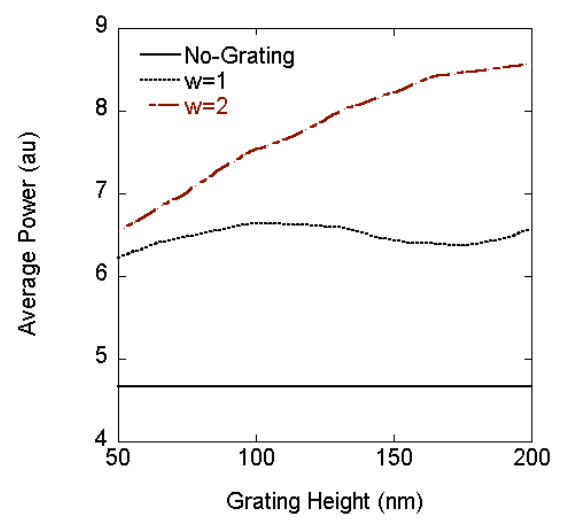

(b)

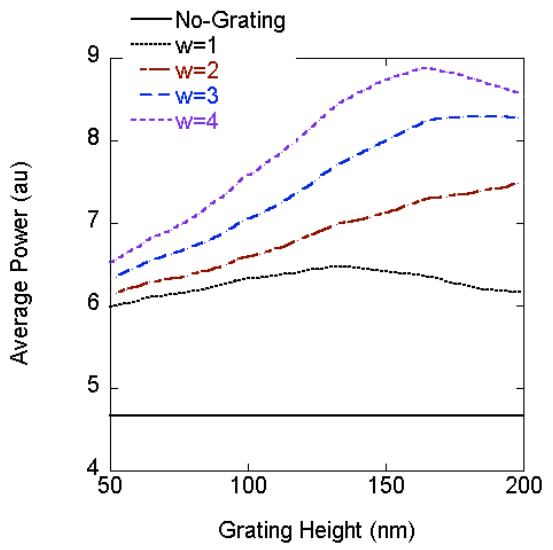

(d)

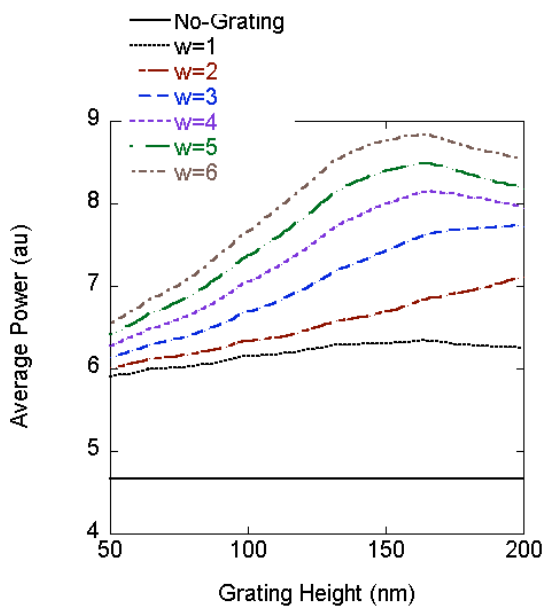

(f)

Fig. 4.8 - Average power for the non-lossy conical ITO transmission grating case: (a) $A=1$, (b) $A=2$, (c) $A=3$, (d) $A=4$, (e) $A=5$, and (f) $A=6 \mu \mathrm{m}$ 


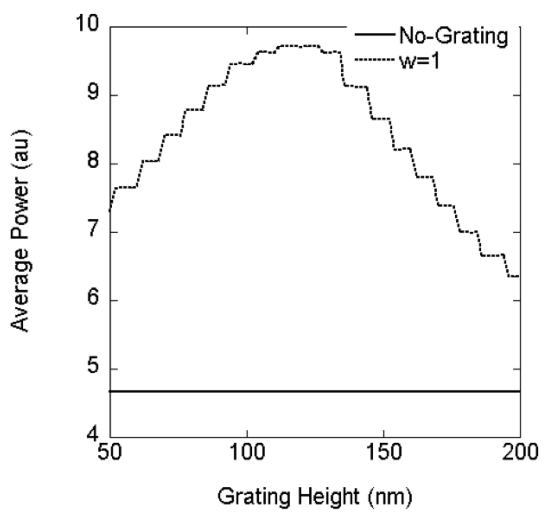

(a)

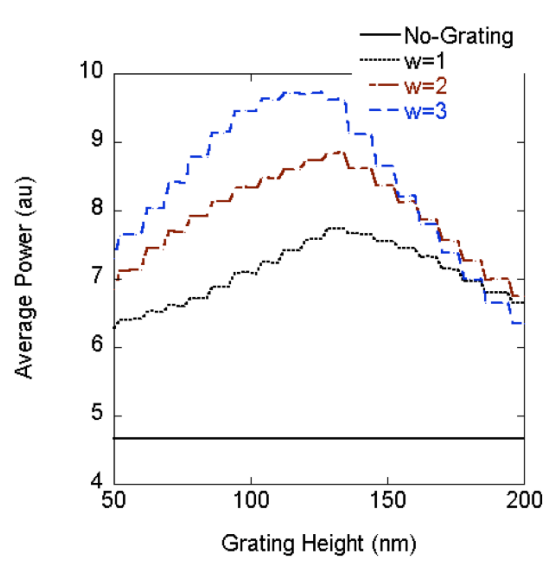

(c)

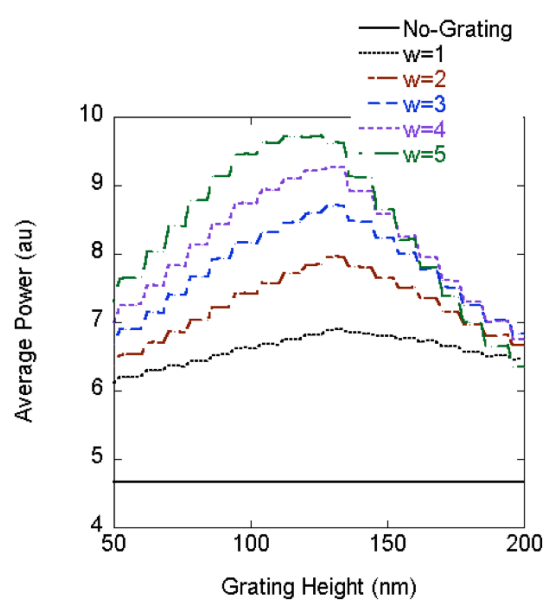

(e)

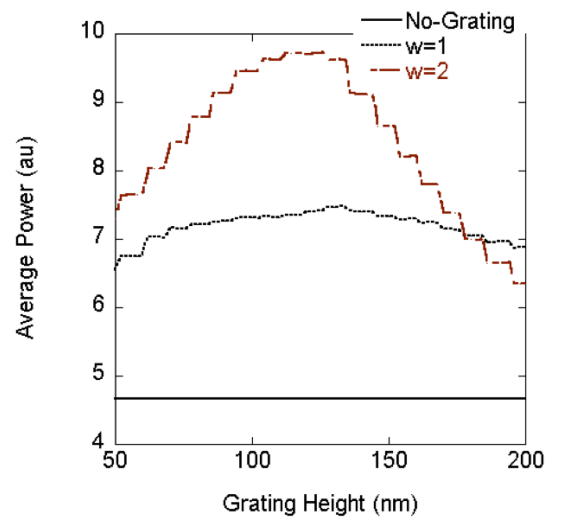

(b)

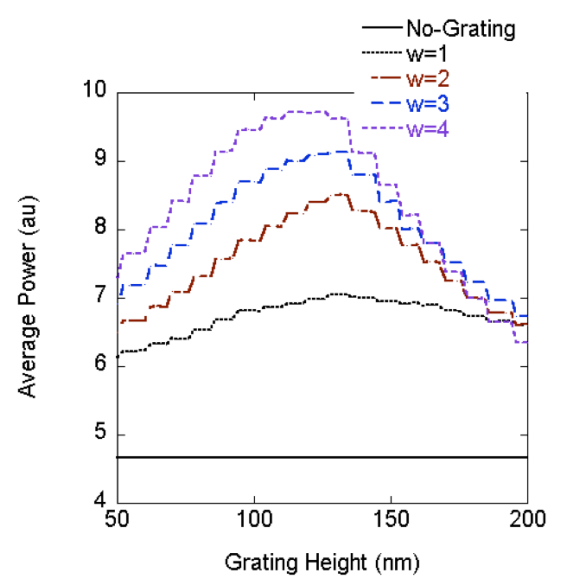

(d)

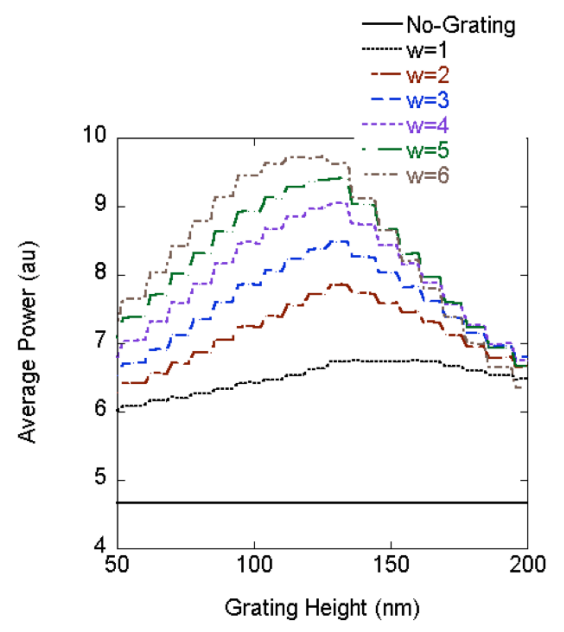

(f)

Fig. 4.9 - Average power for the non-lossy cylindrical ITO transmission grating case: (a) $A=1$, (b) $A=2$, (c) $A=3$, (d) $A=4$, (e) $A=5$, and (f) $A=6 \mu \mathrm{m}$ 


\subsection{Single Transmission Grating Results Summary and Conclusions}

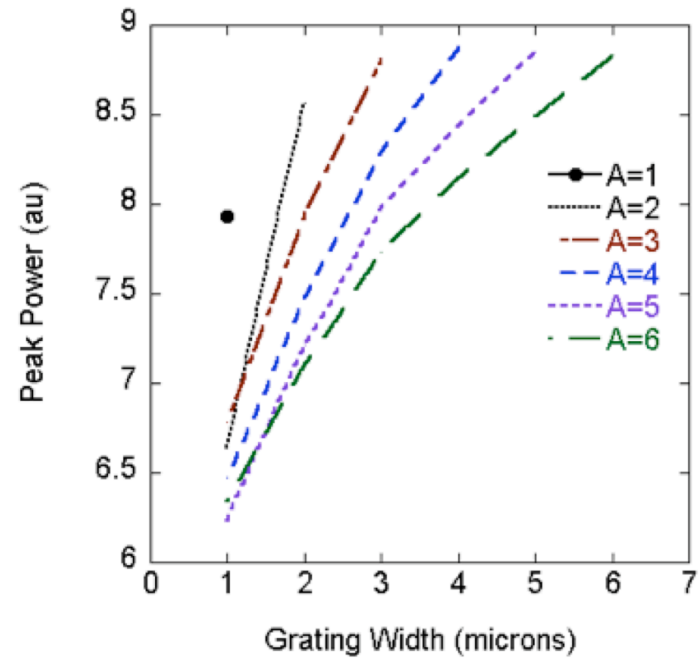

(a)

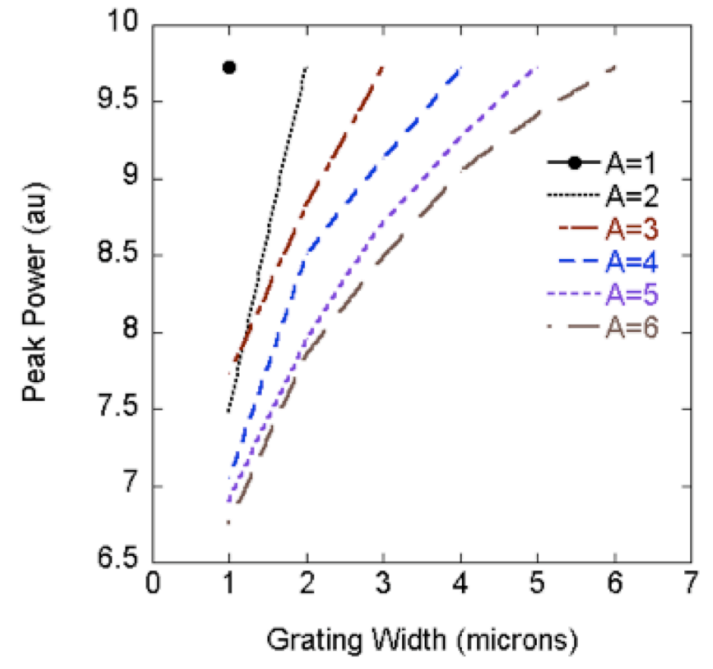

(b)

Fig. 4.10 - Peak power vs. grating width for non-lossy ITO transmission gratings: (a) conical and (b) cylindrical

Single transmission grating were simulated using the 2-D FDTD method. Three material types were simulated: non-lossy p-GaN, non-lossy ITO, and lossy ITO. In addition, a conical and cylindrical grating cell shape was simulated for each type of material. The results show that light extraction efficiency was maximized when using a non-lossy conical-shaped $\mathrm{p}-\mathrm{GaN}$ material for a transmission grating. The grating parameters for that particular grating were $A=1, w=1, d=138 \mathrm{~nm}$. Regardless of what grating type was used, light extraction efficiency improved by at least $30 \%$ over the reference case (no grating). This research has found what was already agreed in the literature, that grating structures influence light extraction efficiency in the following way: as $A$ compares similarly in dimension to $w$, light extraction efficiency improves. In 
other words, when grating cells closely pack, maximum light extraction efficiency should occur. This points our research towards a more closely packed structure, such as a 3 -fold symmetric photonic crystal structure with triangular symmetry and also smaller feature sizes in the nano-scale, such as the wavelength of light at $460 \mathrm{~nm}$, half-wavelengths, quarter wavelengths, etc. 


\section{Chapter 5 - Top and Bottom Grating GaN LED Simulations}

\subsection{Top and Bottom Grating Background}

The top transmission and bottom reflection grating simulation GaN LED simulations represent a novel concept in grating studies. Due to the high cost of fabrication, researchers in the field usually only study single gratings (ie. only one grating layer in the whole device). So, researchers typically do not simulate or experiment on top and bottom grating designs directly added together, much less the optimization of the two directly placed in the same device. Fig. 5.1 shows a top and bottom grating GaN LED with a bottom Ag reflector and top transmission grating.
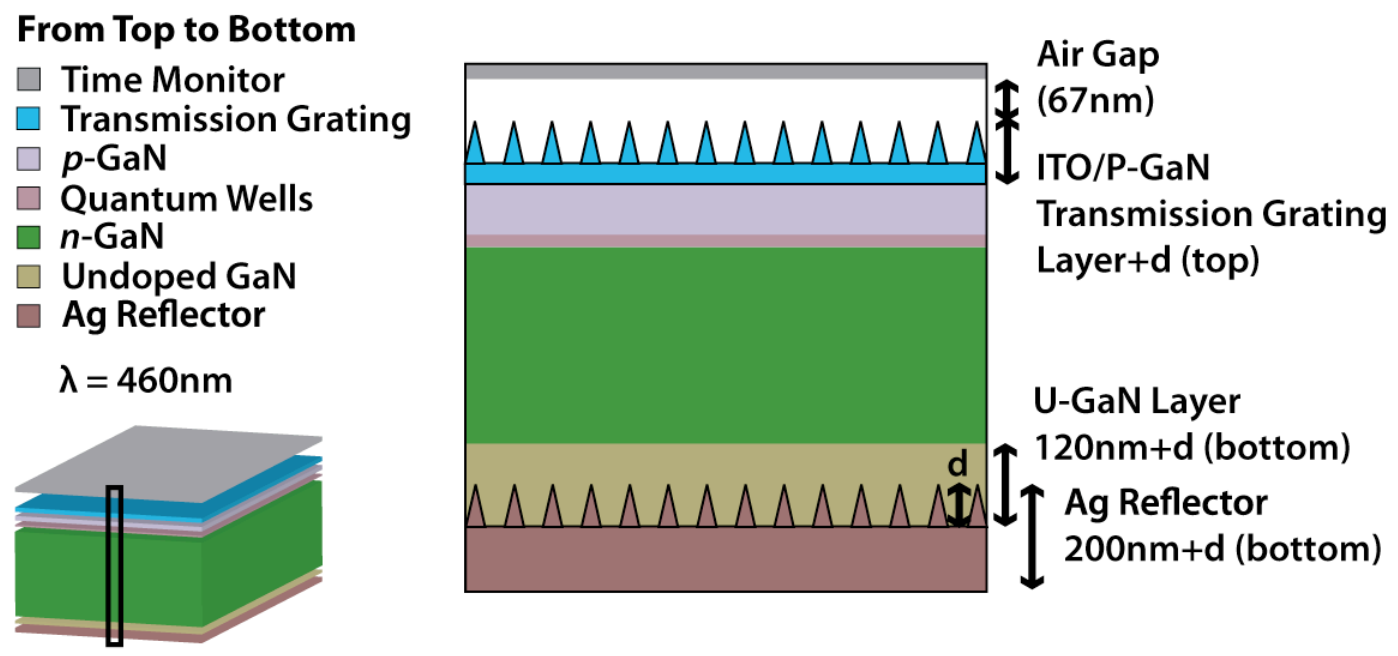

$\begin{array}{lll}\text { Material } & \text { Index of Refraction } & \text { Height (nm) } \\ p-G a N & 2.55 & 200 \\ \text { Quantum Wells } & 2.685 / 2.55 \text { (averaged) } & 67 \\ n \text {-GaN } & 2.55 & 4000 \\ \text { Undoped GaN } & 2.55 & 120+d \text { (bottom) } \\ \text { Ag Reflector } & \text { Linear dispersion terms } & 200+d \text { (bottom) } \\ \text { ITO } & \text { Linear dispersion terms } & 120+d \text { (top) }\end{array}$

Fig. 5.1 - Top and Bottom Grating GaN LED Model 
The following materials, shown in Table 5.1, compose the transmission gratings: a non-lossy conical P-GaN grating, a non-lossy cylindrical P-GaN grating, a non-lossy cylindrical ITO grating, a lossy cylindrical ITO grating, a non-lossy conical ITO grating, and a lossy conical ITO grating. The reflection gratings either use conical shapes or cylindrical shapes for the reflection grating. For each single grating case, we sweep the grating period, $A$, from 1 to $6 \mu \mathrm{m}$, the grating width, $w$, from 1 to $6 \mu \mathrm{m}$ (only for cases where $\mathrm{w}<\mathrm{A}$ ), and $d=50$ to $200 \mathrm{~nm}$. We have simulated these single grating cases and reported on them in the Chapters 3 and 4 of this thesis. We produce the optimized structure and list the results in the Table 5.1. The best cases for single gratings from each grating type were taken from our previous studies [16, 31], which also compare to and agree with experimental data provided by Peking University [9] [18]. Ref. [9] and [18] show the published experimental data on our simulated LED structure. Ref [9] only has electroluminescence (EL) data. Ref. [18] presents the experimental data of the modified laser lift-off (M-LLO) LEDs for the light extraction improvement. The experiment shows about $120 \%$ improvement for a $120 \mathrm{~nm}$-depth grating and $66 \%$ for a $75 \mathrm{~nm}$-depth grating [18], which agrees with our simulation. Our simulation gives a light extraction improvement of about $150 \%$ for the $120 \mathrm{~nm}$-depth grating and $65 \%$ for the $75 \mathrm{~nm}$-depth grating. Reference [16] gives a more detailed comparison of experimental data and simulation. Our simulation also shows that the single grating can achieve about 80 to $165 \%$ light extraction efficiency improvement. Table 5.1 presents a summary of optimized single grating structures. Therefore, the direct addition of two grating structures together in the same diode motivates the research in top and bottom grating 
structures.

Since each 2D simulation in FDTD takes approximately 10 minutes to simulate an LED model for a time up to $2000 \mathrm{fs}$, we must be mindful of the number of simulations performed in one simulation sweep. Top and bottom grating simulations produce many grating combinations that make it not possible to sweep through all of the possible combinations in the same way as the single gratings. The single gratings sweep across all valid cases of grating parameters (ie. A, w, and d), and this results in a total of 1,368 total cases. However, when performing the same operation for all combinations of both top and bottom gratings, the same simulation sweep requires a simulation of $1,871,424$

Table 5.1 - Summary of Optimized Single Grating Structures

\begin{tabular}{|c|l|c|c|c|c|c|}
\hline \multirow{2}{*}{$\begin{array}{c}\text { Transmission } \\
\text { (top) }\end{array}$} & Grating Type & $\begin{array}{c}\mathbf{A} \\
\mu m\end{array}$ & $\begin{array}{c}\text { W } \\
\mu m\end{array}$ & $\begin{array}{c}\text { D } \\
n m\end{array}$ & $\begin{array}{c}\text { Power } \\
\text { a.u. }\end{array}$ & $\begin{array}{c}\text { Increase } \\
\%\end{array}$ \\
\hline & $\begin{array}{l}\text { Conical } \\
\text { p-GaN }\end{array}$ & 1 & 1 & 138 & 12.374 & $165 \%$ \\
\cline { 2 - 6 } & $\begin{array}{l}\text { Non-Lossy } \\
\text { Cylindrical } \\
\text { p-GaN }\end{array}$ & 2 & 1 & 156 & 10.926 & $134 \%$ \\
\cline { 2 - 7 } & $\begin{array}{l}\text { Non-Lossy } \\
\text { Cylindrical ITO }\end{array}$ & 1 & 1 & 126 & 9.7258 & $109 \%$ \\
\cline { 2 - 7 } & $\begin{array}{l}\text { Lossy } \\
\text { Cylindrical ITO }\end{array}$ & 1 & 1 & 118 & 9.0752 & $95 \%$ \\
\cline { 2 - 7 } & $\begin{array}{l}\text { Non-Lossy } \\
\text { Conical ITO }\end{array}$ & 4 & 4 & 166 & 8.8755 & $90 \%$ \\
\cline { 2 - 7 } & $\begin{array}{l}\text { Lossy Conical } \\
\text { ITO }\end{array}$ & 4 & 4 & 164 & 8.4015 & $80 \%$ \\
\hline \multirow{7}{*}{$\begin{array}{c}\text { Reflection } \\
\text { (bottom) }\end{array}$} & Conical & 1 & 1 & 200 & 9.8650 & $112 \%$ \\
\cline { 2 - 7 } & Cylindrical & 2 & 1 & 136 & 9.8135 & $111 \%$ \\
\hline Reference & None & - & - & - & 4.6608 & 0 \\
\hline
\end{tabular}

grating cases. This clearly does not present a feasible solution at approximately 10 
minutes per simulation (the sweep would simulate for 35 years).

Based on the above information, an optimized top transmission grating pairs with an optimized bottom reflection grating to form a top and bottom grating, a total of 12 top and bottom grating combinations.

\subsection{Top and Bottom Grating GaN LED Simulation Results}

The single grating structures in combination with the top and bottom grating simulations account for over 181 grating cases, exploring the effect of adding two grating structures together directly in the same device. Appendix A shows each simulation result ranked by light extraction improvement. Table 5.2 lists the brief results of just the top and bottom grating simulations.

Table 5.2 - Top and Bottom Grating Simulation Results

\begin{tabular}{|c|l|c|c|c|c|c|c|c|c|c|}
\hline $\begin{array}{c}\text { Case } \\
\text { No. }\end{array}$ & \multicolumn{1}{|c|}{$\begin{array}{c}\text { Structure Name } \\
\text { Top Transmission }\end{array}$} & $\begin{array}{c}\mathbf{A} \\
\mu m\end{array}$ & $\begin{array}{c}\mathbf{w} \\
\mu \mathrm{m}\end{array}$ & $\begin{array}{c}\mathbf{d} \\
n m\end{array}$ & $\begin{array}{c}\text { Structure Name } \\
\text { Bottom Reflection }\end{array}$ & $\begin{array}{c}\mathbf{A} \\
\mu m\end{array}$ & $\begin{array}{c}\mathbf{w} \\
\mu m\end{array}$ & $\begin{array}{c}\mathbf{d} \\
n m\end{array}$ & $\begin{array}{c}\text { Power } \\
\text { a.u. }\end{array}$ & $\begin{array}{c}\text { Inc. } \\
\%\end{array}$ \\
\hline 1 & Lossy Conical ITO & 4 & 4 & 164 & Cylindrical & 2 & 1 & 136 & 6.6252 & 42 \\
\hline 2 & Non-Lossy Conical P-GaN & 1 & 1 & 138 & Cylindrical & 2 & 1 & 136 & 6.2106 & 33 \\
\hline 3 & Non-Lossy Conical ITO & 4 & 4 & 166 & Cylindrical & 2 & 1 & 136 & 6.1401 & 32 \\
\hline 4 & Non-Lossy Cylindrical P-GaN & 2 & 1 & 156 & Cylindrical & 2 & 1 & 136 & 6.1368 & 32 \\
\hline 5 & Non-Lossy Cylindrical ITO & 1 & 1 & 126 & Cylindrical & 2 & 1 & 136 & 5.5491 & 19 \\
\hline 6 & Lossy Cylindrical ITO & 1 & 1 & 118 & Cylindrical & 2 & 1 & 136 & 5.5181 & 18 \\
\hline 7 & Non-Lossy Conical ITO & 4 & 4 & 166 & Conical & 1 & 1 & 200 & 5.4614 & 17 \\
\hline 8 & Lossy Conical ITO & 4 & 4 & 164 & Conical & 1 & 1 & 200 & 5.3947 & 16 \\
\hline 9 & Non-Lossy Conical P-GaN & 1 & 1 & 138 & Conical & 1 & 1 & 200 & 5.2641 & 13 \\
\hline 10 & Non-Lossy Cylindrical P-GaN & 2 & 1 & 156 & Conical & 1 & 1 & 200 & 5.2185 & 12 \\
\hline 11 & Non-Lossy Cylindrical ITO & 1 & 1 & 126 & Conical & 1 & 1 & 200 & 5.0291 & 8 \\
\hline 12 & Lossy Cylindrical ITO & 1 & 1 & 118 & Conical & 1 & 1 & 200 & 4.8967 & 5 \\
\hline 13 & Non-grating (Reference) & - & - & - & None & - & - & - & 4.6608 & - \\
\hline 14 & Lossy Cylindrical ITO & 1 & 1 & 186 & Conical & 1 & 1 & 200 & 4.2404 & -9 \\
\hline 15 & Lossy Conical ITO & 4 & 4 & 52 & Cylindrical & 4 & 1 & 128 & 10.14 & 118 \\
\hline
\end{tabular}

The best-case top transmission gratings match with the best-case bottom reflection 
gratings to show effects of both together in percent increase with respect to the nongrating case. Table 5.2 lists cases $1-12$ as 12 combinations of 6 transmission gratings and 2 reflection gratings. These types of gratings significantly improve the non-grating case, or case 13 in Table 5.2. Cases 14 and 15 will be explained below with the destructive and constructive optical modes. The structure types from the rank ordered cases 1-12 show that a transmission grating structures prefer coupling with a cylindrical reflection grating, since ranks 1 to 6 all have a cylindrical reflection grating. Although conical reflection gratings sometimes perform better as a single grating case, this simulation reveals that optimal conditions for a single grating do not always apply for the top and bottom grating case. Also, conical transmission gratings perform better than cylindrical transmission gratings when combined with bottom reflection gratings. Conical structures dominate the top three ranks, and cylindrical transmission gratings dominate the following three rankings.

Since both the top and the bottom grating influence the optical field distribution in the non-grating LED and influence the light extraction, top and bottom gratings have coupling effects and need matching in design. They may or may not improve light extraction. In general, there exists a clear trend that shows greater light extraction efficiency favors conical transmission gratings and cylindrical reflection gratings. However, the results of the top and bottom gratings, derived from the best cases of reflection and transmission gratings only, perform more poorly (about $42 \%$ improvement maximum) than just the single grating structures alone. Trapped optical fields change with the addition of a grating structure, since the grating scattering depends on the grating 
structure parameters. The optimal conditions that produce the greatest light emission in a single grating case do not necessarily apply to the top and bottom grating case. When a reflection grating pairs with a transmission grating, the additional grating alters the optical fields within the device, therefore producing unfavorable light extraction due to the poor coupling or the mismatch between the gratings and the newly reorganized optical fields. In the worst case, a destructive interference or mismatch will degrade light extraction efficiency, as in Table 5.2, case 14. This last ranked structure even has worse light extraction efficiency (about $-9 \%$ ) than the non-grating case.

The best performing grating of the 12 combined top and bottom grating cases uses lossy conical-shaped ITO pillars as a transmission grating material matched with cylindrical-shaped $\mathrm{u}-\mathrm{GaN}$ holes as a reflection grating. This type of grating has a $42 \%$ improvement over the non-grating case. The research did not point to this result, since both lossy and non-lossy conical ITO transmission gratings ranked lowest in the transmission grating. The difference between the lossy and non-lossy materials is an imaginary loss factor in the index of refraction described by Eqs. 7(a) and 7(b):

$$
\begin{aligned}
& n_{\text {comp }}=n_{\text {real }}+j n_{\text {imag }} \text { and } \\
& n_{\text {imag }}=\frac{\gamma \lambda}{4 \pi},
\end{aligned}
$$

where $\gamma$ is the exponential loss factor in units of $\mathrm{um}^{-1}$ and $\lambda$ is the wavelength of light. Evanescent waves possibly penetrate the thin grating structure, causing greater light extraction efficiency. Also, the optical field for the cylindrical reflection grating may match better to the lossy transmission grating than the non-lossy transmission grating.

In the total rankings of all simulated cases, the best single transmission and the best 
single reflection grating combination ranks only at 158 out of 181 total cases simulated, or rank 9 in Table 5.2. Clearly, the direct addition of optimal grating structures does not linearly add to the total output power. The optical fields couple with an optimal set of grating parameters, which may be disturbed when we introduce a second grating. Another set of optimal grating conditions may exist, but it must be discovered by either a thorough sweep of all top and bottom grating parameters (6 parameters in total) or a combinatorial method that can isolate the absolute maximum in the simulation range. Double-grating design hinges critically on the optimization of both top and bottom gratings together. Clearly, the top and bottom gratings simulated here did not find the absolute maximum, or even relative maximum light extraction improvement, since none of them outperform the single grating cases alone.

Fig. 5.2(a) shows an example of the above case and explains the concept of mismatching. Here, we sweep the top grating height with the bottom grating height fixed and vice versa. The best top grating, non-lossy conical p-GaN transmission grating, optimizes with parameters of $\mathrm{A}=1 \mu \mathrm{m}, \mathrm{w}=1 \mu \mathrm{m}, \mathrm{d}=138 \mathrm{~nm}$. If we vary $d$ as a fabrication error from $118 \mathrm{~nm}$ to $186 \mathrm{~nm}$, then even slight variations result in, not only a non-optimal grating, but also the worst case. The best single bottom grating, conical reflection grating, optimizes at $\mathrm{A}=1 \mu \mathrm{m}, \mathrm{w}=1 \mu \mathrm{m}, \mathrm{d}=200 \mathrm{~nm}$ (a limit of our simulation). These two gratings totally mismatch with the optical fields. Therefore, when combining them, we only can achieve 13\% (5.26 a.u. power output, Case 9 in Table 5.2) of light extraction improvement, which falls short of the optimized single gratings. Fig. 5.2 also shows that the nano-level variation of depth can be a primarily influence of mismatching degree 
between optical fields. The experimental data published in Ref. [18] also supports the strong dependence of light extraction efficiency on the grating depth. Sequential single variable optimization simply obtains a local maximum. Therefore, we also perform some double grating optimization simulations based on Case 1 in Table 5.2 by varying one parameter at a time. We do not cover all the parameter's possible values, but we can still show the optimized double grating can be better than the single gratings. Fig. 5.2(b) and 5.2(c) show the results. We can achieve an 8.76 a.u. power output with the optimization of only the height value, which improves light extraction by $87 \%$. In addition, we also sweep $A_{\text {bottom }}$ and $w_{\text {bottom }}$ with fixed top grating parameters and fixed $d_{b o t t o m}$. The top lossy conical ITO grating at $A_{\text {top }}=w_{\text {top }}=4 \mu \mathrm{m}, d_{\text {top }}=52 \mathrm{~nm}$ and bottom cylindrical reflection grating at $A_{\text {bottom }}=4 \mu \mathrm{m}, w_{\text {bottom }}=1 \mu \mathrm{m}, d_{\text {bottom }}=136 \mathrm{~nm}$ produces a much more desirable power output of 9.76 a.u., about a $110 \%$ improvement. Finally, a further optimization with the same transmission grating but varying across $w_{\text {bottom }}$ and $d_{\text {bottom }}$ shows that $A_{\text {bottom }}=4 \mu \mathrm{m}, \mathrm{w}_{\text {bottom }}=1 \mu \mathrm{m}, d_{\text {bottom }}=128 \mathrm{~nm}$ gives an improvement of $118 \%$, as shown in Fig. 5.2(c). This result makes an educated guess at the absolute maximum might be but can still only be considered as a local maximum. An absolute maximum should be found from a thorough sweep of all top and bottom grating parameters' possible values.

However, the partially optimized double grating structure still outperforms the optimized single grating case, an $80 \%$ improvement for this type of single top grating and $111 \%$ improvement for this type of single bottom grating. All these calculations show that the optimization of a double grating requires more care at this critical step than a single grating structure. 


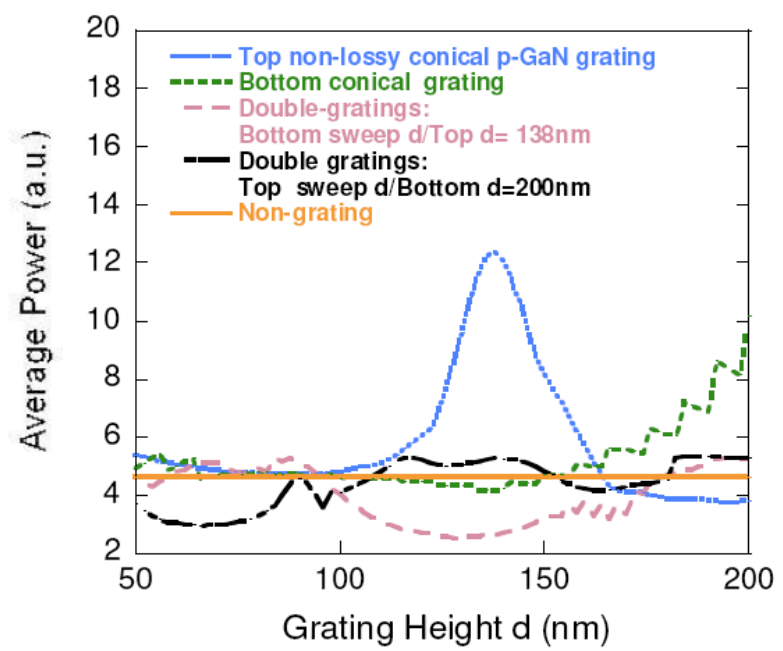

(a)

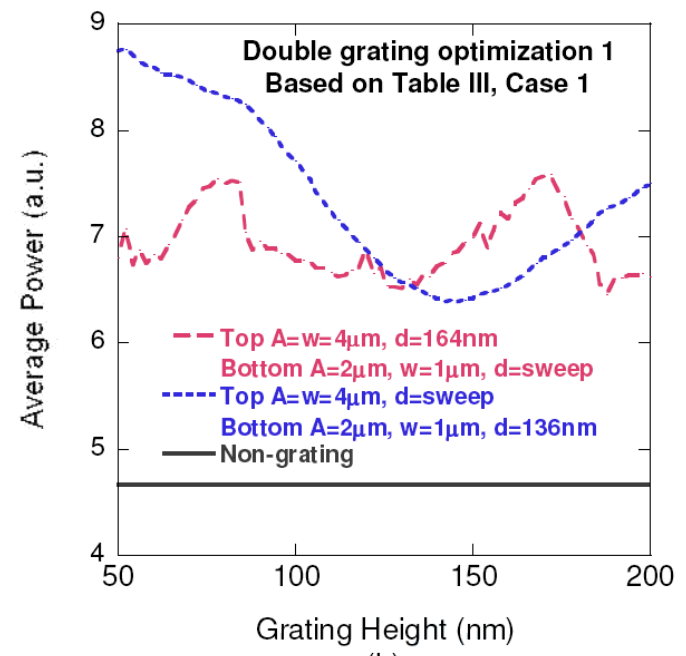

(b)

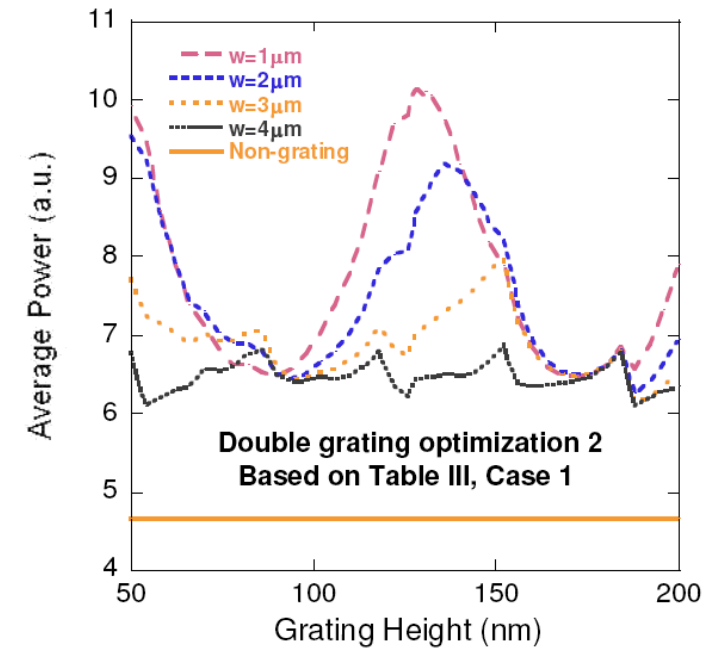

(c)

Fig. 5.2 - Partial Optimization Results: (a) comparison of gratings with only top, only bottom, and double gratings based on Table 5.2 Case 9 (Both top and bottom grating A=1mm, w=1mm), (b) double grating optimization 1 based on Table 5.2, Case 1, sweeping either bottom grating $d$ or top grating $d$, (c) double grating optimization 2 based on Table 5.2, Case 1, but with the top grating $A=w=4 \mu m, d=52 \mathrm{~nm}$ and bottom grating $A=4 \mu \mathrm{m}$, while sweeping the $w$ and d parameters 


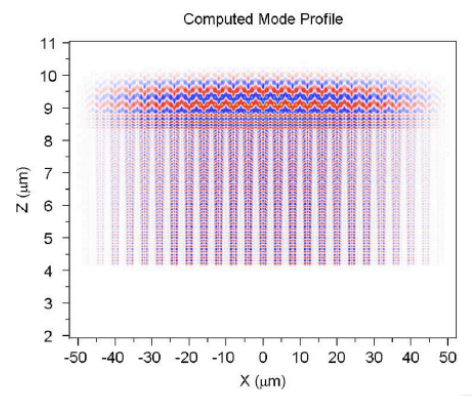

(a)

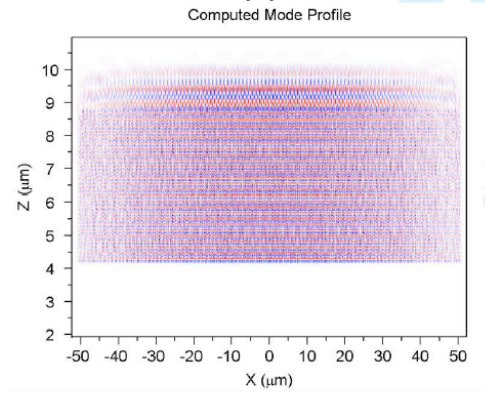

(d)

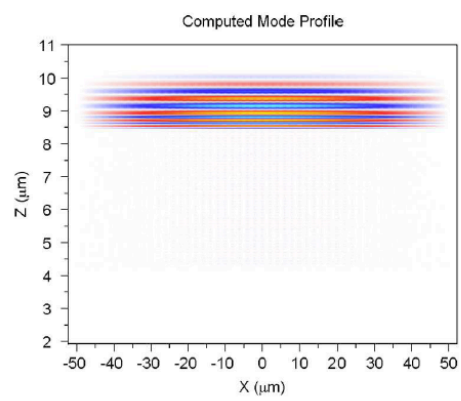

(b)

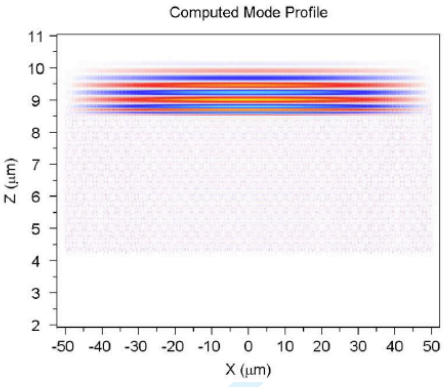

(e)

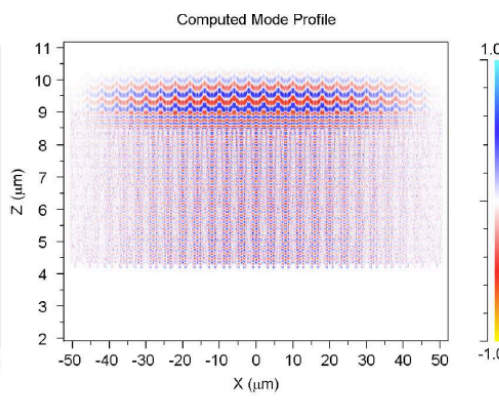

(c)

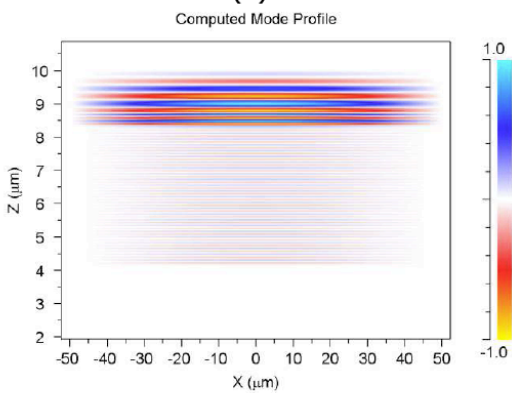

(f)

Fig. 5.3 - Mode profile of the following grating structures: (a) Top conical grating only $A=4 \mu m, w=4 \mu m$, $d=164 \mathrm{~nm}$, (b) Bottom cylindrical grating only $A=2 \mu \mathrm{m}, w=1 \mu \mathrm{m}, d=136 \mathrm{~nm}$, (c) Top and bottom grating (a)+(b), Table 5.2 Case 1. (d) Best top grating: conical $A=1 \mu \mathrm{m}, w=1 \mu \mathrm{m}, d=138 \mathrm{~nm}$ (Table 5.1), (e) Best bottom grating: conical $A=1 \mu \mathrm{m}, w=1 \mu \mathrm{m}, d=200 \mathrm{~nm}$ (Table 5.1), and (f)Nongrating LED case.

To further prove the field variation theory, we output the optical field distribution of

GaN LEDs. First, the optical field distributions in the top grating only in Fig. 5.3(a) and bottom grating only in Fig. 5.3(b) show stark differences between each other. For both single grating mode profiles, a strong optical field still exists within the MQW region and the p-GaN layer. When the top grating and bottom grating add together as in Table 5.2, Case 1, we arrive at the optical mode distribution shown in Fig. 5.3(c), the optical field distribution, and ultimate light extraction efficiency, will not prefer either top grating or bottom grating. An optical field cancellation effect (rather than enhancement effect) gives 
a light extraction efficiency of $42 \%$, which reveals a deficiency compared to single grating cases. Second, Fig. 5.3(d) and (e) presents the best single grating top- and bottomgrating optical field distributions. Compared to Fig. 5.3(a) and (b), the optical field of Fig. 5.3(d) and (e) escapes more from the active region. The optical field distribution varies more between Fig. 5.3(a) and (d), compared to that of Fig. 5.3(b) and (e). Also, the light extraction improvement differs more between Fig. 5.3(a) at 80\% and Fig. 5.3(d) at 165\%, compared to that of Fig. 5.3(b) at $111 \%$ and Fig. 5.3(e) at 117\%, as well. Finally, we present the non-grating LED case in Fig. 5.3(f), which has the strongest field around the active region compared to all other cases. In general, our simulation shows that better light extraction allows more optical fields to escape the active region. The top grating optical field and bottom grating optical field in our simulation may not matched, which presents very interesting results. The exact correlation of the double grating design, optical field, and light extraction efficiency requires intensive simulation, which travels outside the scope of this thesis.

The best single grating surprisingly does not relate much to the best double grating. This result presents many uses for most people in the LED research field, since many perform experiments only on single gratings. Designing a double grating with only optimized single gratings will only complicate the fabrication procedure. Due to the grating mismatch, the LED performance will not gain much light extraction efficiency, since the effects of grating structures do not necessarily add linearly to light extraction improvement. Detailed studies must be carried out before fabrication to determine maximum coupling efficiency, according to the optical field variation when adding an 
additional grating structure. Therefore, grating optimization procedures constitute a critical aspect of double-grating design. As long as one can match grating parameters to the optical field, the choice of grating parameters matters very little; the design can be chosen from what fabricates more conveniently. Therefore, the possibilities of grating design open up in this sense.

\subsection{Top and Bottom Grating GaN LED Results Summary and Conclusions}

We simulate top and bottom grating structures, taken from the optimized cases of transmission and reflection gratings alone, by the FDTD method. In general, grating structures will improve light extraction of LEDs compared to the non-grating structure. The simulation results also show that the introduction of a second grating significantly alters the original, optimized single grating optical fields. The partially optimized double grating can achieve a light extraction improvement of $118 \%$ with respect to the nongrating case, showing that a double grating structure can outperform the single grating structures performing alone. One of the worst double grating cases can reduce light extraction efficiency by $9 \%$ with respect to the non-grating case, showing the possibility of an optical field mismatch with the grating structure. To find the absolute maximum, all six of the grating parameters must be swept for both transmission and reflection grating cases simultaneously. 


\section{Chapter 6 - Error Grating GaN LED Simulations}

\subsection{Error Grating GaN LED Model}

We simulate the top and bottom grating model with each cell randomly shifted a distance along the axis in varying degrees of randomization intensity to further understand the effects of fabrication defects on top and bottom gratings. While the widths of holes can be fabricated to great precision, often the placement of holes causes concern, as it affects light extraction efficiency of otherwise ordered photonic crystal structures.

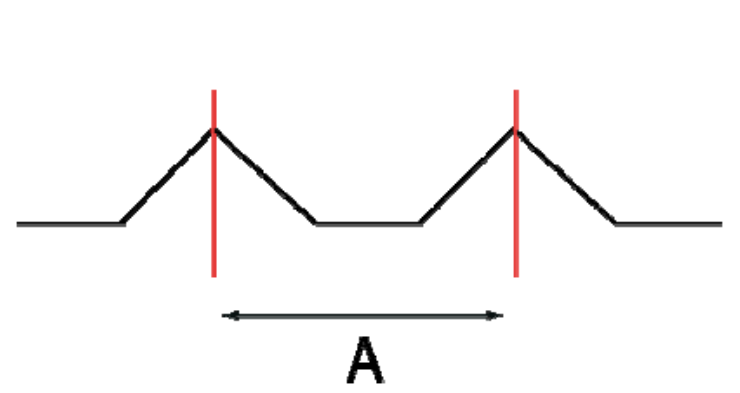

(a)

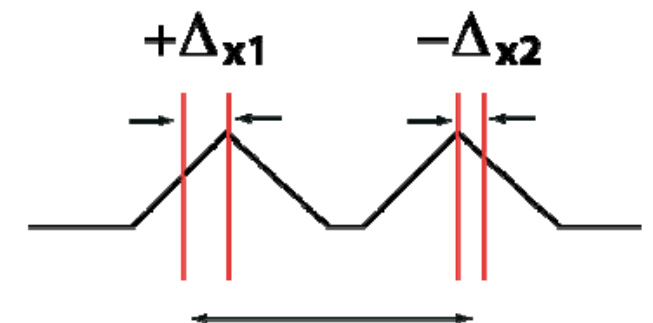

A

(b)

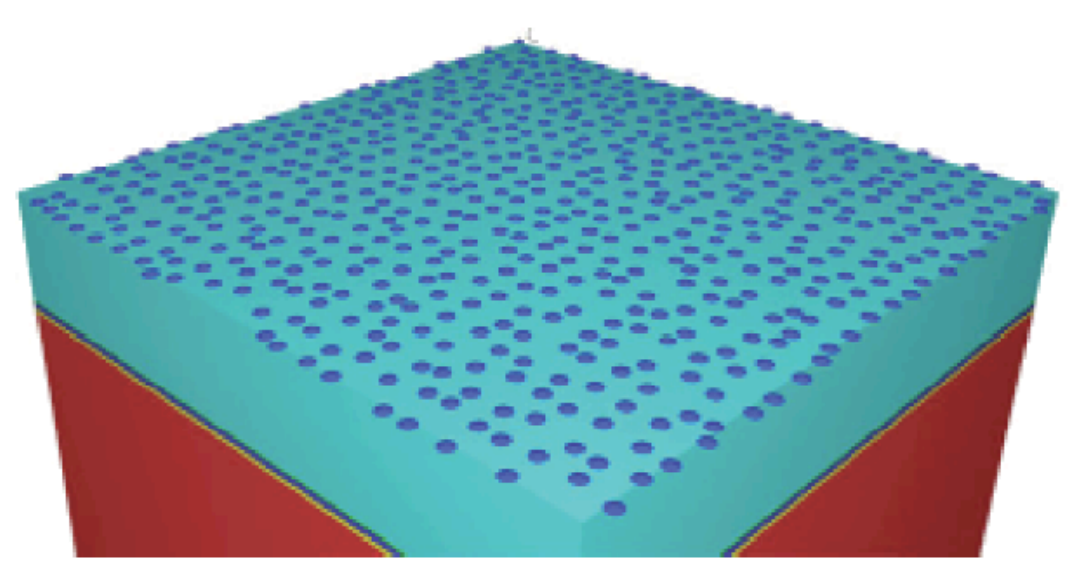

(c)

Fig. 6.1 - Error grating model: (a) normal reference grating model and (b) error grating model with both positive and negative shifts (c) error grating model on GaN LED 
The error grating model still makes use of the 2D FDTD method. Random displacements in position form the basis of the error grating model shown in Fig. 6.1(b) with a normal grating as a reference in Fig. 6.1(a). Displacements can move either direction from the grating cell's original center point. The error grating model shows examples of a positive and negative $\Delta \mathrm{x}$ shift. This randomization then applies to all grating cells in the photonic crystal arrangement with Eq. 8:

$$
\mathrm{x}_{\mathrm{pos}}=\mathrm{N} * \text { period }+(2 * \text { rand }-1) * \mathrm{R} * \text { period, }
$$

where $N$ is an integer index defining the original grating cell location, period is the grating period (A), rand is a pseudo-randomly generated number from 0 to 1 , and $R$ is the randomization factor from 0 to 1 . The quantity $\Delta \mathrm{x}$ in Fig. $6.1(\mathrm{~b})$ represents $(2 *$ rand1)* $R *$ period in Eq. 8. By varying $R$, which applies to all grating cells, from 0 to 1 in 40 steps, the individual rand factor can be emphasized or deemphasized. This process repeats for each of the 12 top and bottom grating models. The top and bottom grating structures place into GaN LED device models as shown in Fig. 6.1(c) (only transmission grating visible) to calculate light extraction efficiency.

\subsection{Error Grating GaN LED Simulation Results}

Randomization in gratings appear to help light extraction efficiency, peaking at about a randomization factor of $R=10 \%$ in most simulations. Fig. 6.2 shows the results for each 
transmission and reflection grating pair. All data normalize according to its $\mathrm{R}=0$ values for easy reading. The graph in Fig. 6.2(a) shows the results for a conical reflection grating matched with each optimal transmission grating. Case No. 12, case No. 8, case No. 11, case No. 7, case No. 10, and case No. 9 in Table 5.2 represent these top-bottom gratings combinations. The graph in Fig. 6.2(b) shows the results for a cylindrical grating paired with each optimal transmission grating in case No. 6, case No. 1, case No. 5, case No. 3, case No.4, and case No. 2. As the randomization intensity factor increases from 0 to 1 , many top and bottom gratings experience an increase in light extraction efficiency around a $10 \%$ displacement of grating cells.

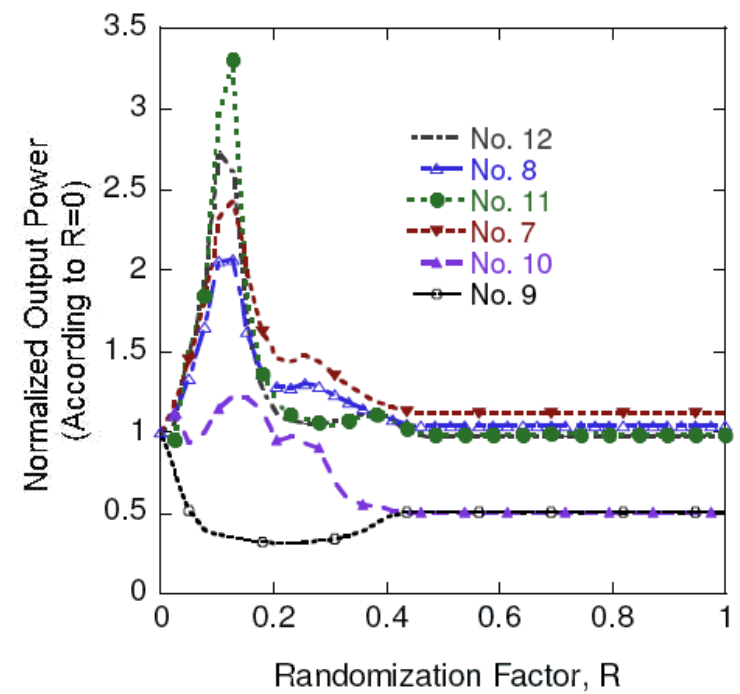

(a)

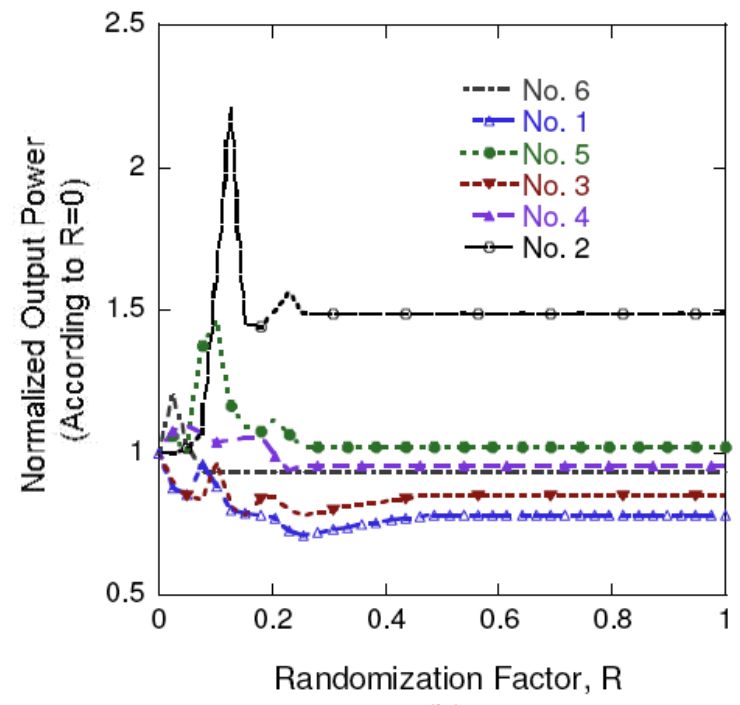

(b)

Fig. 6.2 - Results of error grating simulation for (a) conical reflection gratings paired with transmission gratings: case No. 12, case No. 8, case No. 11, case No. 7, case No. 10, and case No. 9 in Table 5.2. (b) cylindrical grating paired with transmission grating: case No. 6, case No. 1, case No. 5, case No. 3, case No. 4, and case No. 2. in Table 5.2

In essence, a slight random variation or fabrication defect in grating cells would not only be beneficial but desirable for many top and bottom grating types up to a variation 
of $10-15 \%$ for most double grating cases. The non-lossy cylindrical ITO transmission and conical reflection gratings produced the best results at a randomization intensity factor of $12.8 \%$, reaching up to a $230 \%$ improvement over the original top and bottom grating model. Also for most error grating models tested, after $\mathrm{R}=10-15 \%$, light extraction efficiency drops, and after $\mathrm{R}=50 \%$ light extraction efficiency settles to a steady state value.

The randomization creates local variation of the grating structure, A and w. As top and bottom gratings only optimize separately, a small local perturbation could result in more matching gratings and introduce local light extraction improvement. For example, a conical single reflection grating performs worse than a cylindrical grating. Therefore, it has more benefit from randomization of the grating. One case that did not improve at around $\mathrm{R}=10 \%$ resulted from the case of a non-lossy $\mathrm{P}-\mathrm{GaN}$ conical top grating $(\mathrm{A}=1 \mu \mathrm{m}$, $\mathrm{w}=1 \mu \mathrm{m}, \mathrm{d}=138 \mathrm{~nm})$ and the conical reflection grating $(\mathrm{A}=1 \mu \mathrm{m}, \mathrm{w}=1 \mu \mathrm{m}, \mathrm{d}=200 \mathrm{~nm})$. In the single grating simulations, the respective reflection and transmission gratings represent the optimized single grating case, as shown in Table 5.1. Since these gratings were already optimized to the best case in the single grating case, the randomization effect creates destructive interference from the double gratings. It further decreases light extraction efficiency by adding more local destruction or mismatching, hence the drop shown in Fig. 6.2(a), case 9, as the randomization intensity factor increased.

Experimentally, the double grating could give a comparably higher output power as compared with the single grating, when some suitable texturing process applies on both the top ITO/p-GaN and bottom substrate layers. In this thesis, we only prove that the best 
single gratings do not produce the best double grating option when placed in the same LED, as shown in chapter 5 of this thesis. We also show that if fabrication does not attain high periodic uniformity, the light output variation can vary up to $10-15 \%$ and could increase light extraction improvement by $230 \%$ in our double grating error model. Therefore, the experimental data of a double grating can be better than a single grating in either matching grating concepts, error grating effects, or both.

Randomization of grating cells increase light extraction efficiency while having the added benefit of alleviating some of the fabrication complexities demanded by strict periodicities in photonic crystal LEDs. At the time of this thesis, we have not found any papers on the randomization model or our so-called error model in GaN LEDs. We believe the LED error grating model presents a unique model to analyze fabrication defects associated with laser positioning error and randomizations from chemical etching.

\subsection{Error Grating GaN LED Simulation Conclusions}

A fabrication defect of around $10 \%$ actually helps increase light extraction efficiency.

In the best case from our simulation set, an error grating model can achieve a $230 \%$ improvement over the original top and bottom grating model. Not all models exhibit such an increase. In general, fabrication of photonic crystal LEDs do not necessarily need to keep photonic crystal arrangements so ordered and exact. Low intensity random defects in periodic photonic crystals may not only benefit light output, but researchers and manufacturers may also prefer a randomization's desirable effects in many grating structures. 


\section{Chapter 7 - 2-fold Symmetric Transmitted Diffraction Experiments}

We affixed 2-fold symmetric photonic crystal (2PC) structures onto a hemi-cylinder of polydimethylsiloxane (PDMS) and placed the sample on a rotating stage with a green laser beam $(\lambda=532 \mathrm{~nm})$ incident on the grating structure to test these structures by experiment [19]. The $2 \mathrm{PC}$ hole arrays had a lattice pitch of $2 \mu \mathrm{m}$ and were prepared on a silicon wafer with an area of $1 \mathrm{~mm} \times 1 \mathrm{~mm}$, as modeled in Fig. 7.1(b). The 2PC pattern was then transferred onto the planar center point of the PDMS hemi-cylinder by soft nanoimprinting, shown in Fig. 7.1(a). A photodetector was placed behind the grating structure to measure transmission efficiency. Since we interest ourselves only in singlepass transmission characteristics, the hemi-cylinder shape allows reflected and diffracted light to escape with negligible reflection back towards the photodetector.

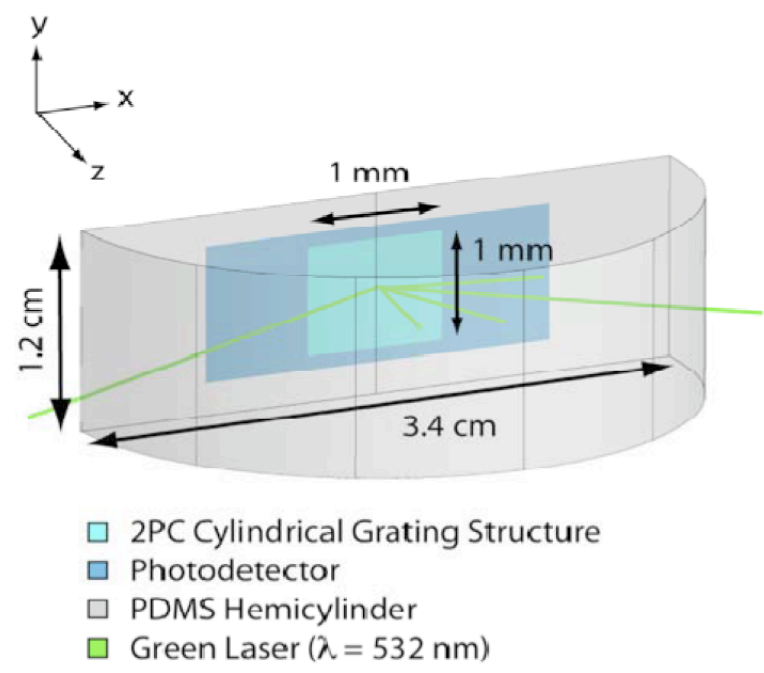

(a)

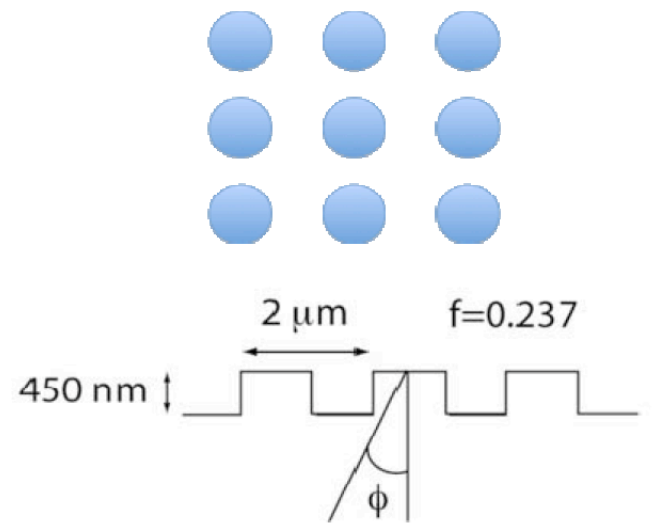

Fig. 7.1 - 2PC Diffraction Experiment Setup: (a) Experimental setup for measuring transmitted diffraction of a $2 P C$ structure mounted on PDMS and (b) 2PC grating structure 


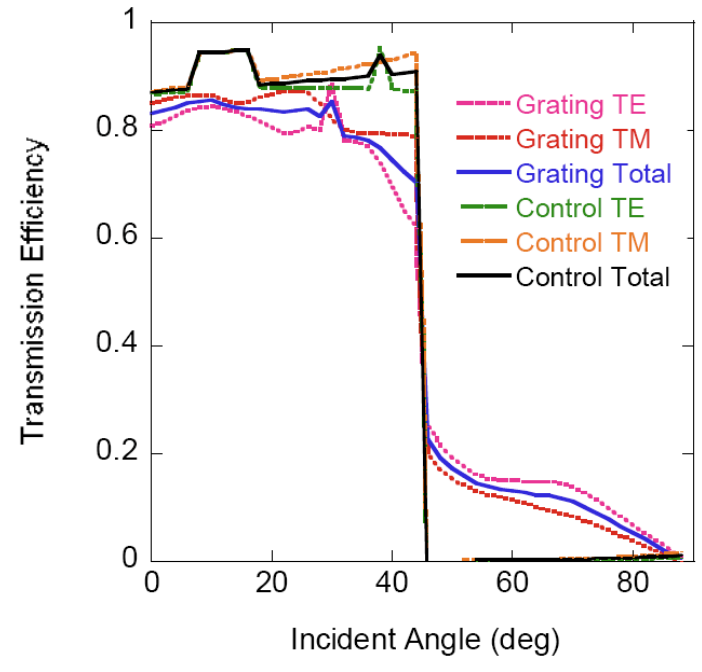

(a)

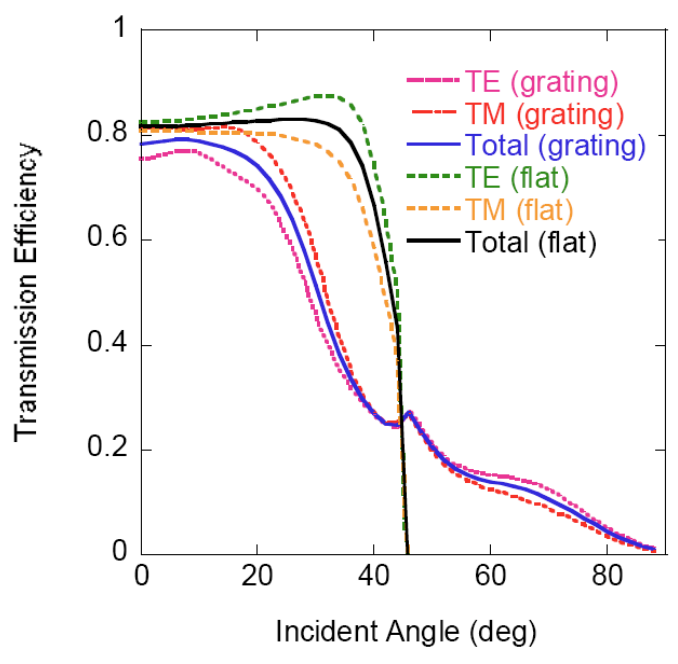

(b)

Fig. 7.2 - FDTD Simulation Results for the 2PC Transmitted Diffraction Experiment: (a) Transmitted Diffraction Efficiency simulated by FDTD simulation and (b) Transmitted Diffraction Efficiency for a non-uniform gridded FDTD simulation

The variable from Fig. 7.1, $f$, represents a "filling factor" as researchers commonly call it in the literature. We have hinted in Chapter 4 that a transmission grating maximizes when $A=w$, or in other words, when we maximize the number of grating cells on the surface of the LED. Therefore, we expect an increase in $f$ will also increase light extraction efficiency.

\subsection{Transmitted Diffraction Experiments and Simulation Results}

Besides the experimental tests, we also develop theoretical models to predict experimental results. Fig. 7.2(a) shows the results of the simulations using the FDTD method. The simulation uses a Yee's mesh and coarsely grids in each dimension at a $\Delta \mathrm{x}$ of $200 \mathrm{~nm}$ and a $\Delta \mathrm{z}$ of $45 \mathrm{~nm}$. A plane wave excites the $2 \mathrm{PC}$ grating and non-grating cases with a Gaussian power distribution. The simulation domain includes 100 grating cells at a 
lattice pitch of $2 \mu \mathrm{m}$, filling factor of 0.237 , and grating cell height of $450 \mathrm{~nm}$. Comparing the simulation results with the experimental ones, we found that FDTD underestimates the experimental data and does not account well for the upward inflection of transmission efficiency about the critical angle. To increase the accuracy, we develop a more finely gridded simulation model around the $2 \mathrm{PC}$ grating. We accomplish this by using nonuniform gridding methods that break apart the simulation domain into finer meshes only at the grating structure, while keeping the rest of the simulation domain coarsely gridded. The non-uniform gridding directly affects the simulation memory and time requirements in the following ways: (1) the non-uniform gridding calculates less points in a simulation domain, and therefore requires less memory, and (2) the time step must be decreased in order to satisfy the Courant stability condition from Eq. 2.4, since the gridding at the 2PC grating shrinks. Remember that decreasing the time step also increases simulation time, but since there constitute fewer points to calculate, the simulation time also decreases. The net change in simulation depends greatly on how many simulation points were eliminated and the time step difference between the uniformly grid and non-uniform grid cases. The simulation only uses a non-uniform grid in the z-direction. We present the non-uniform gridded simulation of the PDMS hemi-cylinder in Fig. 7.2(b). The smoothness of the simulation data for both grating and flat cases results from the fine grid at the grating $(\Delta z=22.5 \mathrm{~nm})$, while the simulation memory requirements were kept low by making the rest of the simulation area coarsely gridded $(\Delta z=56.25 \mathrm{~nm})$. The inflection about an incident angle of 46 degrees agrees very well with the experimental test. 


\subsection{Transmitted Diffraction Experiments and Simulation Conclusions}

In this chapter, we performed transmitted diffraction experiments that showed the effect of diffraction gratings on the transmission across the full angle from 0 to 90 degrees. Experiments agreed well with the theoretical FDTD models, showing a strong critical angle at 46 degrees in the non-grating case, an overall gain of light extraction efficiency integrated over the angular range when using a grating versus the non-grating case. Also, when using a non-uniform gridding technique, the inflection at the critical angle agrees well with the experiment and shows that a diffraction grating allows partial transmission of light, even for angles above the critical angle. 


\section{Chapter 8 - 3-fold Symmetric Photonic Crystals and Flip-Chip Based Designs}

We simulate a higher ordered symmetry photonic crystal grating to further study the effects of photonic crystal structures. The simulations must use a 3-D FDTD method, instead of a 2-D FDTD method, [25,35] for a GaN LED model to calculate final output power. The 2-fold symmetric (ie. square symmetry) conditions enable us to simulate only 2-D simulation domains to obtain the overall effect of the grating, as shown in Fig. 8.1(a). However, we use a 3-fold symmetric photonic crystal grating, which has a geometry shown in Fig. 8.1(b). Therefore, a 3-D FDTD simulation solves the issue of including all optical effects from all neighboring grating cells. We place a time monitor in 5 locations in all simulations: top, front, back, left side, and right side. This allows for a complete picture of the total power output radiating from all facets. The time monitor measured the final average output power from each monitor and summed into a total output power measured in arbitrary units (au) when the total simulation time had elapsed. Note that we omit a bottom monitor, since the Ag reflector plate expects to reflect the vast majority waves back towards one of the other monitors. For thin metal reflectors, only a negligible amount of radiation transmits as evanescent waves. 


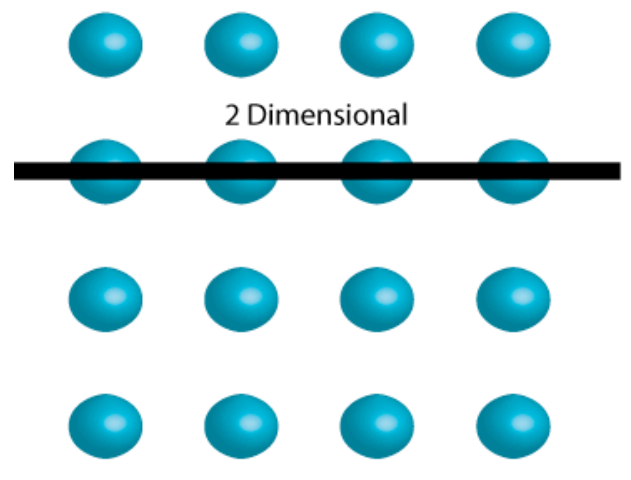

(a)

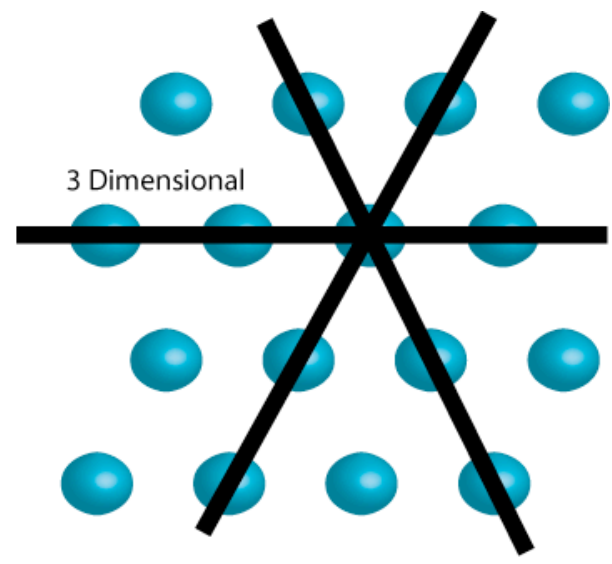

(b)

Fig. 8.1 - Comparison of (a) 2-fold symmetric photonic crystal gratings and (b) 3-fold symmetric photonic crystal gratings

\subsection{3-fold Symmetric Photonic Crystal Grating and GaN LED Models}

Two models constitute this simulation set. The first simulation, a simple semiconductor-air model, aids in discovering the optimized grating parameters. We sweep the parameters with a smaller model since a 3-D FDTD's simulation time and memory requirements expand with simulation domain size as $\mathrm{N}^{4}$ and $\mathrm{N}^{3}$. We can approximate the overall light output by simulating a fraction of the total LED volume. Note that we obtain an improvement percentage based on a ratio of arbitrary units, rather than accurate lumens-per-watt measurements. The massive amount of resources required to simulate 3-D FDTD limits the device size to the amount of RAM and reasonable time we have per simulation. The grating-only simulation lasts for a duration of 500fs, which allows ample time to observe the transmission properties of each grating configuration. We do not need to wait for reflections from a reflector plate in this case. A transmission grating, carved out of the semiconductor surface, can be varied by three variables: grating 
cell-to-cell radius (A), grating cell width (w), and grating height (d). We step through the possible combination physically allowed (ie. $\mathrm{w} \leq \mathrm{A}$ ). For example, the first case would be $\mathrm{A}=1 \mu \mathrm{m}, \mathrm{w}=1 \mu \mathrm{m}, \mathrm{d}=800 \mathrm{~nm}$, then $\mathrm{A}=1 \mu \mathrm{m}, \mathrm{w}=1 \mu \mathrm{m}, \mathrm{d}=1000 \mathrm{~nm}$, and so on. In this model, we take a new approach to sourcing light from the MQW active region. The light generates from random spatial and directional current sources to better represent incoherent light. We place the 32 current sources at random intervals throughout the active layers to provide a pseudorandom distribution. The simulation results in a good representation of a photonic crystal's response to light from all incident angles (ie. 0 to 90 degrees). We setup a new method of arranging light sources, which give gratings large efficiency improvement results compared to other simulation models [25,35], and results in a more comprehensive model for the 3PC simulation.

For the second simulation, a full GaN LED model aids in understanding a diffraction grating's response to a multi-layered thin film LED with an Ag reflector plate. We place the same 3-fold symmetric photonic crystal hole-shaped grating structure made from $\mathrm{p}$ GaN into a full GaN LED model, as shown in Fig. 8.2. In addition, this model represents a flip-chip design that flips the device upside-down and mounts the reflector plate on the $\mathrm{p}-\mathrm{GaN}$ layer rather than the $\mathrm{n}-\mathrm{GaN}$ layer. We apply the same optimal grating parameters from the semiconductor-air simulation in this simulation as the grating parameters for the transmission grating. Only the top 5 cases from the grating optimization simulations take into account for comparison. The simulation time also increases to $2500 \mathrm{fs}$ since we need to account for multiple reflections from the Ag reflector plate in this case. 


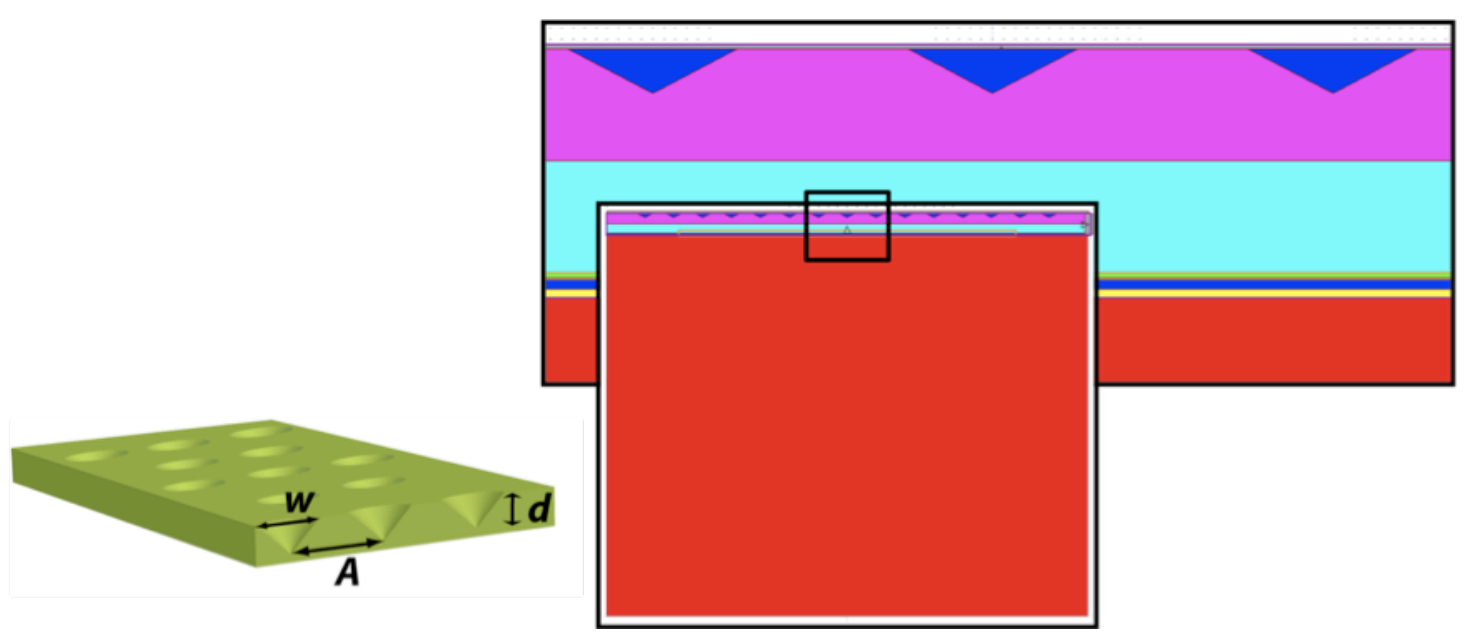

Fig. 8.2 - 3-Fold Symmetric Photonic Crystal GaN LED Model

\subsection{3-fold Symmetric Photonic Crystal GaN LED Simulation Results}

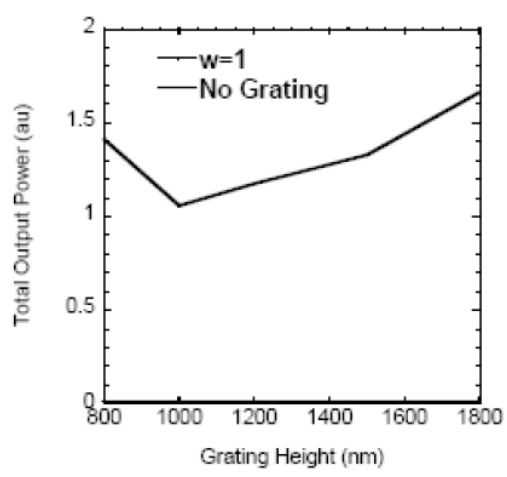

(a)

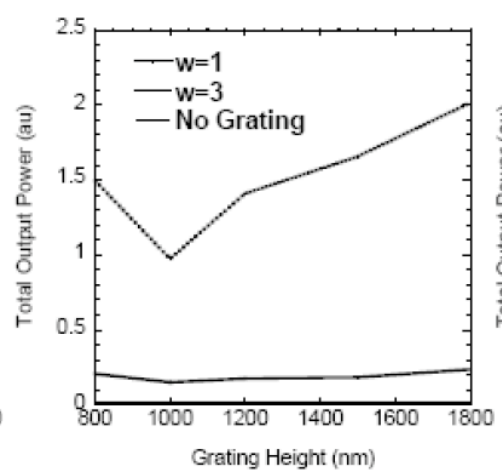

(b)

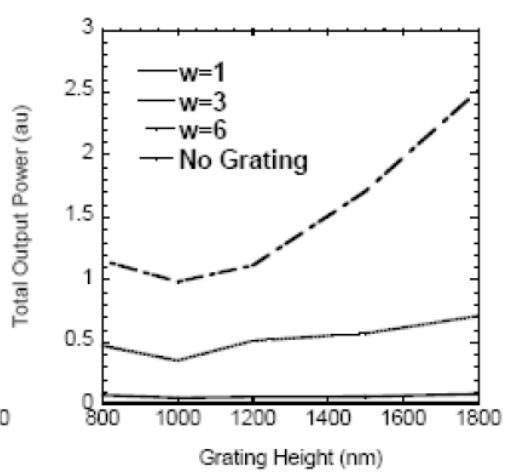

(c)

Fig. 8.3 - Total output power for a GaN-to-Air grating structure: (a) A=1 $\mu$ m., (b) $A=3 \mu m$., and (c) $A=6 \mu m$

From Chapter 4, we discover that a transmission grating optimizes when $A=w$, in other words, when unit cells acquire the most area on the LED surface. In recent literature, researchers call this a filling factor and can be described as the ratio of the total area covered by the unit cells and the total top surface area. 
Now, we turn our attention to the semiconductor-air simulations. Fig. 8.3 shows the results of the 3-D FDTD method simulations, limited to the interface between $\mathrm{GaN}$ and air. A grating with the parameters $A, w$, and $d$, varies in steps. As the grating height increases, the total output power also increases. The improvement, in all cases of $A=1,3$, and $6 \mu \mathrm{m}$, at $d=800 \mathrm{~nm}$ indicates that the relationship between $d$ and output power may have a sinusoidal-like relationship.

Table 8.1 - Full GaN Model 3-D FDTD Simulations for Best Case Gratings

\begin{tabular}{|c|c|c|c|c|c|}
\hline $\mathbf{A}(\mathbf{u m})$ & $\mathbf{w}(\mathbf{u m})$ & $\mathbf{d}(\mathbf{n m})$ & $\begin{array}{l}\mathbf{P}\left(|\mathbf{E}|^{2}, \mathbf{a u}\right) \text { of the } \\
\text { Grating-Only }\end{array}$ & $\begin{array}{l}\mathbf{P}\left(|\mathbf{E}|^{2}, \text { au) of the Full }\right. \\
\text { GaN Model }\end{array}$ & $\begin{array}{l}\text { Improvement factor for Full } \\
\text { GaN Model }\end{array}$ \\
\hline 3 & 3 & 1800 & 2.010 & 43.292 & 43.77 \\
1 & 1 & 1800 & 1.658 & 42.381 & 42.83 \\
3 & 3 & 1500 & 1.651 & 41.165 & 41.57 \\
6 & 6 & 1500 & 1.703 & 40.807 & 41.20 \\
6 & 6 & 1800 & 2.508 & 40.359 & 40.74 \\
\hline \multicolumn{7}{|r|}{} \\
No Grating
\end{tabular}

Next, a 3-D FDTD full GaN LED model was simulated with the best cases of the grating-only simulations. Table 8.1 shows these best cases and their results for the full GaN model. The grating with the characteristics of $A=3 \mu \mathrm{m}, w=3 \mu \mathrm{m}, d=1800 \mathrm{~nm}$ produces the best transmission grating. Even though the $A=6 \mu \mathrm{m}, w=6 \mu \mathrm{m}, d=1800 \mathrm{~nm}$ grating maximized light extraction improvement in the grating-only simulations, the results in the full GaN LED model indicate that it performed the worst out of all 5 gratings simulated. Therefore, grating-only simulations provide insufficiently optimized grating structures when considered within full GaN LED models. Internal modes must be considered, as well as multiple reflections. The grating provides a method of escape for incoherent light generated within the device. Since the FDTD method steps in time, it can visually draw an accurate picture of what a simulation domain looks like at the end of a simulation. This presents a myriad of uses for discovering field effects of a grating 
structure, such as freeze frames of $\mathrm{E}$ and $\mathrm{H}$ fields in the entire spatial domain, animations of the time propagation of fields, and mode profiles. For example, Fig. 8.4(a) and (b), which show the xy-slice at the time monitor and the xz-slice at the center of the LED, also reveal light escaping from the holes that compose the transmission grating! Evidently, grating structures must be closely packed together to maximize light extraction efficiency, since the escaping fields from the top only emit strongly from the grating cells themselves.

This simulation also reveals another interesting and practical method of calculating far-field patterns. Since the grating structure itself approximates the pattern of light output in the near field, it is feasible that our understanding of the geometry of the grating alone could provide enough information to obtain the far-field pattern of the LED. We solve for the far-field intensity by using scalar diffraction theory. We also place some restrictions and assumptions on the model: (1) that the emission angle does not vary too widely and (2) that Eq. 9 holds true:

$$
z>\frac{2 D^{2}}{\lambda}=\frac{2(100 \mu \mathrm{m})^{2}}{460 \mathrm{~nm}}=4.348 \mathrm{~cm},
$$

where $D$ is the longest linear dimension of the grating structure and $\lambda$ is the wavelength of light $(460 \mathrm{~nm})$. This distance comes from the so-called "antenna designer's formula and defines the region in which the Fraunhofer approximation can be applied. The z-axis points in a direction directly normal to the surface of the LED in this simulation. If we assume all of these conditions, then the intensity of the far-field optical field distribution simply results from the Fourier transform of the geometry of the 
diffraction grating itself [40].

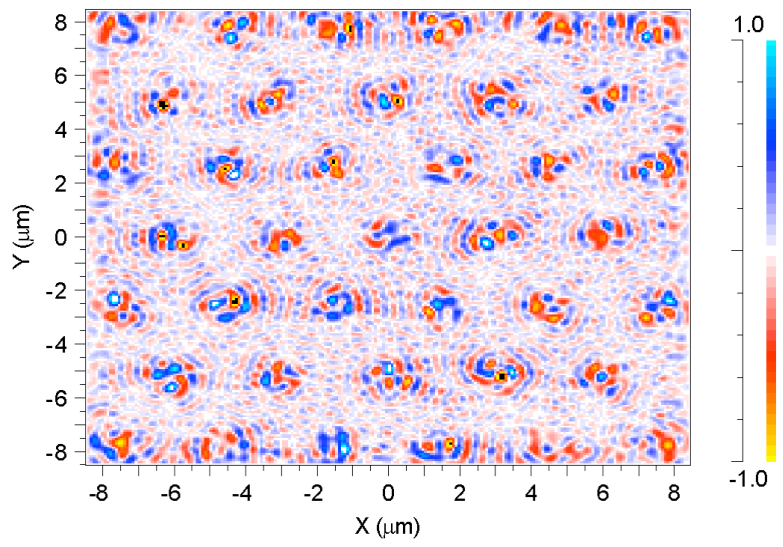

(a)

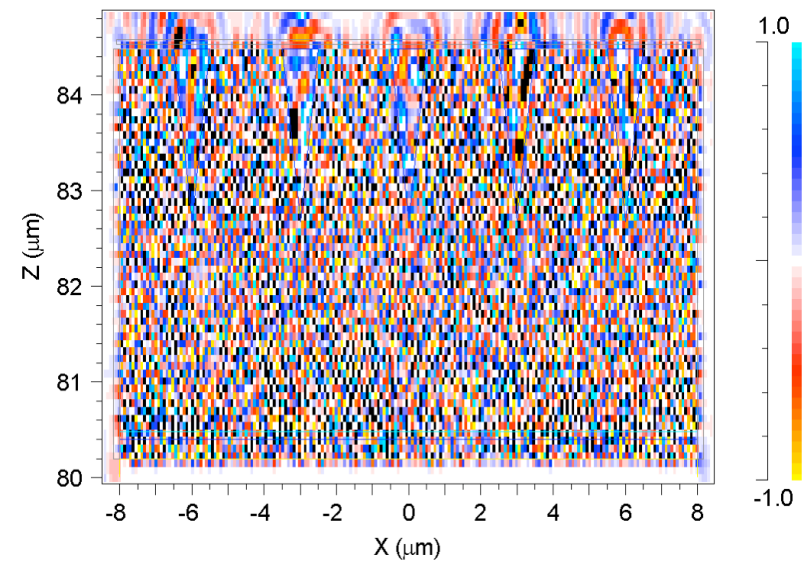

(b)

Fig. 8.4 - Simulation Domain from a 3-fold Symmetric Photonic Crystal Grating Simulation: (a) xyplane cut, (b) xz-plane cut 


\subsection{3-Fold Symmetric Photonic Crystal Results Summary and Conclusions}

In this chapter, we present a 3-D FDTD simulation of a GaN-air grating interface and a full LED model with the gratings on top. The gratings for a 3-D FDTD model again indicate the same result of the 2-D FDTD transmission grating models: as $A$ approaches the dimensions of $w$, light extraction efficiency improved. With 3-D simulations and a more accurate source combination that simulated incoherent lighting, we could clearly see the effects of optimized gratings, especially when observing the simulation domain at the end of the simulation. The best case grating in the full LED simulation set in Table 8.2 was $A=3 \mu \mathrm{m}, \mathrm{w}=3 \mu \mathrm{m}, \mathrm{d}=1800 \mathrm{~nm}$. Light was extracted from the holes in the structure and emitted out of the top. This output was captured at the top of the LED in the near field region. Since the light escaping from the LED approximately matched the geometry of the grating itself, a far-field pattern could be obtained by simply knowing the geometrical structure of the transmission grating itself and also applying the Fraunhofer approximation to obtain far-field diffraction and intensity patterns. 


\section{Chapter 9 - Overall Conclusions and Future Work}

\subsection{Overall Conclusions for Each Grating Type}

In chapter 3, a simulation model used the FDTD method based on a Yee mesh to calculate GaN LED light extraction efficiency. The simulation results showed that the cylindrical grating case had a $111 \%$ improvement of the light extraction, and the conical grating case had a $112 \%$ improvement compared to the flat plate case. The highest efficiency in a reflection grating resulted when $w=1 \mu \mathrm{m}$. As $w$ became small compared to $A$, the maximum average output power increased. A designer of reflection gratings should consider making the grating with a larger grating period, (i.e. $A=6 \mu \mathrm{m}$ ), smaller grating width, (i.e. $w=1 \mu \mathrm{m}$ ), and a grating height $d$ at around $158 \mathrm{~nm}$ for both conical- or cylindrical-hole reflection gratings.

In chapter 4, single transmission gratings were simulated using the 2-D FDTD method. Three material types were simulated: non-lossy p-GaN, non-lossy ITO, and lossy ITO. In addition, a conical and cylindrical grating cell shape was simulated for each type of material. This research has found what has already been agreed to in the literature, that grating structures influence light extraction efficiency in the following way: as $A$ approached the dimensions of $w$, light extraction efficiency improved. In other words, when grating cells were closely packed, maximum light extraction efficiency occurred.

In chapter 5, we simulated top and bottom grating structures, taking optimized single gratings from the optimized cases of transmission and reflection gratings alone. In general, grating structures improved light extraction of LEDs compared to the nongrating structure. The simulation results also showed that the optimized single grating 
optical fields were altered significantly by the introduction of a second grating structure. The case 1, Table 5.2 double grating, with sequential, single variable optimizations, could achieve a light extraction improvement of $118 \%$ with respect to the non-grating case that outperformed either single grating alone. Similar optimizations could have been done for the other cases.

In chapter 6 , we simulated an error grating model to study the positional defect in ordered photonic crystal gratings. A randomization factor of around $10 \%$ helped increase light extraction efficiency. In the best case, light extraction efficiency improved $230 \%$ over the original top and bottom grating model. Low intensity random defects in periodic photonic crystals not only benefited light extraction but produced desirable effects in many grating structures.

In chapter 7, we performed transmitted diffraction experiments that showed the effect of diffraction gratings on the transmission across the full angle from 0 to 90 degrees. Experiments agreed well with the theoretical FDTD models, showing a strong critical angle at 46 degrees and an overall gain of light extraction efficiency integrated over the angular range when using a grating versus the non-grating case. Also, a non-uniform grid revealed the inflection about the critical angle in the grating case. This allowed for trapped light to escape when contacting a grating at angles above the critical angle.

Finally, in chapter 8, a 3-D FDTD simulation was presented of a GaN-air grating interface and a full LED model with the gratings on top. The gratings for a 3-D FDTD model again indicated the same result of the 2-D FDTD transmission grating models: as $A$ approached the dimensions of $w$, light extraction efficiency improved. With 3-D 
simulations and a more accurate source combination that simulated incoherent lighting, we could clearly see the effects of optimized gratings, especially when observing the simulation domain at the end of the simulation. The best case grating in the full LED simulation set optimized light extraction efficiency at $A=3 \mu \mathrm{m}, \mathrm{w}=3 \mu \mathrm{m}, \mathrm{d}=1800 \mathrm{~nm}$. Rendered drawings of the simulation domain revealed that light was extracted from the holes in the structure and emitted out of the top. This output was captured at the top of the LED in the near field region. Since the position of the light escaping from the LED closely followed the geometry of the grating itself, a far-field pattern could be obtained by simply knowing the geometrical structure of the transmission grating itself and using the Fraunhofer approximation for far-field diffraction and intensity patterns.

\subsection{Future Work}

\subsubsection{2-fold Symmetric Photonic Quasicrystals}

First, in addition to 3-fold symmetric photonic crystals, many other crystal types exist that could be researched. Particularly, the area of photonic quasicrystal (PQC) research provides the most promising geometries for light extraction from LEDs [36]. Photonic quasicrystals differ from photonic crystals in that the geometry cannot be translationally symmetric. Rather, photonic quasicrystals exhibit period symmetry by rotational symmetry and can therefore achieve higher orders of symmetry than standard crystal structures. Fig. 9.1 below shows an example of a few 12-fold symmetric photonic quasicrystals, where colored polygons have been added to better show the structure and symmetry of the photonic quasicrystal. Fig. 9.1(a) is a hexagonal packed structure, while 
Fig. 9.1(b) is a circular packed structure. According to reference [36], 12PQC grating structures outperform 2PC grating structures in light output experiments. Varying symmetries affect the far-field pattern, total light extraction efficiency, and subwavelength feature sizes, but researchers must simulate more LED structures to discover those effects.

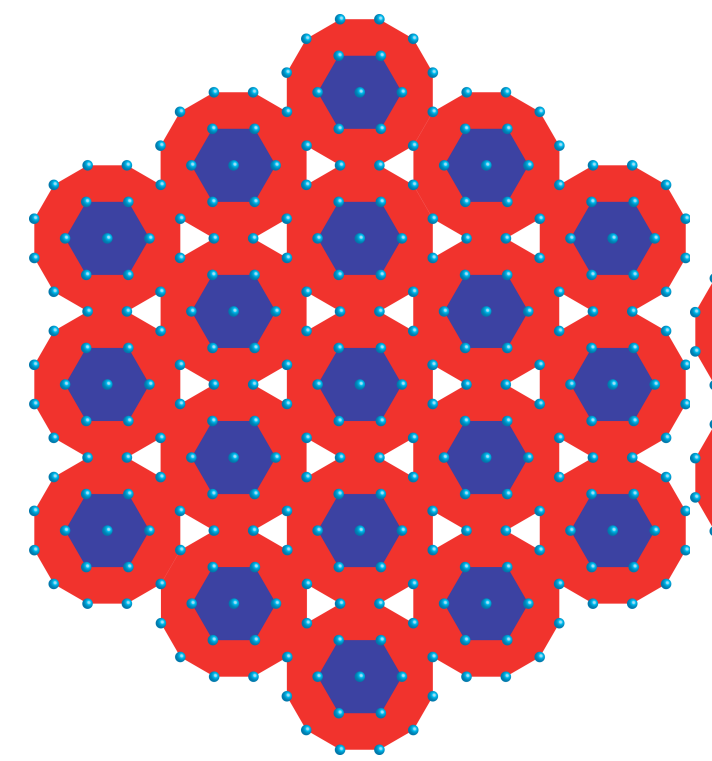

(a)

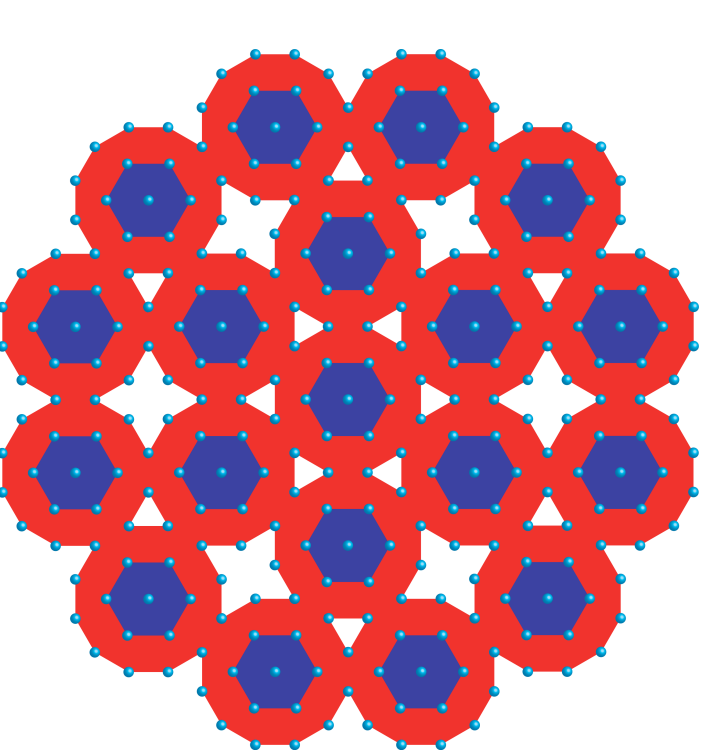

(b)

Fig. 9.1 - 12-fold Symmetric Photonic Quasicrystals: (a) hexagonal packed geometry, (b) circular packed geometry

\subsubsection{Nano-scale Grating Structures}

In addition to differing symmetries, the topic of nano-scale grating structures exploration. How do sub-wavelength optics affect light extraction efficiency? In recent literature, the concept of a photonic band gap (PBG) occurs at sub-wavelength optics when photonic crystals block certain frequencies of light from passing through the structure. Photons at specific frequencies are denied passage in much the same way that 
certain energy levels within a semiconductor forbid electrons from occupying those energy levels $[37,38]$. This happens because of the periodic, internal regions of varying dielectric constants in the atomic structure. Alternating dielectric regions allow only certain light frequencies to pass through. These could be used to make reflection grating structures and/or transmission grating structures for LEDs to allow maximal light to escape from the LED. We fully expect very interesting results and novel research to come from simulations from photonic quasicrystal arrangements. More importantly, optimization trends and guidelines can be formed to guide fabricators to produce better quality high brightness LEDs (HB-LED). These guidelines would be very novel, and currently, no research exists that has produced such results.

\subsubsection{Nano-dot Bump Defects}

When a hole/pillar forms, the geometry forms imperfect conical or cylindrical shapes. While we have shown that positional defects in our error grating models can be beneficial for light extraction efficiency, we must discover what nano-dot bump defects would do to light extraction efficiency on an LED. A bump defect would be similar to a nanoroughening of the surface, small variations in height that naturally occur during the fabrication process. These improvements may help fabricators to better understand the importance of surface uniformity and its effects on light extraction.

\subsubsection{Genetic Mutation Algorithms to Determine Absolute Maxima}

Finally, top and bottom grating designs constitute an important research step towards 
higher luminosity and higher efficiency LEDs. Top and bottom grating LEDs present significant difficulties when sweeping grating parameters in an LED model. The "brute force" method that scans all parameters results in an overly cumbersome process that scales very poorly with more and more gratings. Genetic mutation algorithms in the field of computational intelligence can aid in finding the absolute maximums in systems with many variables. Mutation allows the algorithm to avoid local minima by preventing the "population" of grating combinations from becoming too similar, thereby slowing or even stopping evolution towards higher maximums of light extraction efficiency. If such an algorithm could be programmed and scripted with RSoft's MOST optimizer, then this would greatly reduce the simulation attempts to find maxima and also provide a feasible method to actually obtain the absolute maximum.

The area of photonic research rapidly expands, especially with consumers demanding higher bitrates and faster switching. In fact, recent development in optical transistors and optical computers may very well outdate the electron as the most popular medium of information transfer. Therefore, LEDs and LDs will play a significant role in the future of information transfer. Diodes with greater efficiency, luminosity, and heat-sinking capability must be researched vigorously. 
APPENDIX A: SUMMARY OF GRATING SIMULATIONS BY RANK

\begin{tabular}{|c|c|c|c|c|c|c|c|c|c|c|}
\hline Rank & $\begin{array}{l}\text { Structure Name } \\
\text { Top Transmission }\end{array}$ & $\begin{array}{l}\mathbf{A} \\
\mu m\end{array}$ & $\begin{array}{l}\mathbf{w} \\
\mu m\end{array}$ & $\begin{array}{l}\text { d } \\
n m\end{array}$ & $\begin{array}{l}\text { Structure Name } \\
\text { Bottom Reflection }\end{array}$ & $\mu m$ & $\begin{array}{l}\mathbf{w} \\
\mu m\end{array}$ & $\begin{array}{l}\mathbf{d} \\
n m\end{array}$ & $\begin{array}{l}\text { Power } \\
\text { (au) }\end{array}$ & $\begin{array}{l}\text { Light Ext. } \\
\text { Eff. Imp. } \\
* *\end{array}$ \\
\hline 1 & $\begin{array}{c}\text { Non-Lossy } \\
\text { Conical P-GaN } \\
\text { Non-Lossy }\end{array}$ & 1 & 1 & 138 & None & - & - & - & 12.374 & $165 \%$ \\
\hline 2 & $\begin{array}{c}\text { Cylindrical P-GaN } \\
\text { Non-Lossy }\end{array}$ & 2 & 1 & $151^{*}$ & None & - & - & - & 11.589 & $149 \%$ \\
\hline 3 & $\begin{array}{c}\text { Cylindrical P-GaN } \\
\text { Non-Lossy }\end{array}$ & 4 & 3 & 156 & None & - & - & - & 10.926 & $134 \%$ \\
\hline 4 & $\begin{array}{c}\text { Cylindrical P-GaN } \\
\text { Non-Lossy }\end{array}$ & 3 & 2 & 156 & None & - & - & - & 10.897 & $134 \%$ \\
\hline 5 & $\begin{array}{l}\text { Cylindrical P-GaN } \\
\text { Non-Lossy }\end{array}$ & 5 & 4 & 156 & None & - & - & - & 10.722 & $130 \%$ \\
\hline 6 & $\begin{array}{l}\text { Cylindrical P-GaN } \\
\text { Non-Lossy }\end{array}$ & 6 & 5 & 156 & None & - & - & - & 10.473 & $125 \%$ \\
\hline 7 & $\begin{array}{c}\text { Cylindrical P-GaN } \\
\text { Non-Lossy }\end{array}$ & 6 & 4 & 156 & None & - & - & - & 10.341 & $122 \%$ \\
\hline 8 & $\begin{array}{c}\text { Cylindrical P-GaN } \\
\text { Non-Lossy }\end{array}$ & 6 & 3 & 164 & None & - & - & - & 10.3 & $121 \%$ \\
\hline 9 & $\begin{array}{l}\text { Cylindrical P-GaN } \\
\text { Lossy Conical }\end{array}$ & 5 & 3 & 156 & None & - & - & - & 10.225 & $119 \%$ \\
\hline 10 & $\begin{array}{c}\text { ITO }^{1} \\
\text { Non-Lossy }\end{array}$ & 4 & 4 & 52 & Cylindrical & 4 & 1 & 128 & 10.14 & $118 \%$ \\
\hline 11 & $\begin{array}{c}\text { Cylindrical P-GaN } \\
\text { Non-Lossy }\end{array}$ & 3 & 1 & 156 & None & - & - & - & 10.025 & $115 \%$ \\
\hline 12 & $\begin{array}{l}\text { Cylindrical P-GaN } \\
\text { Non-Lossy }\end{array}$ & 5 & 1 & 184 & None & - & - & - & 9.9903 & $114 \%$ \\
\hline 13 & $\begin{array}{c}\text { Cylindrical P-GaN } \\
\text { Non-Lossy }\end{array}$ & 4 & 1 & 180 & None & - & - & - & 9.9703 & $114 \%$ \\
\hline 14 & $\begin{array}{c}\text { Cylindrical P-GaN } \\
\text { Non-Lossy }\end{array}$ & 1 & 1 & 156 & None & - & - & - & 9.9516 & $114 \%$ \\
\hline 15 & $\begin{array}{l}\text { Cylindrical P-GaN } \\
\text { Non-Lossy }\end{array}$ & 2 & 2 & 156 & None & - & - & - & 9.9516 & $114 \%$ \\
\hline 16 & $\begin{array}{l}\text { Cylindrical P-GaN } \\
\text { Non-Lossy }\end{array}$ & 3 & 3 & 164 & None & - & - & - & 9.9516 & $114 \%$ \\
\hline 17 & $\begin{array}{l}\text { Cylindrical P-GaN } \\
\text { Non-Lossy }\end{array}$ & 4 & 4 & 156 & None & - & - & - & 9.9516 & $114 \%$ \\
\hline 18 & $\begin{array}{c}\text { Cylindrical P-GaN } \\
\text { Non-Lossy }\end{array}$ & 5 & 5 & 156 & None & - & - & - & 9.9516 & $114 \%$ \\
\hline 19 & Cylindrical P-GaN & 6 & 6 & 156 & None & - & - & - & 9.9516 & $114 \%$ \\
\hline 20 & $\begin{array}{c}\text { None } \\
\text { Non-Lossy }\end{array}$ & - & - & - & Conical & 1 & 1 & 200 & 10.13 & $112 \%$ \\
\hline 21 & $\begin{array}{l}\text { Cylindrical P-GaN } \\
\text { Non-Lossy }\end{array}$ & 5 & 2 & 180 & None & - & - & - & 9.8757 & $112 \%$ \\
\hline 22 & $\begin{array}{c}\text { Conical P-GaN } \\
\text { Non-Lossy }\end{array}$ & 2 & 1 & 132 & None & - & - & - & 9.846 & $111 \%$ \\
\hline 23 & $\begin{array}{c}\text { Cylindrical P-GaN } \\
\text { Non-Lossy }\end{array}$ & 4 & 2 & 156 & None & - & - & - & 9.8391 & $111 \%$ \\
\hline 24 & Cylindrical P-GaN & 6 & 2 & 164 & None & - & - & - & 9.8157 & $111 \%$ \\
\hline 25 & $\begin{array}{c}\text { None } \\
\text { Non-Lossy }\end{array}$ & - & - & - & Cylindrical & 2 & 1 & 136 & 9.8135 & $111 \%$ \\
\hline 26 & $\begin{array}{c}\text { Cylindrical ITO } \\
\text { Non-Lossy }\end{array}$ & 4 & 4 & 126 & None & - & - & - & 9.7258 & $109 \%$ \\
\hline 27 & Cylindrical ITO & 1 & 1 & 126 & None & - & - & - & 9.7258 & $109 \%$ \\
\hline
\end{tabular}




\begin{tabular}{|c|c|c|c|c|c|c|c|c|c|c|}
\hline Rank & $\begin{array}{l}\text { Structure Name } \\
\text { Top Transmission }\end{array}$ & $\begin{array}{l}\mathbf{A} \\
\mu m\end{array}$ & $\begin{array}{l}\mathbf{w} \\
\mu m\end{array}$ & $\begin{array}{l}\mathbf{d} \\
n m\end{array}$ & $\begin{array}{l}\text { Structure Name } \\
\text { Bottom Reflection }\end{array}$ & $\begin{array}{l}\mathbf{A} \\
\mu m\end{array}$ & $\begin{array}{l}\mathbf{w} \\
\mu m\end{array}$ & $\begin{array}{l}\mathbf{d} \\
n m\end{array}$ & $\begin{array}{l}\text { Power } \\
\text { (au) }\end{array}$ & $\begin{array}{l}\text { Light Ext. } \\
\text { Eff. Imp. } \\
* *\end{array}$ \\
\hline 28 & $\begin{array}{c}\text { Non-Lossy } \\
\text { Cylindrical ITO } \\
\text { Non-Lossy }\end{array}$ & 2 & 2 & 126 & None & - & - & - & 9.7258 & $109 \%$ \\
\hline 29 & $\begin{array}{c}\text { Cylindrical ITO } \\
\text { Non-Lossy }\end{array}$ & 3 & 3 & 126 & None & - & - & - & 9.7258 & $109 \%$ \\
\hline 30 & $\begin{array}{c}\text { Cylindrical ITO } \\
\text { Non-Lossy }\end{array}$ & 5 & 5 & 126 & None & - & - & - & 9.7258 & $109 \%$ \\
\hline 31 & Cylindrical ITO & 6 & 6 & 126 & None & - & - & - & 9.7258 & $109 \%$ \\
\hline 32 & None & - & - & - & Cylindrical & 5 & 1 & 174 & 9.4703 & $103 \%$ \\
\hline 33 & $\begin{array}{c}\text { None } \\
\text { Non-Lossy }\end{array}$ & - & - & - & Cylindrical & 6 & 1 & 174 & 9.448 & $103 \%$ \\
\hline 34 & Cylindrical ITO & 6 & 5 & 132 & None & - & - & - & 9.4149 & $102 \%$ \\
\hline 35 & None & - & - & - & Conical & 6 & 1 & 164 & 9.3048 & $100 \%$ \\
\hline 36 & None & - & - & - & Cylindrical & 4 & 1 & 174 & 9.2998 & $100 \%$ \\
\hline 37 & None & - & - & - & Conical & 5 & 1 & 158 & 9.2972 & $99 \%$ \\
\hline 38 & None & - & - & - & Cylindrical & 5 & 2 & 174 & 9.2967 & $99 \%$ \\
\hline 39 & $\begin{array}{c}\text { None } \\
\text { Non-Lossy }\end{array}$ & - & - & - & Cylindrical & 3 & 1 & 174 & 9.2788 & $99 \%$ \\
\hline 40 & Cylindrical ITO & 5 & 4 & 132 & None & - & - & - & 9.2711 & $99 \%$ \\
\hline 41 & $\begin{array}{c}\text { None } \\
\text { Non-Lossy }\end{array}$ & - & - & - & Cylindrical & 6 & 2 & 174 & 9.248 & $98 \%$ \\
\hline 42 & Cylindrical ITO & 4 & 3 & 132 & None & - & - & - & 9.1355 & $96 \%$ \\
\hline 43 & $\begin{array}{c}\text { None } \\
\text { Non-Lossy }\end{array}$ & - & - & - & Conical & 6 & 2 & 164 & 9.123 & $96 \%$ \\
\hline 44 & Conical P-GaN & 3 & 1 & 156 & None & - & - & - & 9.0889 & $95 \%$ \\
\hline 45 & None & - & - & - & Cylindrical & 6 & 3 & 174 & 9.0882 & $95 \%$ \\
\hline 46 & $\begin{array}{c}\text { None } \\
\text { Lossy Cylindrical }\end{array}$ & - & - & - & Conical & 5 & 2 & 164 & 9.0849 & $95 \%$ \\
\hline 47 & $\begin{array}{l}\text { ITO } \\
\text { Lossy Cylindrical }\end{array}$ & 1 & 1 & 118 & None & - & - & - & 9.0752 & $95 \%$ \\
\hline 48 & $\begin{array}{ll} & \text { ITO } \\
\text { Lossy Cylindrical }\end{array}$ & 2 & 2 & 118 & None & - & - & - & 9.0752 & $95 \%$ \\
\hline 49 & $\begin{array}{l}\text { ITO } \\
\text { Lossy Cylindrical }\end{array}$ & 3 & 3 & 118 & None & - & - & - & 9.0752 & $95 \%$ \\
\hline 50 & $\begin{array}{l}\text { ITO } \\
\text { Lossy Cylindrical }\end{array}$ & 4 & 4 & 118 & None & - & - & - & 9.0752 & $95 \%$ \\
\hline 51 & $\begin{array}{l}\text { ITO } \\
\text { Lossy Cylindrical }\end{array}$ & 5 & 5 & 118 & None & - & - & - & 9.0752 & $95 \%$ \\
\hline 52 & $\begin{array}{c}\text { ITO } \\
\text { Non-Lossy }\end{array}$ & 6 & 6 & 118 & None & - & - & - & 9.0752 & $95 \%$ \\
\hline 53 & $\begin{array}{l}\text { Cylindrical ITO } \\
\text { Non-Lossy }\end{array}$ & 6 & 4 & 132 & None & - & - & - & 9.0485 & $94 \%$ \\
\hline 54 & Cylindrical P-GaN & 6 & 1 & 152 & None & - & - & - & 9.0433 & $94 \%$ \\
\hline 55 & None & - & - & - & Cylindrical & 3 & 2 & 132 & 9.023 & $94 \%$ \\
\hline 56 & None & - & - & - & Cylindrical & 5 & 3 & 174 & 9.0073 & $93 \%$ \\
\hline 57 & None & - & - & - & Cylindrical & 4 & 3 & 182 & 8.9952 & $93 \%$ \\
\hline 58 & $\begin{array}{l}\text { None } \\
\text { Non-Lossy }\end{array}$ & - & - & - & Cylindrical & 5 & 4 & 182 & 8.9929 & $93 \%$ \\
\hline 59 & Conical P-GaN & 5 & 1 & 200 & None & - & - & - & 8.9828 & $93 \%$ \\
\hline 60 & $\begin{array}{l}\text { None } \\
\text { Non-Lossy }\end{array}$ & - & - & - & Cylindrical & 4 & 2 & 174 & 8.982 & $93 \%$ \\
\hline 61 & Conical P-GaN & 4 & 1 & 176 & None & - & - & - & 8.962 & $92 \%$ \\
\hline 62 & None & - & - & - & Cylindrical & 1 & 1 & 182 & 8.9414 & $92 \%$ \\
\hline
\end{tabular}




\begin{tabular}{|c|c|c|c|c|c|c|c|c|c|c|}
\hline Rank & $\begin{array}{l}\text { Structure Name } \\
\text { Top Transmission }\end{array}$ & $\begin{array}{l}\mathbf{A} \\
\mu m\end{array}$ & $\begin{array}{l}\mathbf{w} \\
\mu m\end{array}$ & $\begin{array}{l}\mathbf{d} \\
n m\end{array}$ & $\begin{array}{l}\text { Structure Name } \\
\text { Bottom Reflection }\end{array}$ & $\begin{array}{l}\mathbf{A} \\
\mu m\end{array}$ & $\begin{array}{l}\mathbf{w} \\
\mu m\end{array}$ & $\begin{array}{l}\mathbf{d} \\
n m\end{array}$ & $\begin{array}{l}\text { Power } \\
\text { (au) }\end{array}$ & $\begin{array}{l}\text { Light Ext. } \\
\text { Eff. Imp. } \\
* *\end{array}$ \\
\hline 63 & None & - & - & - & Cylindrical & 2 & 2 & 182 & 8.9414 & $92 \%$ \\
\hline 64 & None & - & - & - & Cylindrical & 3 & 3 & 182 & 8.9414 & $92 \%$ \\
\hline 65 & None & - & - & - & Cylindrical & 4 & 4 & 182 & 8.9414 & $92 \%$ \\
\hline 66 & None & - & - & - & Cylindrical & 5 & 5 & 182 & 8.9414 & $92 \%$ \\
\hline 67 & None & - & - & - & Cylindrical & 6 & 6 & 182 & 8.9414 & $92 \%$ \\
\hline 68 & None & - & - & - & Cylindrical & 6 & 4 & 182 & 8.9368 & $92 \%$ \\
\hline 69 & None & - & - & - & Cylindrical & 6 & 5 & 182 & 8.9237 & $91 \%$ \\
\hline 70 & $\begin{array}{c}\text { None } \\
\text { Non-Lossy }\end{array}$ & - & - & - & Conical & 4 & 1 & 166 & 8.8902 & $91 \%$ \\
\hline 71 & $\begin{array}{l}\text { Conical ITO } \\
\text { Non-Lossy }\end{array}$ & 4 & 4 & 166 & None & - & - & - & 8.8755 & $90 \%$ \\
\hline 72 & $\begin{array}{l}\text { Conical ITO } \\
\text { Non-Lossy }\end{array}$ & 5 & 5 & 164 & None & - & - & - & 8.8481 & $90 \%$ \\
\hline 73 & $\begin{array}{l}\text { Cylindrical ITO } \\
\text { Non-Lossy }\end{array}$ & 3 & 2 & 132 & None & - & - & - & 8.8459 & $90 \%$ \\
\hline 74 & $\begin{array}{l}\text { Conical ITO } \\
\text { Non-Lossy }\end{array}$ & 6 & 6 & 164 & None & - & - & - & 8.8336 & $90 \%$ \\
\hline 75 & $\begin{array}{c}\text { Conical ITO } \\
\text { Lossy Cylindrical }\end{array}$ & 3 & 3 & 176 & None & - & - & - & 8.8072 & $89 \%$ \\
\hline 76 & $\begin{array}{l}\text { ITO } \\
\text { Non-Lossy }\end{array}$ & 6 & 5 & 126 & None & - & - & - & 8.7926 & $89 \%$ \\
\hline 77 & $\begin{array}{l}\text { Cylindrical ITO } \\
\text { Lossy Cylindrical }\end{array}$ & 5 & 3 & 132 & None & - & - & - & 8.7139 & $87 \%$ \\
\hline 78 & ITO & 5 & 4 & 132 & None & - & - & - & 8.6626 & $86 \%$ \\
\hline 79 & $\begin{array}{l}\text { None } \\
\text { Non-Lossy }\end{array}$ & - & - & - & Conical & 3 & 1 & 166 & 8.6015 & $85 \%$ \\
\hline 80 & Conical P-GaN & 3 & 2 & 200 & None & - & - & - & 8.5902 & $84 \%$ \\
\hline 81 & $\begin{array}{c}\text { None } \\
\text { Non-Lossy }\end{array}$ & - & - & - & Conical & 2 & 2 & 140 & 8.5884 & $84 \%$ \\
\hline 82 & $\begin{array}{c}\text { Conical ITO } \\
\text { Lossy Cylindrical }\end{array}$ & 2 & 2 & 198 & None & - & - & - & 8.5676 & $84 \%$ \\
\hline 83 & ITOO & 4 & 3 & 126 & None & - & - & - & 8.5161 & $83 \%$ \\
\hline 84 & $\begin{array}{c}\text { None } \\
\text { Non-Lossy }\end{array}$ & - & - & - & Conical & 4 & 2 & 164 & 8.5121 & $83 \%$ \\
\hline 85 & Cylindrical ITO & 4 & 2 & 132 & None & - & - & - & 8.5109 & $83 \%$ \\
\hline 86 & $\begin{array}{c}\text { None } \\
\text { Non-Lossy }\end{array}$ & - & - & - & Conical & 6 & 3 & 158 & 8.5036 & $82 \%$ \\
\hline 87 & $\begin{array}{l}\text { Conical ITO } \\
\text { Non-Lossy }\end{array}$ & 6 & 5 & 164 & None & - & - & - & 8.4914 & $82 \%$ \\
\hline 88 & $\begin{array}{l}\text { Cylindrical ITO } \\
\text { Lossy Cylindrical }\end{array}$ & 6 & 3 & 132 & None & - & - & - & 8.4904 & $82 \%$ \\
\hline 89 & $\begin{array}{c}\text { ITO } \\
\text { Non-Lossy }\end{array}$ & 6 & 4 & 132 & None & - & - & - & 8.4817 & $82 \%$ \\
\hline 90 & $\begin{array}{l}\text { Conical P-GaN } \\
\text { Non-Lossy }\end{array}$ & 6 & 5 & 200 & None & - & - & - & 8.4797 & $82 \%$ \\
\hline 91 & $\begin{array}{l}\text { Conical P-GaN } \\
\text { Non-Lossy }\end{array}$ & 6 & 4 & 200 & None & - & - & - & 8.4704 & $82 \%$ \\
\hline 92 & $\begin{array}{l}\text { Conical ITO } \\
\text { Non-Lossy }\end{array}$ & 5 & 4 & 166 & None & - & - & - & 8.4384 & $81 \%$ \\
\hline 93 & Conical P-GaN & 2 & 2 & 176 & None & - & - & - & 8.4357 & $81 \%$ \\
\hline 94 & Lossy Conical ITO & 4 & 4 & 164 & None & - & - & - & 8.4015 & $80 \%$ \\
\hline 95 & Lossy Conical ITO & 5 & 5 & 164 & None & - & - & - & 8.3905 & $80 \%$ \\
\hline 96 & Lossy Conical ITO & 6 & 6 & 164 & None & - & - & - & 8.3884 & $80 \%$ \\
\hline
\end{tabular}




\begin{tabular}{|c|c|c|c|c|c|c|c|c|c|c|}
\hline Rank & $\begin{array}{l}\text { Structure Name } \\
\text { Top Transmission }\end{array}$ & $\mu m$ & $\begin{array}{l}\mathbf{w} \\
\mu m\end{array}$ & $\begin{array}{l}\mathbf{d} \\
n m\end{array}$ & $\begin{array}{l}\text { Structure Name } \\
\text { Bottom Reflection }\end{array}$ & $\begin{array}{l}\mathbf{A} \\
\mu m\end{array}$ & $\begin{array}{l}\mathbf{w} \\
\mu m\end{array}$ & $\begin{array}{l}\mathbf{d} \\
\mathrm{nm}\end{array}$ & $\begin{array}{l}\text { Power } \\
\text { (au) }\end{array}$ & $\begin{array}{l}\text { Light Ext. } \\
\text { Eff. Imp. } \\
* *\end{array}$ \\
\hline 97 & $\begin{array}{c}\text { Non-Lossy } \\
\text { Conical P-GaN }\end{array}$ & 4 & 2 & 200 & None & - & - & - & 8.3533 & $79 \%$ \\
\hline 98 & $\begin{array}{c}\text { Lossy Conical ITO } \\
\text { Non-Lossy }\end{array}$ & 3 & 3 & 166 & None & - & - & - & 8.3495 & $79 \%$ \\
\hline 99 & $\begin{array}{l}\text { Conical P-GaN } \\
\text { Lossy Cylindrical }\end{array}$ & 5 & 4 & 200 & None & - & - & - & 8.3482 & $79 \%$ \\
\hline 100 & $\begin{array}{c}\text { ITO } \\
\text { Non-Lossy }\end{array}$ & 3 & 2 & 132 & None & - & - & - & 8.3341 & $79 \%$ \\
\hline 101 & Conical ITO & 4 & 3 & 186 & None & - & - & - & 8.2948 & $78 \%$ \\
\hline 102 & None & - & - & - & Conical & 4 & 4 & 200 & 8.26 & $77 \%$ \\
\hline 103 & $\begin{array}{c}\text { None } \\
\text { Non-Lossy }\end{array}$ & - & - & - & Conical & 5 & 3 & 168 & 8.2255 & $76 \%$ \\
\hline 104 & $\begin{array}{l}\text { Conical P-GaN } \\
\text { Lossy Cylindrical }\end{array}$ & 5 & 5 & 200 & None & - & - & - & 8.2178 & $76 \%$ \\
\hline 105 & $\begin{array}{c}\text { ITO } \\
\text { Non-Lossy }\end{array}$ & 5 & 3 & 132 & None & - & - & - & 8.2023 & $76 \%$ \\
\hline 106 & $\begin{array}{l}\text { Conical P-GaN } \\
\text { Non-Lossy }\end{array}$ & 6 & 2 & 180 & None & - & - & - & 8.1932 & $76 \%$ \\
\hline 107 & Conical ITO & 6 & 4 & 166 & None & - & - & - & 8.1511 & $75 \%$ \\
\hline 108 & Lossy Conical ITO & 2 & 2 & 198 & None & - & - & - & 8.1446 & $75 \%$ \\
\hline 109 & $\begin{array}{c}\text { None } \\
\text { Non-Lossy }\end{array}$ & - & - & - & Conical & 6 & 4 & 166 & 8.1266 & $74 \%$ \\
\hline 110 & Conical P-GaN & 5 & 2 & 150 & None & - & - & - & 8.112 & $74 \%$ \\
\hline 111 & None & - & - & - & Conical & 4 & 3 & 168 & 8.106 & $74 \%$ \\
\hline 112 & $\begin{array}{c}\text { Lossy Conical ITO } \\
\text { Non-Lossy }\end{array}$ & 6 & 5 & 164 & None & - & - & - & 8.0851 & $73 \%$ \\
\hline 113 & Conical P-GaN & 6 & 1 & 84 & None & - & - & - & 8.068 & $73 \%$ \\
\hline 114 & $\begin{array}{c}\text { None } \\
\text { Lossy Cylindrical }\end{array}$ & - & - & - & Conical & 5 & 5 & 198 & 8.0417 & $73 \%$ \\
\hline 115 & ITO & 6 & 3 & 132 & None & - & - & - & 8.0294 & $72 \%$ \\
\hline 116 & $\begin{array}{l}\text { Lossy Conical ITO } \\
\text { Lossy Cylindrical }\end{array}$ & 5 & 4 & 166 & None & - & - & - & 8.0293 & $72 \%$ \\
\hline 117 & $\begin{array}{l}\text { ITO } \\
\text { Non-Lossy }\end{array}$ & 4 & 2 & 132 & None & - & - & - & 7.9922 & $71 \%$ \\
\hline 118 & $\begin{array}{l}\text { Conical ITO } \\
\text { Non-Lossy }\end{array}$ & 5 & 3 & 196 & None & - & - & - & 7.9811 & $71 \%$ \\
\hline 119 & $\begin{array}{c}\text { Cylindrical ITO } \\
\text { Non-Lossy }\end{array}$ & 5 & 2 & 132 & None & - & - & - & 7.9619 & $71 \%$ \\
\hline 120 & Conical ITO & 3 & 2 & 198 & None & - & - & - & 7.9523 & $71 \%$ \\
\hline 121 & $\begin{array}{c}\text { None } \\
\text { Non-Lossy }\end{array}$ & - & - & - & Conical & 3 & 2 & 166 & 7.9435 & $70 \%$ \\
\hline 122 & $\begin{array}{l}\text { Conical P-GaN } \\
\text { Non-Lossy }\end{array}$ & 6 & 6 & 200 & None & - & - & - & 7.9382 & $70 \%$ \\
\hline 123 & Conical ITO & 1 & 1 & 66 & None & - & - & - & 7.9338 & $70 \%$ \\
\hline 124 & $\begin{array}{c}\text { Lossy Conical ITO } \\
\text { Non-Lossy }\end{array}$ & 4 & 3 & 176 & None & - & - & - & 7.8874 & $69 \%$ \\
\hline 125 & $\begin{array}{l}\text { Cylindrical ITO } \\
\text { Non-Lossy }\end{array}$ & 6 & 2 & 132 & None & - & - & - & 7.8611 & $69 \%$ \\
\hline 126 & Conical P-GaN & 4 & 4 & 200 & None & - & - & - & 7.839 & $68 \%$ \\
\hline 127 & None & - & - & - & Conical & 5 & 4 & 168 & 7.8285 & $68 \%$ \\
\hline 128 & $\begin{array}{c}\text { Lossy Conical ITO } \\
\text { Non-Lossy }\end{array}$ & 6 & 4 & 166 & None & - & - & - & 7.7838 & $67 \%$ \\
\hline 129 & Cylindrical ITO & 3 & 1 & 132 & None & - & - & - & 7.7389 & $66 \%$ \\
\hline
\end{tabular}




\begin{tabular}{|c|c|c|c|c|c|c|c|c|c|c|}
\hline Rank & $\begin{array}{l}\text { Structure Name } \\
\text { Top Transmission }\end{array}$ & $\begin{array}{l}\mathbf{A} \\
\mu m\end{array}$ & $\begin{array}{l}\mathbf{w} \\
\mu m\end{array}$ & $\begin{array}{l}\mathbf{d} \\
n m\end{array}$ & $\begin{array}{l}\text { Structure Name } \\
\text { Bottom Reflection }\end{array}$ & $\begin{array}{l}\mathbf{A} \\
\mu m\end{array}$ & $\begin{array}{l}\mathbf{w} \\
\mu m\end{array}$ & $\begin{array}{l}\mathbf{d} \\
n m\end{array}$ & $\begin{array}{l}\text { Power } \\
\text { (au) }\end{array}$ & $\begin{array}{l}\text { Light Ext. } \\
\text { Eff. Imp. } \\
* *\end{array}$ \\
\hline 130 & $\begin{array}{l}\text { Non-Lossy } \\
\text { Conical ITO }\end{array}$ & 6 & 3 & 198 & None & - & - & - & 7.7322 & $66 \%$ \\
\hline 131 & $\begin{array}{c}\text { None } \\
\text { Non-Lossy }\end{array}$ & - & - & - & Conical & 6 & 5 & 166 & 7.6904 & $65 \%$ \\
\hline 132 & $\begin{array}{c}\text { Conical P-GaN } \\
\text { Non-Lossy }\end{array}$ & 5 & 3 & 200 & None & - & - & - & 7.6663 & $64 \%$ \\
\hline 133 & Conical P-GaN & 6 & 3 & 200 & None & - & - & - & 7.6398 & $64 \%$ \\
\hline 134 & Lossy Conical ITO & 5 & 3 & 186 & None & - & - & - & 7.6127 & $63 \%$ \\
\hline 135 & $\begin{array}{l}\text { Lossy Conical ITO } \\
\text { Lossy Cylindrical }\end{array}$ & 3 & 2 & 198 & None & - & - & - & 7.5777 & $63 \%$ \\
\hline 136 & $\begin{array}{l}\text { ITO } \\
\text { Lossy Cylindrical }\end{array}$ & 5 & 2 & 132 & None & - & - & - & 7.5773 & $63 \%$ \\
\hline 137 & $\begin{array}{l}\text { ITO } \\
\text { Non-Lossy }\end{array}$ & 6 & 2 & 132 & None & - & - & - & 7.4957 & $61 \%$ \\
\hline 138 & Conical ITO & 4 & 2 & 200 & None & - & - & - & 7.4838 & $61 \%$ \\
\hline 139 & $\begin{array}{c}\text { None } \\
\text { Non-Lossy }\end{array}$ & - & - & - & Conical & 2 & 1 & 176 & 7.4801 & $60 \%$ \\
\hline 140 & Cylindrical ITO & 2 & 1 & 132 & None & - & - & - & 7.4782 & $60 \%$ \\
\hline 141 & Lossy Conical ITO & 1 & 1 & 200 & None & - & - & - & 7.4199 & $59 \%$ \\
\hline 142 & $\begin{array}{l}\text { Lossy Conical ITO } \\
\text { Lossy Cylindrical }\end{array}$ & 6 & 3 & 198 & None & - & - & - & 7.4017 & $59 \%$ \\
\hline 143 & $\begin{array}{l}\text { ITO } \\
\text { Lossy Cylindrical }\end{array}$ & 3 & 1 & 132 & None & - & - & - & 7.3744 & $58 \%$ \\
\hline 144 & $\begin{array}{l}\text { ITO } \\
\text { Non-Lossy }\end{array}$ & 2 & 1 & 132 & None & - & - & - & 7.2745 & $56 \%$ \\
\hline 145 & $\begin{array}{l}\text { Conical P-GaN } \\
\text { Non-Lossy }\end{array}$ & 4 & 3 & 200 & None & - & - & - & 7.2347 & $55 \%$ \\
\hline 146 & Conical ITO & 5 & 2 & 200 & None & - & - & - & 7.2206 & $55 \%$ \\
\hline 147 & Lossy Conical ITO & 4 & 2 & 198 & None & - & - & - & 7.2135 & $55 \%$ \\
\hline 148 & $\begin{array}{l}\text { None } \\
\text { Non-Lossy }\end{array}$ & - & - & - & Conical & 6 & 6 & 182 & 7.206 & $55 \%$ \\
\hline 149 & $\begin{array}{l}\text { Conical ITO } \\
\text { Non-Lossy }\end{array}$ & 6 & 2 & 200 & None & - & - & - & 7.1097 & $53 \%$ \\
\hline 150 & Cylindrical ITO & 4 & 1 & 132 & None & - & - & - & 7.0569 & $51 \%$ \\
\hline 151 & $\begin{array}{c}\text { Lossy Conical ITO } \\
\text { Non-Lossy }\end{array}$ & 5 & 2 & 200 & None & - & - & - & 6.9781 & $50 \%$ \\
\hline 152 & Cylindrical ITO & 5 & 1 & 132 & None & - & - & - & 6.8962 & $48 \%$ \\
\hline 153 & $\begin{array}{l}\text { Lossy Conical ITO } \\
\text { Lossy Cylindrical }\end{array}$ & 6 & 2 & 200 & None & - & - & - & 6.8607 & $47 \%$ \\
\hline 154 & $\begin{array}{l}\text { ITO } \\
\text { Non-Lossy }\end{array}$ & 4 & 1 & 132 & None & - & - & - & 6.8469 & $47 \%$ \\
\hline 155 & $\begin{array}{l}\text { Conical ITO } \\
\text { Non-Lossy }\end{array}$ & 3 & 1 & 106 & None & - & - & - & 6.7853 & $46 \%$ \\
\hline 156 & $\begin{array}{l}\text { Cylindrical ITO } \\
\text { Lossy Cylindrical }\end{array}$ & 6 & 1 & 160 & None & - & - & - & 6.7496 & $45 \%$ \\
\hline 157 & $\begin{array}{l}\text { ITO } \\
\text { Non-Lossy }\end{array}$ & 5 & 1 & 132 & None & - & - & - & 6.6911 & $44 \%$ \\
\hline 158 & Conical ITO & 2 & 1 & 98 & None & - & - & - & 6.6437 & $43 \%$ \\
\hline 159 & $\begin{array}{l}\text { Lossy Conical ITO } \\
\text { Lossy Cylindrical }\end{array}$ & 4 & 4 & 164 & Cylindrical & 2 & 1 & 136 & 6.6237 & $42 \%$ \\
\hline 160 & ITO & 6 & 1 & 132 & None & - & - & - & 6.568 & $41 \%$ \\
\hline 161 & $\begin{array}{c}\text { Lossy Conical ITO } \\
\text { Non-Lossy }\end{array}$ & 3 & 1 & 98 & None & - & - & - & 6.5331 & $40 \%$ \\
\hline 162 & Conical P-GaN & 3 & 3 & 50 & None & - & - & - & 6.4846 & $39 \%$ \\
\hline
\end{tabular}




\begin{tabular}{|c|c|c|c|c|c|c|c|c|c|c|}
\hline Rank & $\begin{array}{l}\text { Structure Name } \\
\text { Top Transmission }\end{array}$ & $\begin{array}{l}\mathbf{A} \\
\mu m\end{array}$ & $\begin{array}{l}\mathbf{w} \\
\mu m\end{array}$ & $\begin{array}{l}\mathbf{d} \\
n m\end{array}$ & $\begin{array}{l}\text { Structure Name } \\
\text { Bottom Reflection }\end{array}$ & $\begin{array}{l}\mathbf{A} \\
\mu m\end{array}$ & $\begin{array}{l}\mathbf{w} \\
\mu m\end{array}$ & $\begin{array}{l}\mathbf{d} \\
n m\end{array}$ & $\begin{array}{l}\text { Power } \\
\text { (au) }\end{array}$ & $\begin{array}{l}\text { Light Ext. } \\
\text { Eff. Imp. } \\
* *\end{array}$ \\
\hline 163 & $\begin{array}{l}\text { Non-Lossy } \\
\text { Conical ITO }\end{array}$ & 4 & 1 & 132 & None & - & - & - & 6.4786 & $39 \%$ \\
\hline 164 & $\begin{array}{c}\text { Lossy Conical ITO } \\
\text { Non-Lossy }\end{array}$ & 2 & 1 & 98 & None & - & - & - & 6.4549 & $38 \%$ \\
\hline 165 & Conical ITO & 6 & 1 & 164 & None & - & - & - & 6.3414 & $36 \%$ \\
\hline 166 & $\begin{array}{c}\text { Lossy Conical ITO } \\
\text { Non-Lossy }\end{array}$ & 4 & 1 & 132 & None & - & - & - & 6.2865 & $35 \%$ \\
\hline 167 & $\begin{array}{l}\text { Conical ITO } \\
\text { Non-Lossy }\end{array}$ & 5 & 1 & 198 & None & - & - & - & 6.2306 & $34 \%$ \\
\hline 168 & Conical P-GaN & 1 & 1 & 138 & Cylindrical & 2 & 1 & 136 & 6.2106 & $33 \%$ \\
\hline 169 & Lossy Conical ITO & 6 & 1 & 164 & None & - & - & - & 6.1995 & $33 \%$ \\
\hline 170 & None & - & - & - & Conical & 3 & 3 & 72 & 6.1618 & $32 \%$ \\
\hline 171 & $\begin{array}{l}\text { Lossy Conical ITO } \\
\text { Non-Lossy }\end{array}$ & 5 & 1 & 164 & None & - & - & - & 6.1565 & $32 \%$ \\
\hline 172 & $\begin{array}{l}\text { Conical ITO } \\
\text { Non-Lossy }\end{array}$ & 4 & 4 & 166 & Cylindrical & 2 & 1 & 136 & 6.1401 & $32 \%$ \\
\hline 173 & $\begin{array}{c}\text { Cylindrical P-GaN } \\
\text { Non-Lossy }\end{array}$ & 2 & 1 & 156 & Cylindrical & 2 & 1 & 136 & 6.1368 & $32 \%$ \\
\hline 174 & $\begin{array}{l}\text { Cylindrical ITO } \\
\text { Lossy Cylindrical }\end{array}$ & 1 & 1 & 126 & Cylindrical & 2 & 1 & 136 & 5.5491 & $19 \%$ \\
\hline 175 & $\begin{array}{l}\text { ITO } \\
\text { Non-Lossy }\end{array}$ & 1 & 1 & 186 & Cylindrical & 2 & 1 & 136 & 5.5181 & $18 \%$ \\
\hline 176 & Conical ITO & 4 & 4 & 166 & Conical & 1 & 1 & 200 & 5.4614 & $17 \%$ \\
\hline 177 & $\begin{array}{c}\text { Lossy Conical ITO } \\
\text { Non-Lossy }\end{array}$ & 4 & 4 & 164 & Conical & 1 & 1 & 200 & 5.3947 & $16 \%$ \\
\hline 178 & $\begin{array}{c}\text { Conical P-GaN } \\
\text { Non-Lossy }\end{array}$ & 1 & 1 & 138 & Conical & 1 & 1 & 200 & 5.2641 & $13 \%$ \\
\hline 179 & $\begin{array}{c}\text { Cylindrical P-GaN } \\
\text { Non-Lossy }\end{array}$ & 2 & 1 & 156 & Conical & 1 & 1 & 200 & 5.2185 & $12 \%$ \\
\hline 180 & $\begin{array}{l}\text { Cylindrical ITO } \\
\text { Lossy Cylindrical }\end{array}$ & 1 & 1 & 126 & Conical & 1 & 1 & 200 & 5.0291 & $8 \%$ \\
\hline 181 & ITOO & 1 & 1 & 126 & Conical & 1 & 1 & 200 & 4.8967 & $5 \%$ \\
\hline 182 & $\begin{array}{c}\text { None }^{2} \\
\text { Lossy Cylindrical }\end{array}$ & - & - & - & None & - & - & - & 4.6608 & $0 \%$ \\
\hline 183 & ITO & 1 & 1 & 186 & Conical & 1 & 1 & 200 & 4.2404 & $-9 \%$ \\
\hline
\end{tabular}

* Averaged between two adjacent grating heights data points due to two peak points

** Meant to read "Light Extraction Efficiency Improvement"

${ }^{1}$ This grating is the partially optimized case of case 1 for the reflection-transmission gratings

${ }^{2}$ This is the control case (no grating) 


\section{APPENDIX B: RSOFT_TOOLS SOURCE CODE}

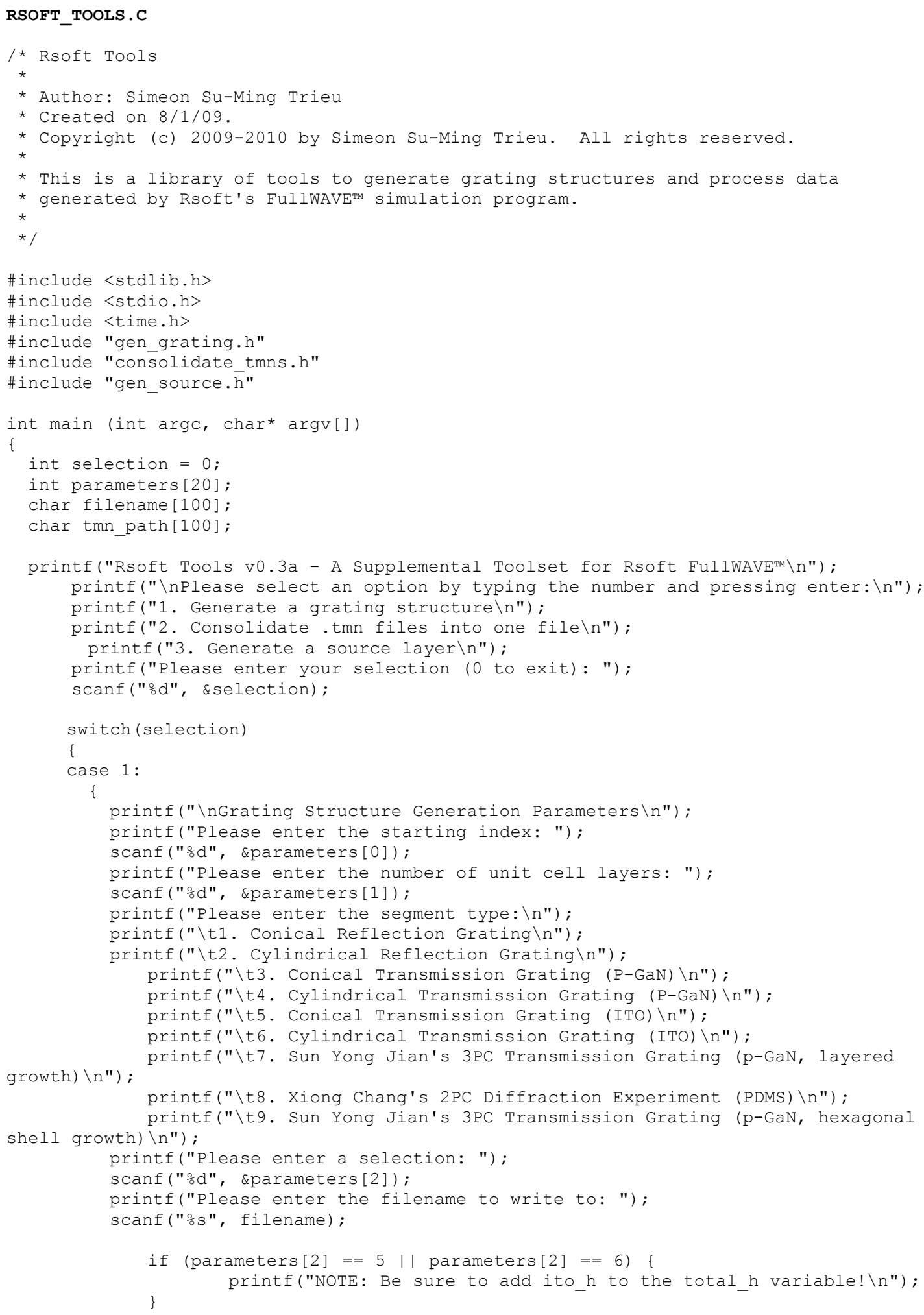




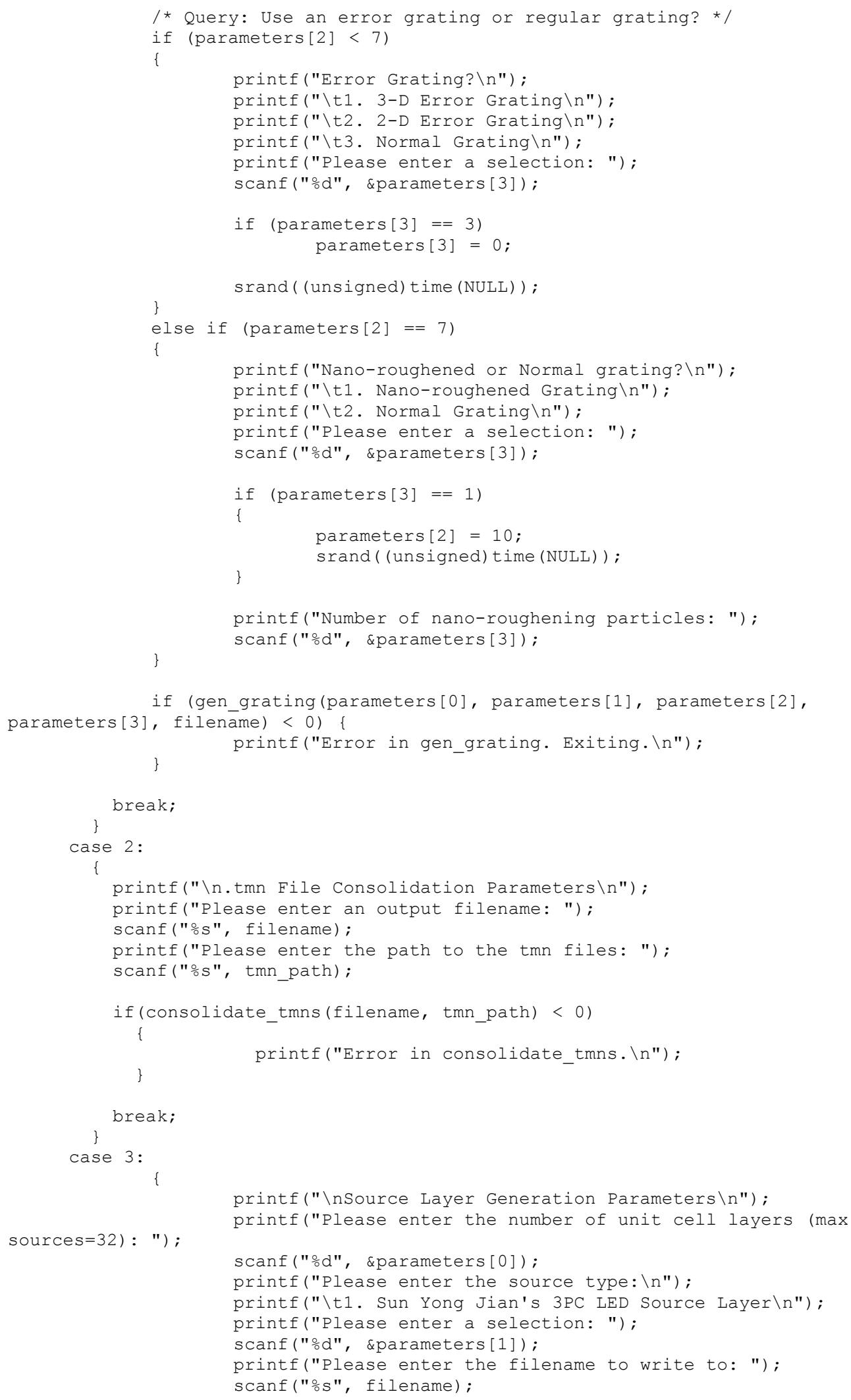




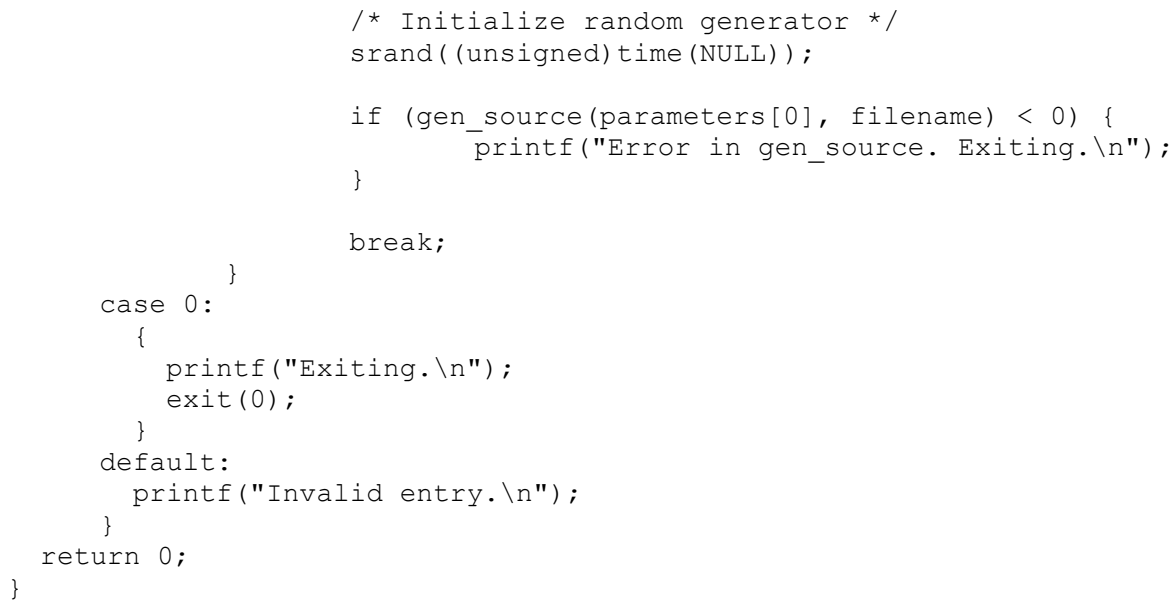

GEN_SOURCE.C

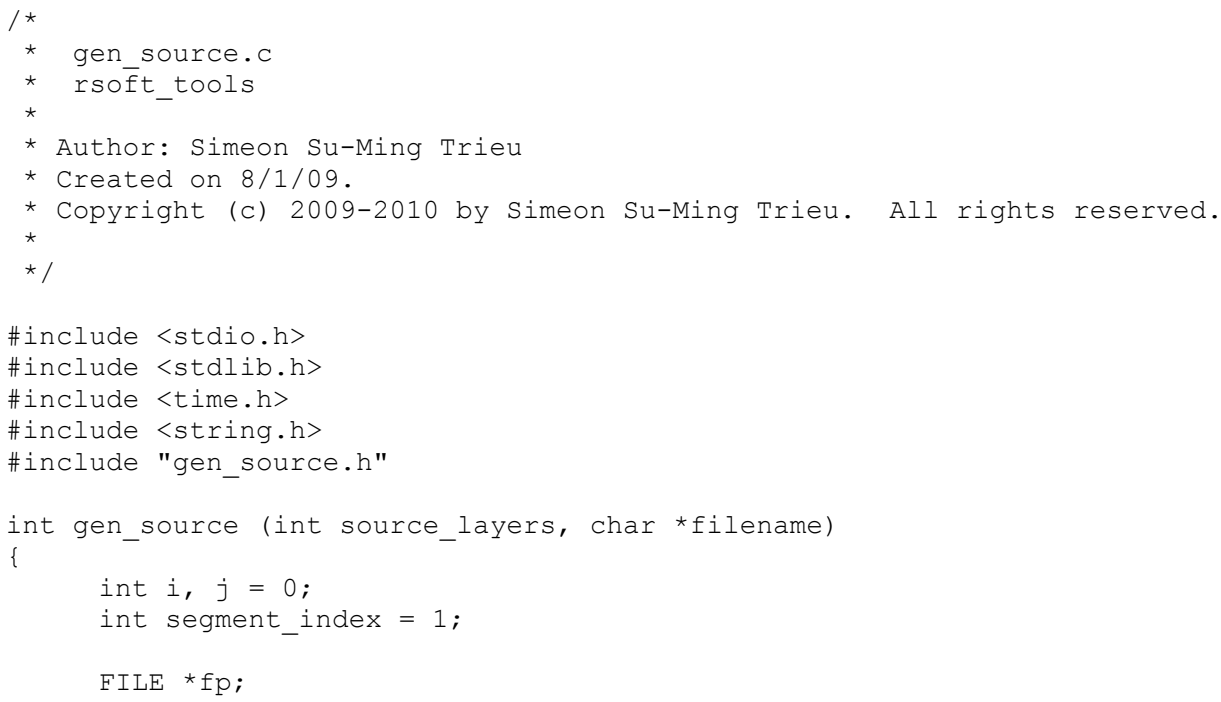




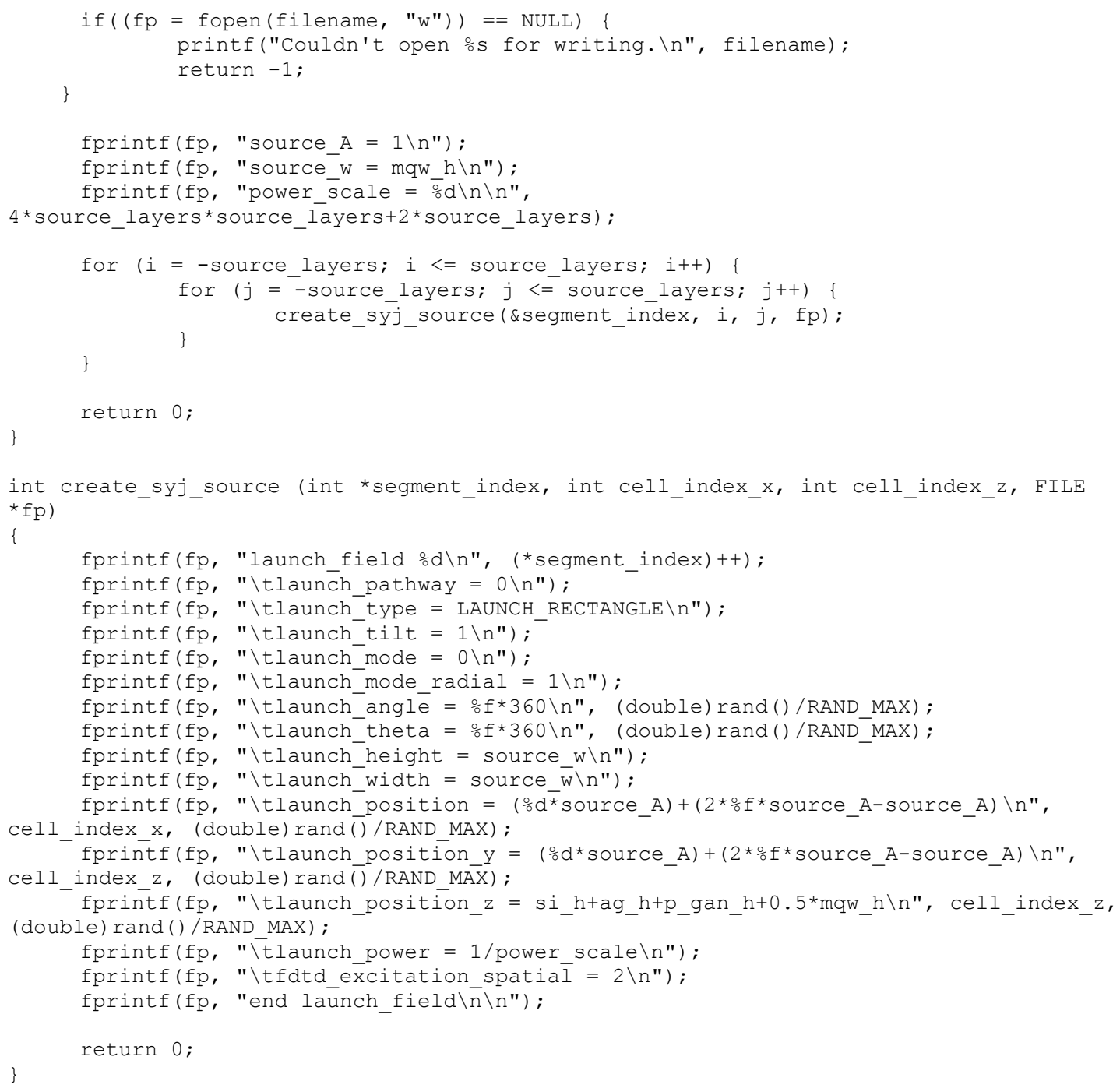

GEN_GRATING.H

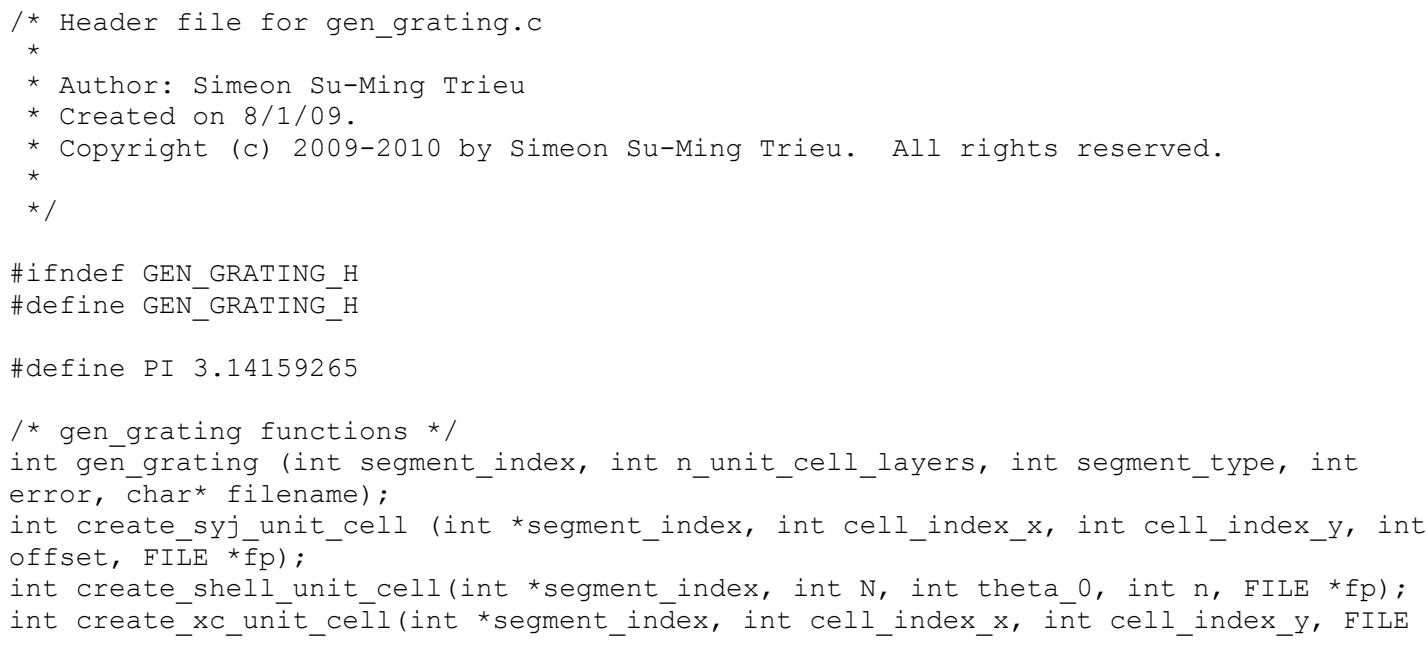




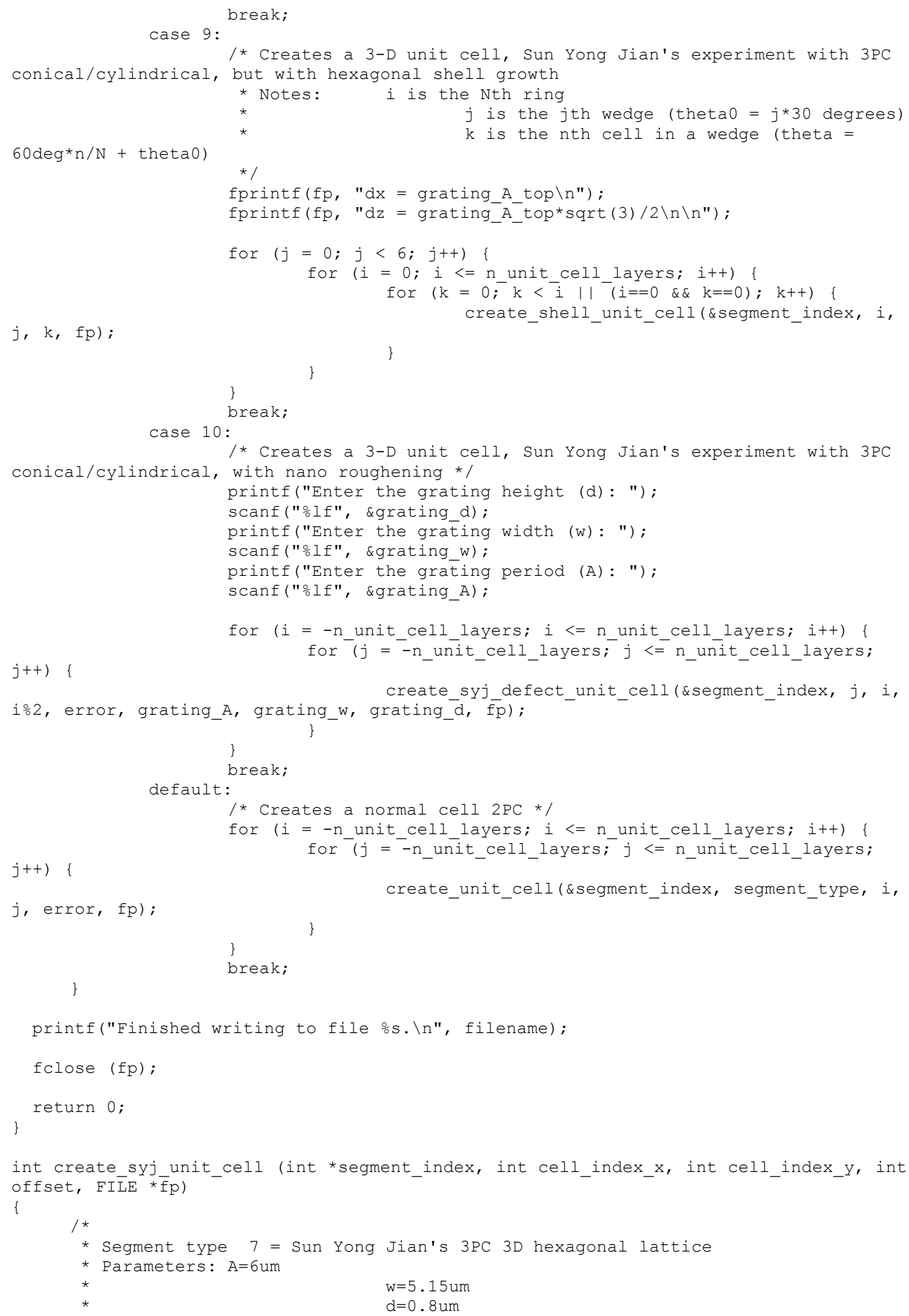




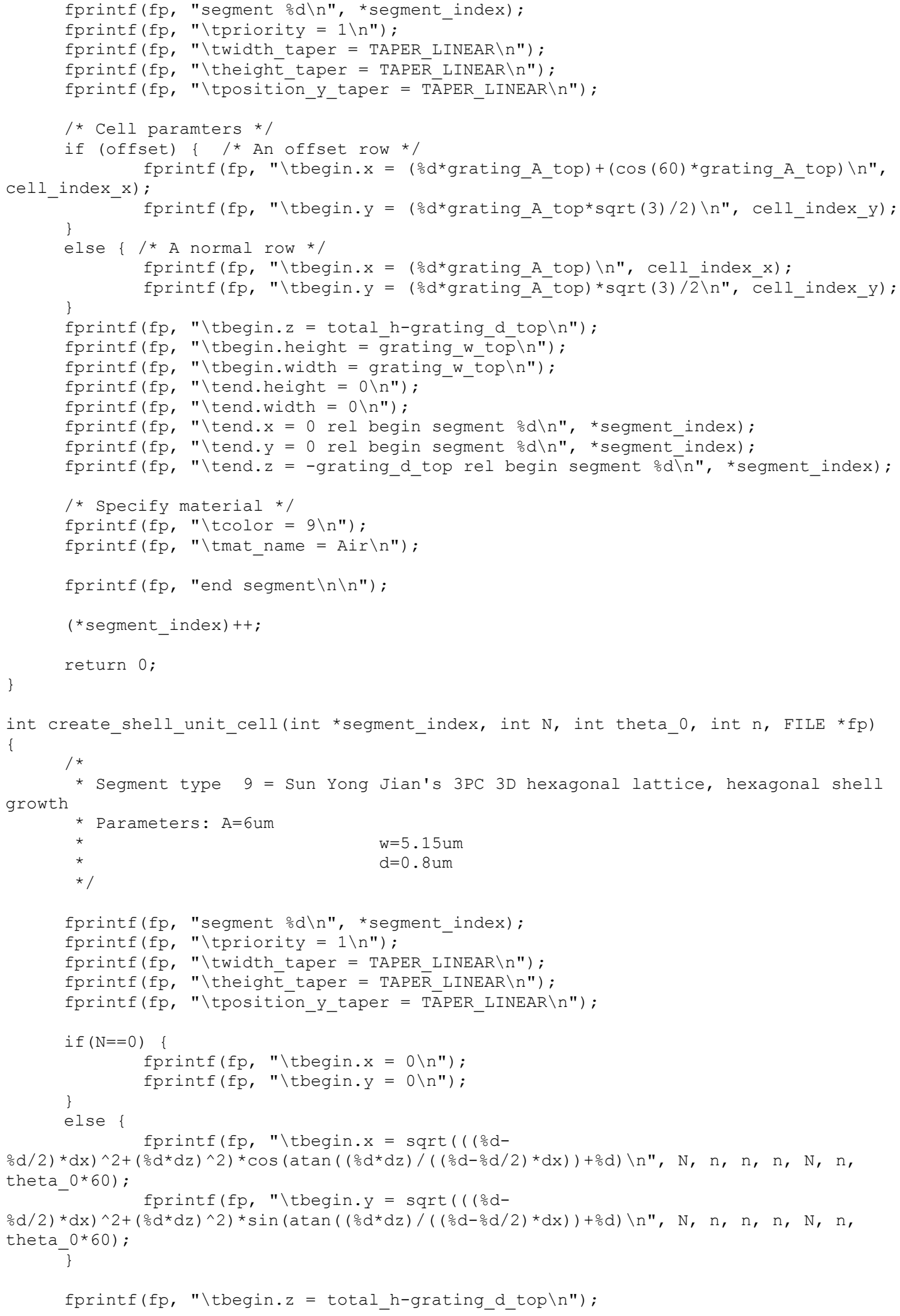




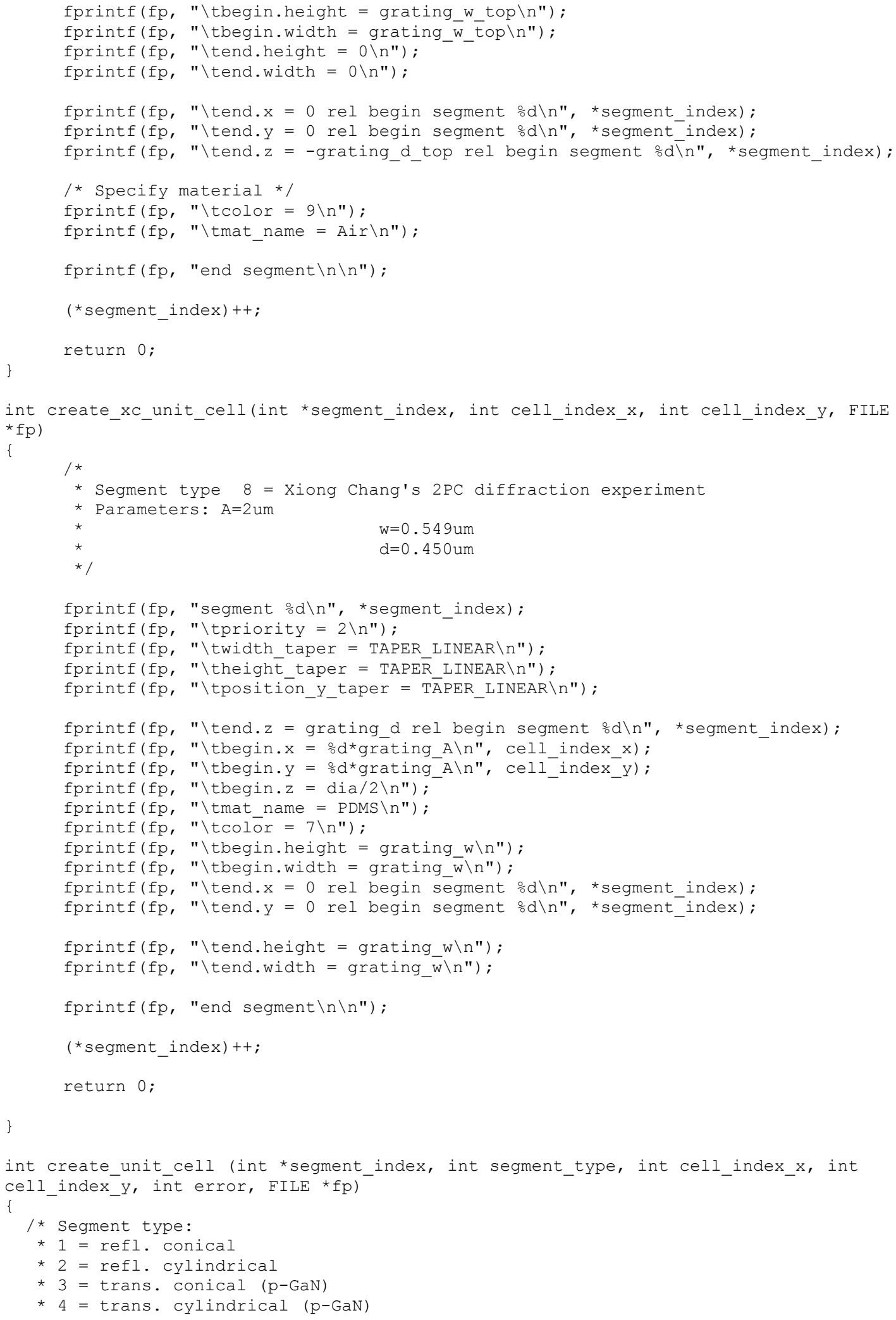


* 5 = trans. conical (ITO)

* 6 = trans. cylindrical (ITO)

* ...

$\star 1$

/* Random number generator setup */

fprintf(fp, "segment od \n", *segment_index) ;

fprintf(fp, "\tpriority $=1 \backslash \mathrm{n} "$ ) ;

fprintf (fp, "\twidth taper = TAPER LINEAR \n");

fprintf (fp, "\theight taper = TAPER_LINEAR \n") ;

fprintf(fp, "\tposition_y_taper = TĀPER_LINEAR $\backslash n "$ ) ;

if (segment type > 2) \{ / A top transmission grating */

if (error $>0)\{\quad / * A$ 2-D or 3-D error grating */

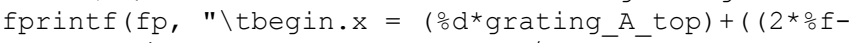

1 ) ${ }^{*}{ }^{*}$ grating $A$ top) $\backslash n "$, cell index $x$, (double) rand()/RAND $\overline{M A X}$ ).

if $($ error $==\overline{2}) / * A 2-D$ error grating */

fprintf(fp, "\tbegin.y $=$ o d*grating_A_top $\backslash n "$,

cell_index_y);

else /* 3-D grating */

fprintf (fp, "\tbegin.y $=\left(\% \mathrm{~d}^{*}\right.$ grating_A_top $)+((2 * \% f-$

1) ${ }^{*}$ *grating_A_top) \n", cell_index_y, (double) rand ()/RAND_MAX) ;

\}

else $\{/$ * A normal grating */

fprintf(fp, "\tbegin.x = (\%d*grating A top) \n", cell index $x)$;

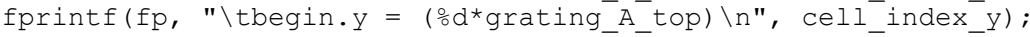

fprintf(fp, "\tbegin.z = total_h-grating_d_top $\backslash n "$ );

fprintf(fp, "\tbegin.height = grating_w_top $\backslash n "$ );

fprintf(fp, "\tbegin.width = grating_w_top $\backslash \mathrm{n} "$ );

fprintf(fp, "\tend. $z=$ grating_d_top rel begin segment $\% d \backslash n "$,

* segment index):

if (segment type $<=4)\{/ * \mathrm{P}-\mathrm{GaN} * /$

fprintf(fp, "\tcolor $=9 \backslash \mathrm{n} "$ );

fprintf(fp, "\tmat_name $=\mathrm{GaN} \backslash \mathrm{n} "$ ) ;

else $\{/ *$ ITO */

fprintf (fp, "\tcolor $=7 \backslash \mathrm{n} ")$;

\}

fprintf(fp, "\tmat_name = ITO $\backslash n ")$;

else $\{/ *$ A bottom reflection grating */

fprintf(fp, "\tend. $z=$ grating_d rel begin segment $\% d \backslash n "$,

*segment_index);

if (error > 1) \{ $/ *$ A error grating */

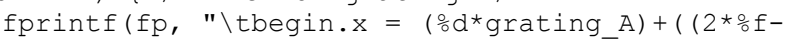

$1){ }^{*}{ }^{*}$ grating $\left.A\right) \backslash \mathrm{n} "$, cell index $\mathrm{x}$, (double) rand ()/RAND MAX) ;

if (error $==2)$

else

fprintf(fp, "\tbegin.y = od*grating_A \n", cell_index_y) ;

fprintf (fp, " $\backslash$ tbegin.y $=\left(\% d^{*} g r a t i n g \_A\right)+((2 * \% f-$

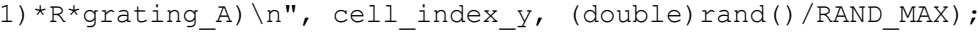

\}

else \{ / * A normal grating */

fprintf(fp, "\tbegin.x = od*grating_A \n", cell_index_x);

\} fprintf(fp, "\tbegin.y = od*grating $A \backslash n^{*}$, cell $^{-}$index ${ }^{-}$) ;

fprintf(fp, "\tbegin.z = si $h+a g h \backslash n ")$;

fprintf(fp, "\tmat name $=A \bar{g} \backslash n ")$;

fprintf (fp, "\tcolor $=14 \backslash \mathrm{n} ")$;

fprintf(fp, "\tbegin.height = grating_w $\backslash n ")$;

fprintf(fp, "\tbegin.width = grating_ $\bar{w} \backslash \mathrm{n} ")$;

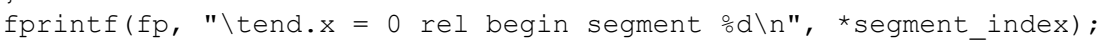

fprintf(fp, "\tend.y = 0 rel begin segment od \n", *segment_index); 


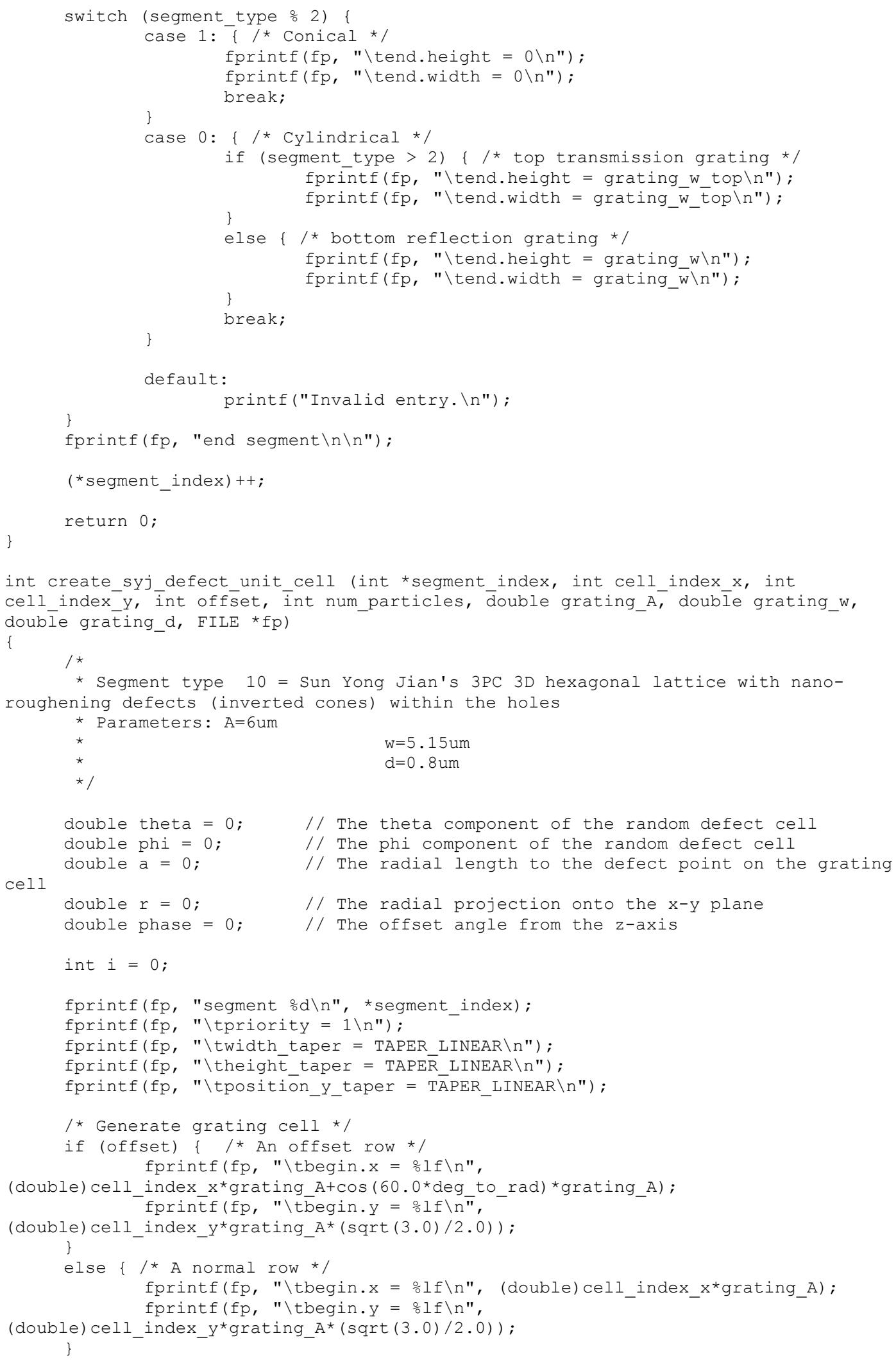




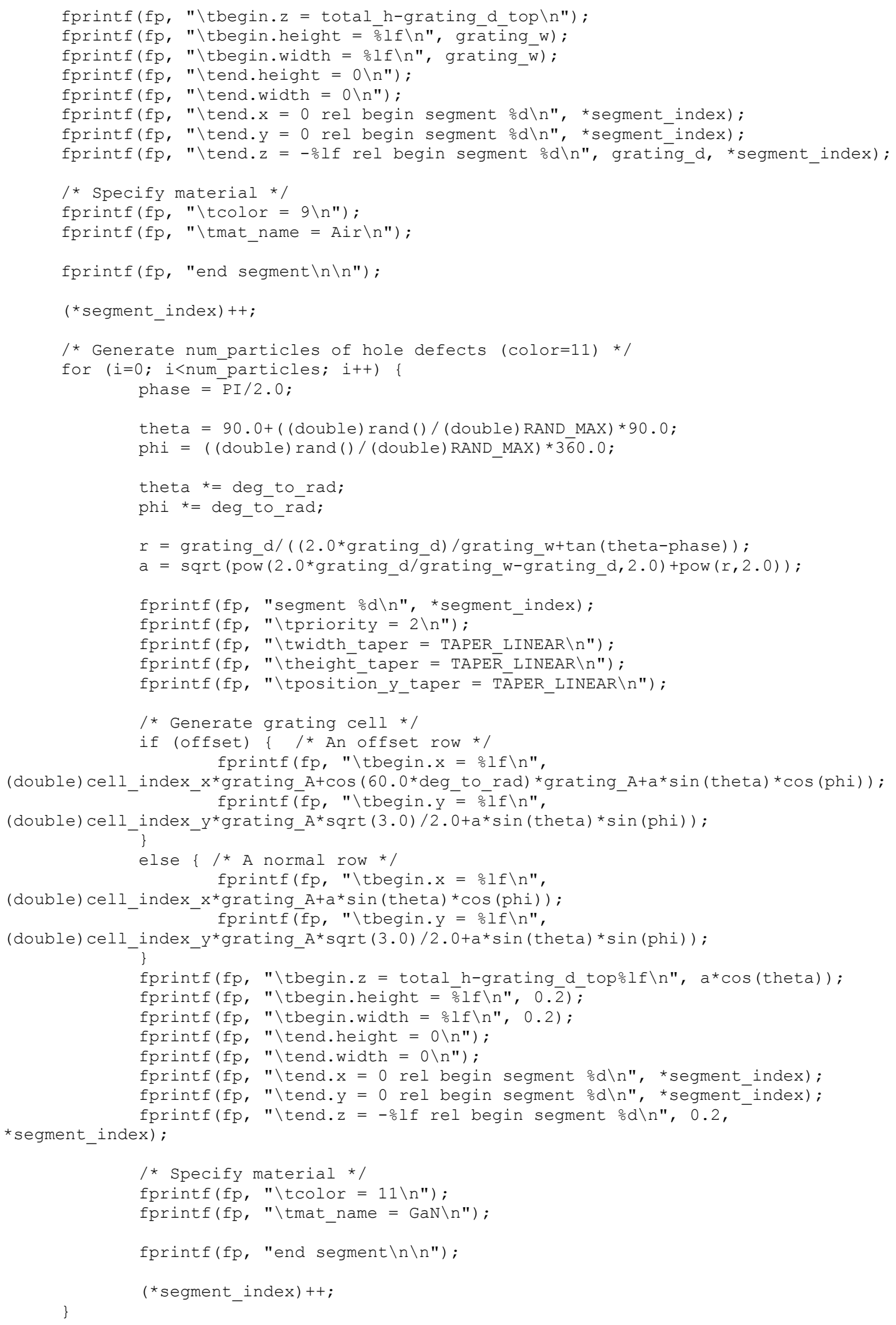




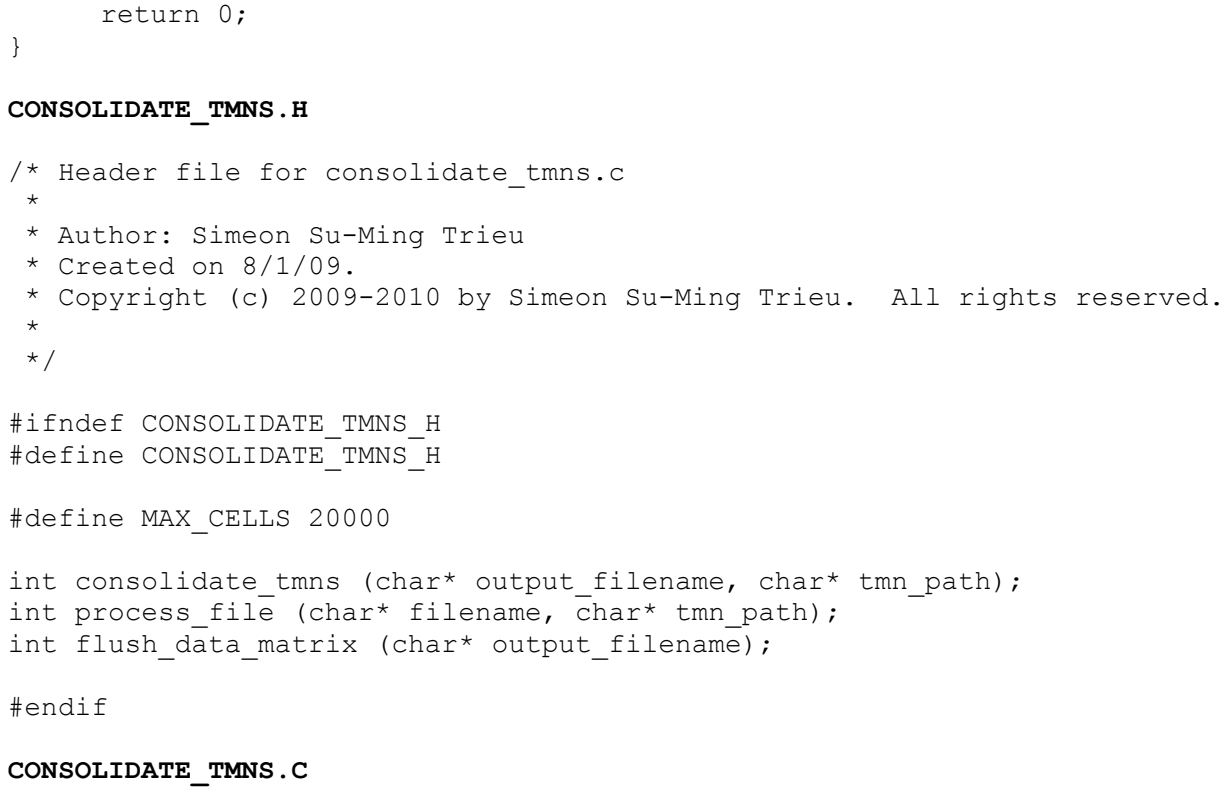




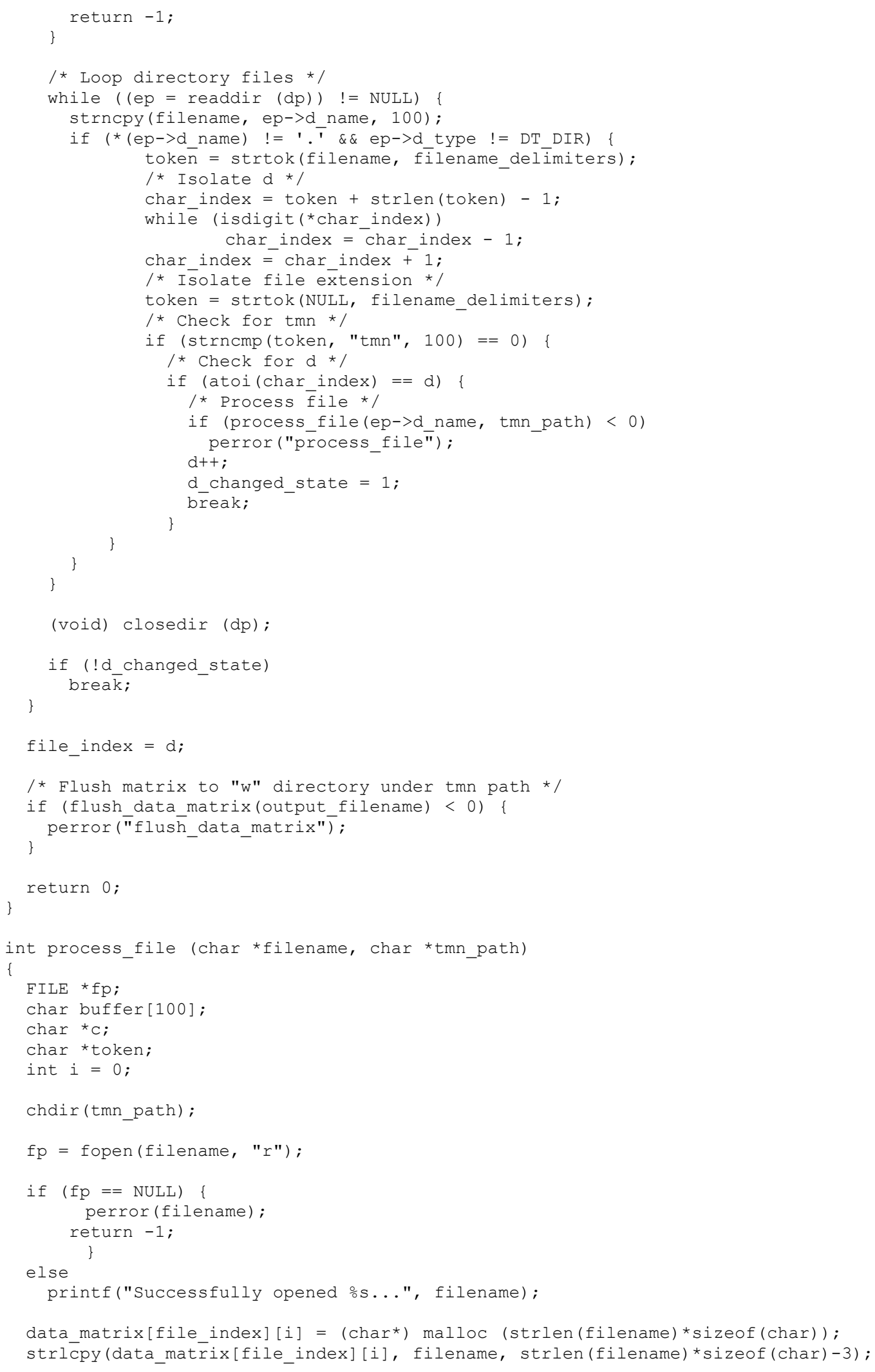




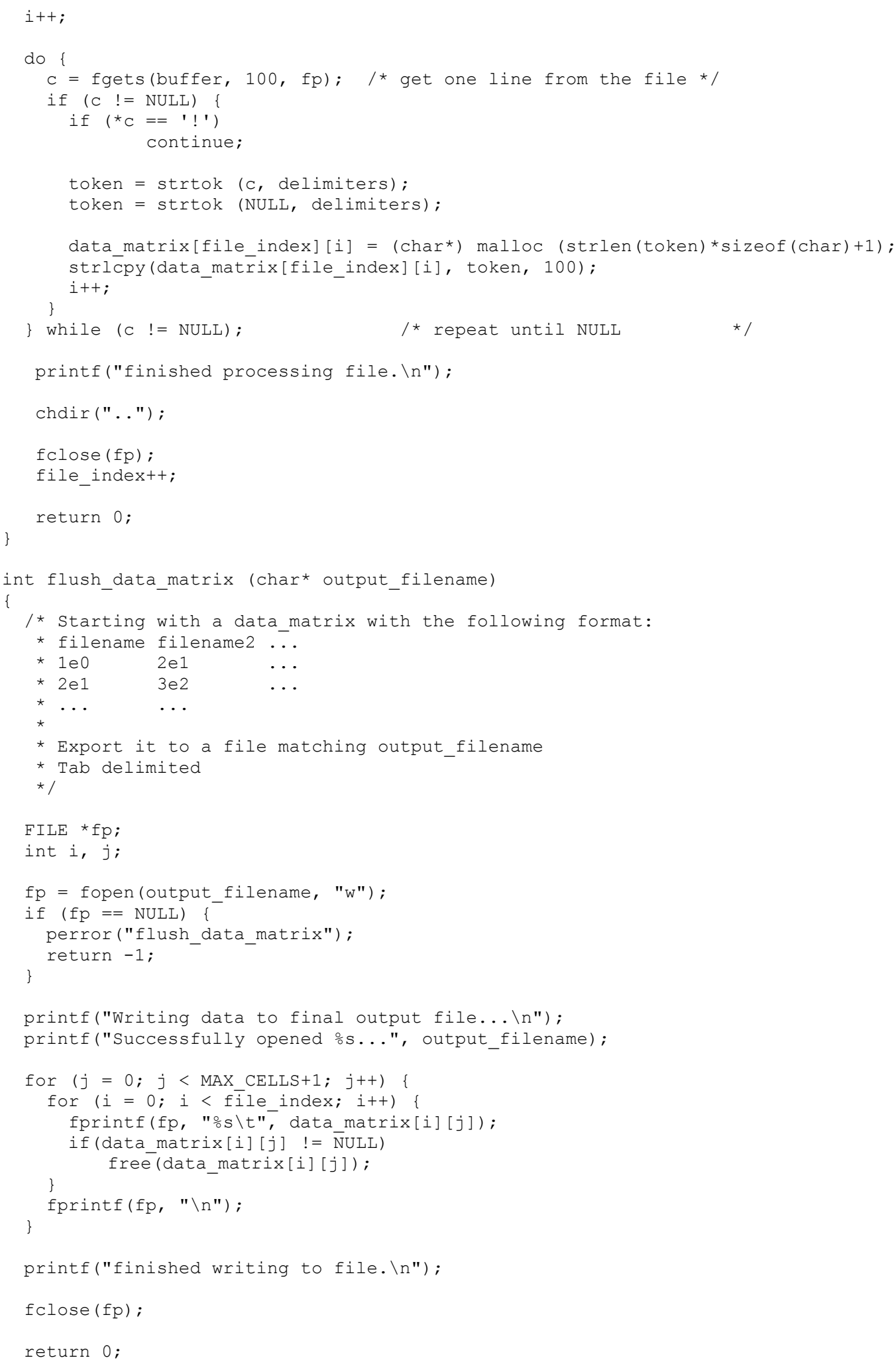




\section{PUBLISHED WORKS}

[1] S. Trieu and X. Jin, "Study of Top and Bottom Photonic Gratings on GaN LED with Error Grating Models,” IEEE J. Quantum Electron., Feb 2010.

[2] S. Trieu, X. Jin, C. Xiong, X. X. Fu, X. Kang, G. Y. Zhang, B. Zhang, F. Wang, "Simulation and Experiment on 2PC Transmitted Diffraction Grating for GaN LEDs" Nanotech. 2010, June 2010, Anaheim, CA.

[3] S. Trieu, X. Jin, C. Xiong, X. X. Fu, X. N. Kang, G. Y. Zhang and B. Zhang, "Simulation of Three-fold Symmetric Photonic Crystal Structures on Top of GaN LEDs”, CLEO, May 2010, San Jose, CA.

[4] S. Trieu, X. Jin, B. Zhang, T. Dai, K. Bao, X. N. Kang and G. Y.Zhang, "Light Extraction Improvement of GaN-based Light-emitting Diodes using Patterned Undoped GaN Bottom Reflection Gratings”, SPIE Proceedings Vol. 7216, Photonics West, San Jose, CA USA 24-29, January 2009.

[5] X. Jin, S. Jobe, S. Trieu, B. Husain, J. Flickinger, T. Dai, B. Zhang, X.-N. Kang, and G.Y. Zhang, "Mode Pattern Analysis of Gallium Nitride-based Laser diodes", SPIE Proceedings Vol. 7382, ISPDI 2009, Beijing, China, June, 2009.

[6] X. Jin, S. Trieu, Fei Wang, B. Zhang, T. Dai, X. N. Kang, and G. Y. Zhang, "Design Simulation of Top ITO Gratings to Improve Light Transmission for Gallium Nitride LEDs”, ITNG2009, Las Vegas, Nevada, USA, April 27-29, 2009.

[7] X. Jin, D. Derickson, S. Trieu, and S. O. Agbo, "Photonics Research and Education at California Polytechnic State University”, ASEE/PSW 2009 San Diego, CA, March 19-20, 2009.

[8] X. Jin, B. Zhang, T. Dai, W. Wei, X.-N. Kang, G.-Y. Zhang, S. Trieu, and F. Wang, “Optimization of Top Polymer Gratings to Improve GaN LEDs Light 
Transmission”, Chinese Optics letter, vol.6, 2008.

[9] X. Jin and S. Trieu, "Improvement of light transmission using photonic lattices for solar-cells," Solar Energy 2008, Stanford, California, June 2008. 


\section{REFERENCES}

[1] O. Svensk, P. T. Torma, S. Suihkonen, M. Ali, H. Lipsanen, M. Sopanen, A. Odnoblyudov, V. E. Bougrov, "Enhanced electroluminescence in $405 \mathrm{~nm}$ InGaN/GaN LEDs by optimized electron blocking layer,’ J. of Crystal Growth, vol. 310, pp. 5154-5157, 2008.

[2] R. H. Horng, C. C. Yang, J. Y. Wu, S. H. Huang, C. E. Lee, D. S. Wuu, "GaNbased light-emitting diodes with indium tin oxide texturing window layers using natural lithography," Appl. Phys. Lett., Vol. 86, 221101, 2005.

[3] H.W. Huang, C. C. Kao, J. T. Chu, H. C. Kuo, S. C. Wang, C. C. Yu, "Improved light-output and electrical performance of InGaN-based light-emitting diode by microroughening of the p-GaN surface," IEEE Phot. Tech. Lett., vol. 17, pp. 9383-9385, 2005.

[4] T. X. Lee, C. Y. Lin, S. H. Ma, C. C. Sun, “Analysis of position-dependent light extraction of GaN-based LEDs,” Optics Express, vol. 13, pp. 4175-4179, 2005.

[5] G. Hatakoshi, Y. Hattori, S. Saito, N. Shida, and S. Ninoue, "Device Simulator for Designing High-Efficiency Light-Emitting Diodes,’ Jpn. J. Appl. Phys., vol. 46(8B), pp. 5419-5425, 2007.

[6] Y. A. Chen, N. Y. Liang, L. H. Laih, W. C. Tsay, M. N. Chang, J. W. Hong, "Improvement of electroluminescence characteristics of porous silicon LED by using amorphous silicon layers," Elec. Lett., vol. 33, pp. 1489-1490, 1997.

[7] Y. A. Chen, N. Y. Liang, L. H. Laih, W. C. Tsay, M. N. Chang, J. W. Hong, "Improvement of current injection of porous silicon,” Jpn. J. Appl. Phys., vol. 36, 
pp. 1574-1577, 1997.

[8] H. W. Huang, C. C. Kao, H. C. Chu, S. C. Wang, C. C. Yu, "Improvement of InGaN-GaN Light-Emitting Diode Performance with a Nano-Roughened p-GaN Surface,” IEEE Phot. Tech. Lett., vol. 17, pp. 983-985, 2005.

[9] K. Bao, X. N. Kang, B. Zhang, T. Dai, C. Xiong, H. Ji, G. Y. Zhang, Y. Chen, "Improvement of Light Extraction from Patterned Polymer Encapsulated GaNBased Flip-Chip Light-Emitting Diodes by Imprinting," IEEE Phot. Tech. Lett., vol. 19, pp. 1840-1842, 2007.

[10] H. Y. Ryu, J. K. Kwang, Y. J. Lee, Y. H. Lee, "Enhancement of Light Extraction from Two-Dimensional Photonic Crystal Slab Structures,” IEEE J. on Sel. Top. in Quant. Elec., vol. 8, pp. 231-237, 2002.

[11] T. Kim, A. J. Danner, K. D. Choquette, "Enhancement in external quantum efficiency of blue light-emitting diode by photonic crystal surface grating," Elec. Lett., vol. 41, pp. 1138-1139, 2005.

[12] F. F. Ren, M. B. Yu, J. D. Ye, Q. Chen, G. Q. Lo, D. L. Kwong, “Enhanced Vertical Light Extraction from Ultrathin Amorphous Si-Si3N4 Multilayers with Photonic Crystal Patterns,” IEEE Phot. Tech. Lett., vol. 21, pp. 91-93, 2009.

[13] T. Dai, B. Zhang, Z.-S. Zhang, D. Liu, X. Wang, K. Bao, X.-N. Kang, J. Xu, D.-P. Yu, X. Zhu, "Surface Light Extraction Mapping from Two-Dimensional Array of 12-Fold Photonic Quasicrystal on Current Injected GaN-Based LEDs," Chinese Phys. Lett., vol. 24, pp. 979-982, 2007.

[14] R. J. Yan, Q. K. Wang, "Enhancement of Light Extraction Efficiency in OLED 
with Periodic Nano-Structure,” IEEE Intl. Conf. on Nano/Micro Eng. And Molecular Sys., Jan. 18-26, 2006.

[15] J. Q. Xi, H. Luo, A. J. Pasquale, J. K. Kim, E. F. Schubert, "Enhanced Light Extraction in GaInN Light-Emitting Diode with Pyramid Reflector,” IEEE Phot. Tech. Lett., vol. 18, pp. 2347-2349, 2006.

[16] S. Trieu, X. M. Jin, B. Zhang, T. Dai, K. Bao, X. N. Kang, G. Y. Zhang, "Light Extraction Improvement of GaN-based Light Emitting Diodes using Patterned Undoped GaN Bottom Reflection Gratings,” Proc. SPIE., Vol. 7216, pp. 72162Q72162Q-8, 2009.

[17] D. A. Steigerwald, J. C. Bhat, D. Collins, R. M. Fletcher, M. O. Holcomb, M. J. Ludowise, P. S. Martin, S. L. Rudaz, "Illumination with Solid State Lighting Technology,” IEEE J. on Sel. Top. in Quantum Elec., vol. 8, pp. 310-320, 2002.

[18] K. Bao, X. Kang, B. Zhang, T. Dai, Y. Sun, Q. Fu, G. Lian, G. Xiong, G. Zhang, Y. Chen, "Improvement of light extraction from GaN-based thin-film lightemitting diodes by patterning undoped GaN using modified laser lift-off," Appl. Phys. Lett., vol. 92, 141104, 2008.

[19] S. Kim, K. Lee, J. Kim, M. Kwon, S. Park, "Fabrication of photonic crystal structures on light emitting diodes by nanoimprint lithography," Nanotechnology, vol. 18(5), 055306 (5pp), 2007.

[20] T. Wriedt, “A Review of Elastic Light Scattering Theories," Part. Part. Syst. Charact., vol. 15, pp. 67-74, 1998.

[21] S. C. Hagness, D. Rafizadeh, S. T. Ho, A. Taflove, "FDTD Microcavity 
Simulations: Design and Experimental Realization of Waveguide-Coupled SingleMode Ring and Whispering-Gallery-Mode Disk Resonators," Journal of Lightwave Technology, vol. 15, pp. 2154-2165, 1997.

[22] R. Drezek, A. Dunn, R. Richards-Kortum, “A Pulsed Finite-Difference TimeDomain (FDTD) Method for Calculating Light Scattering from Biological Cells Over Broad Wavelength Ranges,” Optics Soc. Of Amer., Vol. 6, No. 7, pp. 147$157,2000$.

[23] J.M. Bourgeois, G.S. Smith, "A Fully Three-Dimensional Simulation of a Ground-Penetrating Radar: FDTD Theory Compared with Experiment," IEEE Trans. on Geo. and Rem. Sens., Vol. 34, No. 1, pp. 36-44, 1996.

[24] H.-R. Chuang, L.-C. Kuo, “3-D FDTD Design Analysis of a 2.4-GHz Polarization-Diversity Printed Dipole Antenna With Integrated Balun and Polarization-Switching Circuit for WLAN and Wireless Communication Applications," IEEE Transactions on Microwave Theory and Techniques, Vol. 51, No. 2, Part 1, pp. 374-381, 2003.

[25] K. Yee, "Numerical solution of initial boundary value problems involving Maxwell's equations in isotropic media," Antennas and Propagation, IEEE Transactions, vol. 14, pp. 302-307, 1966.

[26] Adams, S., Payne, J., Boppana, R., "Finite Difference Time Domain (FDTD) Simulations Using Graphics Processors”, HPCMP Users Group Conference, 2007.

[27] Liu, Z.M., Mohan, A.S., Aubrey, T.A., Belcher, W.R., “Techniques for 
Implementation of the FDTD Method on a CM-5 Parallel Computer", IEEE Ant. And Prop. Mag., 37, 5, 1995.

[28] Chen, Y., Simpson, T.L., Ho, T.Q., "Highly efficient technique for solving radiation and scattering problems", IEEE Proc., 139, 1, 1992.

[29] Kimura, H., Yoshida, N., "Three-dimensional full-wave analysis with nonlinearity and line characteristics of device by electromagnetic field analysis on time domain,” Elec. Comm. Jpn., 75, pp. 89-100, 1991.

[30] Rsoft Design Group, Inc., "RSoft Product Family," http://rsoftdesign.com/products.php?sub=Component+Design, web, accessed May, 92010.

[31] Jin, X., Zhang, B., Dai, T., Wei, W., Kang, X.-N., Zhang, G.-Y., Trieu,, S. and Wang, F., “Optimization of Top Polymer Gratings to Improve GaN LEDs Light Transmission”, Chinese Optics Lett. (Focus Issue Nano Photics), vol. 6, no. 10, pp. 788-790, 2008.

[32] RSoft Design Group, Inc., "FullWAVE 6.1: User Guide,” www.rsoftdesign.com, 2008.

[33] RSoft Design Group, Inc., "RSoft CAD Environment 8.1: User Guide", www.rsoftdesign.com, 2008.

[34] S. Nagahama, T. Yanamoto, M. Sano, T. Mukai, "Characteristics of InGaN laser diodes in the pure blue region,” Appl. Phys. Lett., vol. 79, pp. 1948-1951, 2001.

[35] A. Taflove, M.E. Brodwin, "Numerical solution of steady-state electromagnetic scattering problems using the time-dependent Maxwell's equations", IEEE Trans. 
Microwave Theory and Techniques, vol. 23, pp. 623-630, 1975.

[36] C. Xiong, B. Zhang, X.N. Kang, T. Dai, G.Y. Zhang, "Two-dimensional photonic quasicrystal on the surface of GaN-based light emitting diodes," Semicond. Sci. Technol., Vol. 25, 065006, 2010.

[37] E. Yablonovitch, "Inhibited Spontaneous Emission in Solid-State Physics and Electronics", Physical Review Letters, Vol. 58, No. 20, pp. 2059-2062, 1987.

[38] S. John, "Strong localization of photons in certain disordered dielectric superlattices", Physical Review Letters, Vol. 58, No. 23, pp. 2486-2489, 1987.

[39] S. J. Sheih, K. T. Tsen, D. K. Ferry, A. Botchkarev, B. Sverdlov, A. Salvador, and H. Morkoç, "Electron-phonon interactions in the wide band-gap semiconductor GaN,” Appl. Phys. Lett., Vol. 67, pp. 1757-1759, 1995.

[40] J. Goodman, "Introduction to Fourier Optics: Third Edition," Roberts \& Company Publishers, 2005.

[41] G.F. Brown, J.W. Ager III, W. Walukiewicz, J. Wu, "Finite element simulations of compositionally graded InGaN solar cells," Elsevier Solar Energy Materials \& Solar Cells, Vol. 94, pp. 478-483, 2010.

[42] M.H. Lu, J.C. Sturm, "Optimization of external coupling and light emission in organic light-emitting devices: modeling and experiment,” J. of Appl. Phys., Vol. 91, No. 2, pp. 595-604, 2002. 